\title{
Injectivity theorems with multiplier ideal sheaves for higher direct images under Kähler morphisms
}

\author{
Shin-ichi Matsumura
}

\begin{abstract}
In this paper, we establish injectivity theorems for higher direct image sheaves of canonical bundles twisted by pseudo-effective line bundles and multiplier ideal sheaves. As applications, we generalize Kollár's torsion-freeness and Grauert-Riemenschneider's vanishing theorem. Moreover, we obtain a relative vanishing theorem of KawamataViehweg-Nadel type and an extension theorem for holomorphic sections from fibers of morphisms. Our approach, based on transcendental methods, works for Kähler morphisms and singular Hermitian metrics with non-algebraic singularities.
\end{abstract}

\section{Introduction}

The injectivity theorem, which has been studied in the last decades, is a powerful tool in complex geometry and algebraic geometry, in particular birational geometry. After the pioneering work [Tan71] by Tankeev, Kollár [Kol86a] established the celebrated injectivity theorem by using Hodge theory. From the viewpoint of Hodge theory, we have already obtained useful generalizations (see, for example, [Amb03, Amb14, EV92, Fuj09, Fuj11, Fuj17, Fuj14, Kol86b]). Particularly, Theorem 1.1 below is one of the most important generalizations of Kollár's injectivity theorem for deformations of projective varieties. On the other hand, we can also approach Kollár's result from the analytic viewpoint (see, for example, [Eno93, Fuj12, Fuj13, FM21, Mat15b, Mat15a, Mat18a, Ohs05, Tak95, Tak97]). This paper contributes to the study of the injectivity theorem and its applications from the analytic viewpoint.

Theorem 1.1. Let $\pi: X \rightarrow \Delta$ be a surjective projective morphism from a smooth variety $X$ to a quasi-projective variety $\Delta$ and $F$ be a $\pi$-semi-ample line bundle on $X$.

Then, for a non-zero (holomorphic) section $s$ of $F^{m}$ (with $m \geqslant 0$ ), the multiplication map induced by the tensor product with $s$

$$
R^{q} \pi_{*}\left(K_{X} \otimes F\right) \stackrel{\otimes s}{\longrightarrow} R^{q} \pi_{*}\left(K_{X} \otimes F^{m+1}\right)
$$

is injective for every $q$. Here $K_{X}$ denotes the canonical bundle of $X$ and $R^{q} \pi_{*}(\bullet)$ denotes the $q$ th higher direct image sheaf.

Received 19 January 2018, accepted in final form 17 July 2021.

2020 Mathematics Subject Classification 32L20 (primary), 32L10, 14F18 (secondary).

Keywords: injectivity theorems, vanishing theorems, extension theorems, higher direct images, Kähler deformations, singular Hermitian metrics, multiplier ideal sheaves, Hodge theory, theory of harmonic integrals, $L^{2}$-methods, $\bar{\partial}$-equations.

This journal is (C) Foundation Compositio Mathematica 2022. This article is distributed with Open Access under the terms of the Creative Commons Attribution Non-Commercial License, which permits non-commercial reuse, distribution, and reproduction in any medium, provided that the original work is properly cited. For commercial re-use, please contact the Foundation Compositio Mathematica.

The author is supported by Grant-in-Aid for Young Scientists (A) $\sharp 17$ H04821, Grant-in-Aid for Scientific Research (B) $\sharp 21 \mathrm{H} 00976$, and Fostering Joint International Research (A) $\sharp 19$ KK0342 from JSPS. 


\section{INJECTIVITY THEOREMS FOR HIGHER DIRECT IMAGES}

In this paper, we consider a proper Kähler morphism $\pi: X \rightarrow \Delta$ from a complex manifold $X$ to an analytic space $\Delta$ and a (holomorphic) line bundle $F$ on $X$ equipped with a singular (Hermitian) metric $h$. Furthermore, we study the direct image sheaves $R^{q} \pi_{*}\left(K_{X} \otimes F \otimes \mathcal{I}(h)\right)$ of the canonical bundle $K_{X}$ on $X$ twisted by $F$ and the multiplier ideal sheaf $\mathcal{I}(h)$. This paper contains three main results: The first result is to establish two injectivity theorems formulated for Kähler morphisms and singular metrics with arbitrary singularities, which generalizes Theorem 1.1 to the complex-analytic setting (see Theorems 1.2 and 1.3). The second result, which is a direct application of our injectivity theorems, is to give Kollár's torsion-freeness and GrauertRiemenschneider's vanishing theorem for $R^{q} \pi_{*}\left(K_{X} \otimes F \otimes \mathcal{I}(h)\right.$ ) (see Corollary 1.5). The third result is to prove a relative vanishing theorem of Kawamata-Viehweg-Nadel type (see Theorem 1.7).

Enoki [Eno93] obtained the absolute case of Theorem 1.1 under the weaker assumption that $F$ is semi-positive (that is, it admits a smooth (Hermitian) metric with semi-positive curvature), as an application of the theory of harmonic integrals. Takegoshi [Tak95] proved the relative case of Enoki's result, which generalizes Theorem 1.1 from semi-ample line bundles to semi-positive line bundles. In this paper, we handle line bundles admitting a (possibly) singular metric with semi-positive curvature (namely, pseudo-effective line bundles). The study of pseudo-effective line bundles is an important subject; hence it is natural and of interest to pursue a further generalization of Theorem 1.1 and Takegoshi's result from semi-positive line bundles to pseudoeffective line bundles.

The following theorems, which are the main results of this paper, can be seen as generalizations of Theorem 1.1 and Takegoshi's result to pseudo-effective line bundles. Moreover, Theorems 1.2 and 1.3 include various injectivity theorems; see, for example, [Eno93, FM21, Fuj12, Fuj13, GM17, Kol86a, Mat14, Mat18a, Tak95, Tak97]. (See [CDM17, LRW19, Mat19, SZ21, ZZ19] and the references therein for recent developments.)

ThEOREM 1.2. Let $\pi: X \rightarrow \Delta$ be a surjective proper Kähler morphism from a complex manifold $X$ to an analytic space $\Delta$ and $(F, h)$ be a (possibly) singular Hermitian line bundle on $X$ with semi-positive curvature.

Then, for a non-zero (holomorphic) section $s$ of $F^{m}$ (with $m \geqslant 0$ ) satisfying $\sup _{K}|s|_{h^{m}}<\infty$ for every relatively compact set $K \Subset X$, the multiplication map induced by the tensor product with $s$

$$
R^{q} \pi_{*}\left(K_{X} \otimes F \otimes \mathcal{I}(h)\right) \stackrel{\otimes s}{\longrightarrow} R^{q} \pi_{*}\left(K_{X} \otimes F^{m+1} \otimes \mathcal{I}\left(h^{m+1}\right)\right)
$$

is injective for every $q$. Here $\mathcal{I}(\bullet)$ denotes the multiplier ideal sheaf of $\bullet$.

Theorem 1.3. Let $\pi: X \rightarrow \Delta$ be a surjective proper Kähler morphism from a complex manifold $X$ to an analytic space $\Delta$. Let $(F, h)$ be a (possibly) singular Hermitian line bundle on $X$ and $\left(M, h_{M}\right)$ be a smooth Hermitian line bundle on $X$. Assume

$$
\sqrt{-1} \Theta_{h_{M}}(M) \geqslant 0 \text { and } \sqrt{-1}\left(\Theta_{h}(F)-b \Theta_{h_{M}}(M)\right) \geqslant 0
$$

for some $b>0$.

Then, for a non-zero (holomorphic) section $s$ of $M$, the multiplication map induced by the tensor product with $s$

$$
R^{q} \pi_{*}\left(K_{X} \otimes F \otimes \mathcal{I}(h)\right) \stackrel{\otimes s}{\longrightarrow} R^{q} \pi_{*}\left(K_{X} \otimes F \otimes \mathcal{I}(h) \otimes M\right)
$$

is injective for every $q$. 


\section{S. Matsumura}

Remark 1.4. (1) A proper morphism $\pi: X \rightarrow \Delta$ is called a Kähler morphism in this paper if, for every point $t \in \Delta$, there exists an open neighborhood $\Delta^{\prime}$ of $t$ such that $\pi^{-1}\left(\Delta^{\prime}\right)$ is a Kähler manifold.

(2) The case $m=0$ in Theorem 1.2 coincides with the case where $\left(M, h_{M}\right)$ is trivial in Theorem 1.3. This case is important for applications.

(3) The assumption in Theorem 1.2 on the local sup-norm is a reasonable condition to make the multiplication map well defined and is always satisfied in the case $m=0$.

The absolute case of the above results has been obtained in [Mat18a, FM21], by combining the theory of harmonic integrals with the $L^{2}$-method for the $\bar{\partial}$-equation (see [GM17, FM21] for applications). The proof of the main results is based on transcendental methods developed in [Mat18a, FM21, Tak95]. One of the advantages of our methods is that we can prove the main results for Kähler morphisms (not only projective morphisms) and for singular metrics with nonalgebraic singularities. Under the regularity of singular metrics, a theorem similar to Theorem 1.3 was given in [Fuj13]. Nevertheless, we are sometimes faced with the case of handling a singular metric $h$ obtained from a suitable limit of metrics $\left\{h_{m}\right\}_{m=1}^{\infty}$; furthermore, it is quite hard to investigate the regularity of the limit $h$ even when $h_{m}$ has algebraic singularities. Therefore, it is worth formulating Theorems 1.2 and 1.3 for singular metrics with arbitrary singularities.

As a direct corollary, we generalize Kollár's torsion-freeness [Kol86a] and Grauert-Riemenschneider's vanishing theorem [GR70] for the higher direct images $R^{q} \pi_{*}\left(K_{X} \otimes F \otimes \mathcal{I}(h)\right)$.

Corollary 1.5 (Kollár's torsion-freeness, Grauert-Riemenschneider's vanishing theorem). Let $\pi: X \rightarrow \Delta$ be a surjective proper Kähler morphism from a complex manifold $X$ to an analytic space $\Delta$ and $(F, h)$ be a (possibly) singular Hermitian line bundle on $X$ with semi-positive curvature.

Then, the higher direct image sheaf $R^{q} \pi_{*}\left(K_{X} \otimes F \otimes \mathcal{I}(h)\right)$ is torsion-free for every $q$. Moreover, we obtain

$$
R^{q} \pi_{*}\left(K_{X} \otimes F \otimes \mathcal{I}(h)\right)=0 \quad \text { for every } q>\operatorname{dim} X-\operatorname{dim} \Delta .
$$

As a further application, we obtain a vanishing theorem of Kawamata-Viehweg-Nadel type [Kaw82, Vie82, Nad90] for the higher direct images $R^{q} \pi_{*}\left(K_{X} \otimes F \otimes \mathcal{I}(h)\right)$ (see Theorem 1.7). The proof of Theorem 1.7 requires the lower semi-continuity of the numerical Kodaira dimension of singular Hermitian line bundles, which is of independent interest.

Proposition 1.6 (Quasi-lower semi-continuity of the numerical Kodaira dimension). Let $\pi: X \rightarrow$ $\Delta$ be a surjective projective morphism from a complex manifold $X$ to an analytic space $\Delta$ and $(F, h)$ be a (possibly) singular Hermitian line bundle on $X$ with semi-positive curvature. Assume that $\pi$ is smooth at a point $t_{0} \in \Delta$.

Then, there exist an open neighborhood $B$ of $t_{0}$ and a dense subset $Q \subset B$ such that

$$
\text { for every } t \in Q \text {, the inequality } \operatorname{nd}\left(\left.F\right|_{X_{t}},\left.h\right|_{X_{t}}\right) \geqslant \operatorname{nd}\left(\left.F\right|_{X_{t_{0}}},\left.h\right|_{X_{t_{0}}}\right) \text { holds. }
$$

Here $\left(\left.F\right|_{X_{t}},\left.h\right|_{X_{t}}\right)$ denotes the singular Hermitian line bundle restricted to the fiber $X_{t}:=\pi^{-1}(t)$ and $\operatorname{nd}\left(\left.F\right|_{X_{t}},\left.h\right|_{X_{t}}\right)$ denotes its numerical Kodaira dimension.

See Definition 4.1 for the precise definition of the numerical Kodaira dimension.

By combining the vanishing theorem [Cao14] and the strong openness theorem [GZ15] with the proof of Proposition 1.6, we obtain a relative vanishing theorem of Kawamata-ViehwegNadel type. Note that Proposition 1.6 and Theorem 1.7 have been further generalized to Kähler morphisms by the same strategy as that in this paper (see [Mat18b, Theorems 1.1 and 1.2]). 


\section{INJECTIVITY THEOREMS FOR HIGHER DIRECT IMAGES}

THEOREM 1.7 (Relative vanishing theorem of Kawamata-Viehweg-Nadel type). Let $\pi: X \rightarrow \Delta$ be a surjective projective morphism from a complex manifold $X$ to an analytic space $\Delta$ and $(F, h)$ be a (possibly) singular Hermitian line bundle on $X$ with semi-positive curvature.

Then, we have

$$
R^{q} \pi_{*}\left(K_{X} \otimes F \otimes \mathcal{I}(h)\right)=0 \quad \text { for every } q>f-\max _{\substack{\pi \text { smooth } \\ \text { at } t \in \Delta}} \operatorname{nd}\left(\left.F\right|_{X_{t}},\left.h\right|_{X_{t}}\right),
$$

where $f$ is the relative dimension of $\pi: X \rightarrow \Delta$. In particular, if $\left(\left.F\right|_{X_{t}},\left.h\right|_{X_{t}}\right)$ is big for some point $t$ in the smooth locus of $\pi$, then we have

$$
R^{q} \pi_{*}\left(K_{X} \otimes F \otimes \mathcal{I}(h)\right)=0 \quad \text { for every } q>0 .
$$

Moreover, we obtain an extension theorem (see Corollary 1.9), motivated by the invariance of plurigenera and the divisorially log-terminal (dlt) extension conjecture in the minimal model program (see [Lev83, Siu98, Siu02, Tak07, Pău07] for the invariance of plurigenera, [DHP13, FG14, GM17] for the dlt extension conjecture, and the references therein). When $\pi: X \rightarrow \Delta$ is a smooth projective morphism, the following problem corresponds to the so-called invariance of plurigenera proved by Siu [Siu98, Siu02] (see also [Pău07]).

Problem 1.8. Let $\pi: X \rightarrow \Delta$ be a surjective proper Kähler morphism from a complex manifold $X$ to an open disk $\Delta \subset \mathbb{C}$. Assume that $K_{X}$ is $\pi$-nef and the central fiber $X_{0}:=\pi^{-1}(0)$ is simple normal crossing. Then, can we extend a section $u \in H^{0}\left(X_{0}, \mathcal{O}_{X_{0}}\left(K_{X}^{m}\right)\right)$ to a section in $H^{0}\left(X, \mathcal{O}_{X}\left(K_{X}^{m}\right)\right)$ (by replacing $\Delta$ with a smaller disk if necessary)?

When $\pi: X \rightarrow \Delta$ is a projective morphism, Takayama [Tak07] showed that every section of the pluri-canonical bundle on each component of $X_{0}$ can be extended. When $\pi: X \rightarrow \Delta$ is a non-projective smooth morphism, we have some partial results in [Lev83, CP20]. The following result, which can be seen as a relative version of [GM17, Theorem 1.4], partially solves the above problem when $K_{X}$ admits a singular metric with mild singularities.

Corollary 1.9. In the same situation as in Problem 1.8, let $(F, h)$ be a singular Hermitian line bundle on $X$ with semi-positive curvature. Then, every section in $H^{0}\left(X_{0}, \mathcal{O}_{X_{0}}\left(K_{X} \otimes F\right)\right)$ that comes from $H^{0}\left(X_{0}, \mathcal{O}_{X_{0}}\left(K_{X} \otimes F\right) \otimes \mathcal{I}(h)\right)$ can be extended to a section in $H^{0}\left(X, \mathcal{O}_{X}\left(K_{X} \otimes\right.\right.$ $F) \otimes \mathcal{I}(h)$ ) by replacing $\Delta$ with a smaller disk. In particular, if $K_{X}$ admits a singular metric $h$ whose curvature is semi-positive and whose Lelong number is zero at every point in $X_{0}$, then Problem 1.8 is affirmatively solved.

In the rest of this section, we briefly explain the proof of Theorems 1.2, comparing our case to the absolute case proved in [Mat18a]. The proof of Theorem 1.3 is essentially the same as that of Theorem 1.2. For the proof of the main results, we generalize the methods in [Tak95] and [Fuj13] and combine them with techniques in [FM21] and [Mat18a].

In Step 1, we approximate a given singular metric $h$ by singular metrics $\left\{h_{\varepsilon}\right\}_{\varepsilon>0}$ that are smooth on a Zariski open set $Y_{\varepsilon}$, which enables us to apply the theory of harmonic integrals on $Y_{\varepsilon}$. Note that we cannot directly apply the theory of harmonic integrals since $h$ may have non-algebraic singularities. The subvariety $Y_{\varepsilon}$ does not depend on $\varepsilon$ in [Mat18a], but $Y_{\varepsilon}$ essentially depends on $\varepsilon$ in our case. To overcome this difficulty, we construct a complete Kähler form $\omega_{\varepsilon, \delta}$ on $Y_{\varepsilon}$ such that $\omega_{\varepsilon, \delta}$ converges to a Kähler metric $\omega$ on $X$ as $\delta \rightarrow 0$. By the standard de RhamWeil isomorphism, we can represent a given cohomology class $\{u\}$ by an $F$-valued differential form $u$. The $F$-valued form $u$ is locally $L^{2}$-integrable, but $u$ may not be globally $L^{2}$-integrable 


\section{S. Matsumura}

on $X$ due to the non-compactness of $X$. For this reason, in Step 2, we construct a new metric $H_{\varepsilon}$ on $F$ by suitably choosing an exhaustive plurisubharmonic function on $X$, so that the same cohomology class $\{u\}$ can be represented by harmonic $L^{2}$-forms $u_{\varepsilon, \delta}$ with respect to $H_{\varepsilon}$ and $\omega_{\varepsilon, \delta}$. In Step 3, we reduce the proof to showing that the $L^{2}$-norm $\left\|s u_{\varepsilon, \delta}\right\|_{X_{c}, H_{\varepsilon}, \omega_{\varepsilon, \delta}}$ on a relatively compact set $X_{c} \Subset X$ converges to zero as $\varepsilon \rightarrow 0$ and $\delta \rightarrow 0$. For this step, we need that the quotient map from the local $L^{2}$-space to the $\bar{\partial}$-cohomology group is a compact operator (see Proposition 2.19). In Step 4, using the Cech complex and the de Rham-Weil isomorphism, we construct a solution $v_{\varepsilon, \delta}$ of the $\bar{\partial}$-equation $\bar{\partial} v_{\varepsilon, \delta}=s u_{\varepsilon, \delta}$ such that the $L^{2}$-norm $\left\|v_{\varepsilon, \delta}\right\|_{X_{c}, H_{\varepsilon}, \omega_{\varepsilon, \delta}}$ on $X_{c}$ is uniformly bounded. We then obtain that

$$
\begin{aligned}
\left\|s u_{\varepsilon, \delta}\right\|_{X_{c}, H_{\varepsilon}, \omega_{\varepsilon, \delta}}^{2} & =\left\langle\left\langle s u_{\varepsilon, \delta}, \bar{\partial} v_{\varepsilon, \delta}\right\rangle_{X_{c}, H_{\varepsilon}, \omega_{\varepsilon, \delta}}\right. \\
& =\left\langle\left\langle\bar{\partial}^{*} s u_{\varepsilon, \delta}, v_{\varepsilon, \delta}\right\rangle_{X_{c}, H_{\varepsilon}, \omega_{\varepsilon, \delta}}+\left(\left((d \Phi)^{*} s u_{\varepsilon, \delta}, v_{\varepsilon, \delta}\right)\right)_{\partial X_{c}, H_{\varepsilon}, \omega_{\varepsilon, \delta}}\right.
\end{aligned}
$$

for almost all $X_{c} \Subset X$, by generalizing the formula in [FK72] (see Proposition 2.5). Here •* denotes the adjoint operator of $\bullet$ and $(\bullet, \bullet))_{\partial X_{c}}$ denotes the norm on the boundary $\partial X_{c}$. Note that the norm on the boundary $\partial X_{c}$ appears due to the non-compactness of $X$. In Step 5 , we show that the norm of $\bar{\partial}^{*} s u_{\varepsilon, \delta}$ and $(d \Phi)^{*} s u_{\varepsilon, \delta}$ converges to zero by applying Ohsawa-Takegoshi's twisted Bochner-Kodaira-Nakano identity.

The remainder of this paper is organized as follows: In Section 2, we summarize the results needed in this paper. Moreover, in that section, we give a generalization of [FK72, (1.3.2) Proposition] and recall the fundamental facts on the construction of the de Rham-Weil isomorphism in our situation. In Section 3, we prove Theorems 1.2 and 1.3. In Section 4, we prove Corollary 1.5, Proposition 1.6, Theorem 1.7, and Corollary 1.9.

\section{Preliminaries}

In this section, we summarize the results used in this paper. Throughout this section, let $X$ be a complex manifold of dimension $n$ and $F$ be a (holomorphic) line bundle on $X$.

\section{1 $L^{2}$-spaces of differential forms}

In this subsection, we recall $L^{2}$-spaces of $F$-valued differential forms and operators defined on them. Let $\omega$ be a positive $(1,1)$-form on $X$ and $h$ be a smooth (Hermitian) metric on $F$.

For $F$-valued $(p, q)$-forms $u$ and $v$, the (global) inner product $\langle u, v\rangle_{h, \omega}$ is defined by

$$
\langle u, v\rangle_{h, \omega}:=\int_{X}\langle u, v\rangle_{h, \omega} d V_{\omega}
$$

where $d V_{\omega}$ is the volume form defined by $d V_{\omega}:=\omega^{n} / n$ ! and $\langle u, v\rangle_{h, \omega}$ is the point-wise inner product with respect to $h$ and $\omega$. The $L^{2}$-space of $F$-valued $(p, q)$-forms with respect to $h$ and $\omega$ is defined by

$$
L_{(2)}^{p, q}(X, F)_{h, \omega}:=\left\{u \mid u \text { is an } F \text {-valued }(p, q) \text {-form with }\|u\|_{h, \omega}<\infty\right\} .
$$

The Chern connection $D=D_{(F, h)}$ on $F$ is canonically determined by the holomorphic structure and the smooth metric $h$ on $F$, which can be written as $D=D_{h}^{\prime}+D_{h}^{\prime \prime}$ with the $(1,0)$ connection $D_{h}^{\prime}$ and the $(0,1)$-connection $D_{h}^{\prime \prime}$. Note that $D_{h}^{\prime \prime}$ agrees with the $\bar{\partial}$-operator. The connections $\bar{\partial}$ and $D_{h}^{\prime}$ (strictly speaking, their maximal extension) can be seen as densely defined 


\section{INJECTIVITY THEOREMS FOR HIGHER DIRECT IMAGES}

closed operators on $L_{(2)}^{p, q}(X, F)_{h, \omega}$ with domain

$$
\operatorname{Dom} \bar{\partial}:=\left\{u \in L_{(2)}^{p, q}(X, F)_{h, \omega} \mid \bar{\partial} u \in L_{(2)}^{p, q+1}(X, F)_{h, \omega}\right\} .
$$

These operators depend on $h$ and $\omega$ in the sense that their domain and range depend on them, but we often omit the subscript (for example, we abbreviate $\bar{\partial}_{h, \omega}$ to $\bar{\partial}$ ).

We consider the Hodge star operator $*$ with respect to $\omega$

$$
*=*_{\omega}: C_{\infty}^{p, q}(X, F) \rightarrow C_{\infty}^{n-q, n-p}(X, F),
$$

where $C_{\infty}^{p, q}(X, F)$ is the set of smooth $F$-valued $(p, q)$-forms on $X$. By definition, we have $\langle u, v\rangle_{h, \omega} d V_{\omega}=u \wedge H \overline{* v}$, where $H$ is a local function representing $h$. In this paper, the notation $A^{*}$ denotes the formal adjoint of an operator $A$. For example, the symbols $D_{h, \omega}^{\prime *}$ and $\bar{\partial}_{h, \omega}^{*}$ denote the formal adjoint operators of $D_{h, \omega}^{\prime}$ and $\bar{\partial}$, respectively. Note that

$$
D_{h, \omega}^{\prime *}=-* \bar{\partial} * \quad \text { and } \quad \bar{\partial}_{h, \omega}^{*}=-* D_{h, \omega}^{\prime} * .
$$

Furthermore, for a differential form $\theta$, the symbol $\theta^{*}$ denotes the adjoint operator with respect to the point-wise inner product. When $\theta$ is of type $(s, t)$ and $\theta^{*}$ acts on $C_{\infty}^{p, q}(X, F)$, we have

$$
\theta^{*}=(-1)^{(p+q)(s+t+1)} * \bar{\theta} * \text {. }
$$

For operators $A$ and $B$ with pure degree, the graded bracket $[\bullet, \bullet]$ is defined by

$$
[A, B]:=A B-(-1)^{\operatorname{deg} A \operatorname{deg} B} B A .
$$

In the proof of the main results, we often use the following lemmas, which are obtained from simple computations (see, for example, [Tak95] for Lemma 2.1).

LEMma 2.1. If $\omega$ is a Kähler form, then we have the following identities:

- $\theta^{*}=\sqrt{-1}\left[\bar{\theta}, \Lambda_{\omega}\right]$ for a $(1,0)$-form $\theta$,

- $\eta^{*}=-\sqrt{-1}\left[\bar{\eta}, \Lambda_{\omega}\right]$ for a $(0,1)$-form $\eta$,

- $\left[\bar{\partial},(\bar{\partial} \Phi)^{*}\right]+\left[D_{h, \omega}^{\prime *}, \partial \Phi\right]=\left[\sqrt{-1} \partial \bar{\partial} \Phi, \Lambda_{\omega}\right]$ for a smooth function $\Phi$.

Here $\Lambda_{\omega}$ denotes the adjoint operator defined by $\Lambda_{\omega}:=\omega^{*}$.

Lemma 2.2. Let $\widetilde{\omega}$ and $\omega$ be positive $(1,1)$-forms such that $\widetilde{\omega} \geqslant \omega$.

- There exists a $C>0$ such that $\left|\theta^{*} u\right|_{\omega} \leqslant C|\theta|_{\omega}|u|_{\omega}$ for differential forms $\theta$ and $u$.

- The inequality $|\theta|_{\widetilde{\omega}} \leqslant|\theta|_{\omega}$ holds for a differential form $\theta$.

- The inequality $|\theta|_{\widetilde{\omega}} d V_{\widetilde{\omega}} \leqslant|\theta|_{\omega} d V_{\omega}$ holds for an $(n, q)$-form $\theta$.

- The equality $|\theta|_{\widetilde{\omega}} d V_{\widetilde{\omega}}=|\theta|_{\omega} d V_{\omega}$ holds for an $(n, 0)$-form $\theta$.

Proof. For a given point $x \in X$, we choose a local coordinate $\left(z_{1}, z_{2}, \ldots, z_{n}\right)$ such that

$$
\widetilde{\omega}=\frac{\sqrt{-1}}{2} \sum_{j=1}^{n} \lambda_{j} d z_{j} \wedge d \overline{z_{j}} \quad \text { and } \quad \omega=\frac{\sqrt{-1}}{2} \sum_{j=1}^{n} d z_{j} \wedge d \overline{z_{j}} \quad \text { at } x .
$$

When the differential forms $\theta$ and $u$ are written as

$$
\theta=\sum_{I, J} \theta_{I, J} d z_{I} \wedge d \bar{z}_{J} \quad \text { and } \quad u=\sum_{K, L} u_{K, L} d z_{K} \wedge d \bar{z}_{L}
$$

in terms of this coordinate, we have

$$
|\theta|_{\omega}^{2}=\sum_{I, J}\left|\theta_{I, J}\right|^{2} \quad \text { and } \quad|\theta|_{\widetilde{\omega}}^{2}=\sum_{I, J}\left|\theta_{I, J}\right|^{2} \frac{1}{\prod_{(i, j) \in(I, J)} \lambda_{i} \lambda_{j}}
$$




\section{S. Matsumura}

at $x$, where $I, J, K, L$ are ordered multi-indices. The second claim follows from $\lambda_{i} \geqslant 1$. Furthermore, the third claim and the fourth claim can be easily obtained from the above equalities. From $\left|\theta_{I, J}\right| \leqslant|\theta|_{\omega}$ and $\left|u_{K, L}\right| \leqslant|u|_{\omega}$, we have

$$
\begin{aligned}
|\theta \wedge u|_{\omega} & =\left|\sum_{I, J, K, L} \theta_{I, J} u_{K, L} d z_{I} \wedge d \bar{z}_{J} \wedge d z_{K} \wedge d \bar{z}_{L}\right|_{\omega} \\
& \leqslant \sum_{I, J, K, L}\left|\theta_{I, J}\right|\left|u_{K, L}\right| \leqslant \sum_{I, J, K, L}|\theta|_{\omega}|u|_{\omega}=C_{1}|\theta|_{\omega}|u|_{\omega}
\end{aligned}
$$

where $C_{1}$ is a positive constant depending only on the degree of the differential forms. Since the Hodge star operator $*$ preserves the point-wise norm, we have

$$
\left|\theta^{*} u\right|_{\omega}=|* \bar{\theta} * u|_{\omega}=|\bar{\theta} * u|_{\omega} \leqslant C_{2}|\bar{\theta}|_{\omega}|* u|_{\omega}=C_{2}|\theta|_{\omega}|u|_{\omega}
$$

for some constant $C_{2}>0$.

\subsection{Ohsawa-Takegoshi's twisted Bochner-Kodaira-Nakano identity}

The following proposition is obtained from the twisted Bochner-Kodaira-Nakano identity (cf. [DF83, DX84, OT87, Ohs95]), which plays an important role in Step 5. See, for example, [Tak95, Theorem 2.2] for the precise proof.

Proposition 2.3 (Twisted Bochner-Kodaira-Nakano identity). Let $\omega$ be a complete Kähler form on $X$ such that $\sqrt{-1} \Theta_{h}(F) \geqslant-C_{1} \omega$ for some constant $C_{1}$. Furthermore, let $\Phi$ be a bounded smooth function on $X$ such that $\sup _{X}|d \Phi|_{\omega}<\infty$ and $\sqrt{-1} \partial \bar{\partial} \Phi \geqslant-C_{2} \omega$ for some constant $C_{2}$.

Then, for every $u \in \operatorname{Dom} \bar{\partial}_{h, \omega}^{*} \cap \operatorname{Dom} \bar{\partial} \subset L_{(2)}^{n, q}(X, F)_{h, \omega}$, we have

$$
\begin{aligned}
& \|\sqrt{\eta}(\bar{\partial}+\bar{\partial} \Phi) u\|_{h, \omega}^{2}+\left\|\sqrt{\eta} \bar{\partial}_{h, \omega}^{*} u\right\|_{h, \omega}^{2} \\
& \quad=\left\|\sqrt{\eta}\left(D_{h, \omega}^{\prime *}-(\partial \Phi)^{*}\right) u\right\|_{h, \omega}^{2}+\left\langle\eta \sqrt{-1}\left(\Theta_{h}(F)+\partial \bar{\partial} \Phi\right) \Lambda_{\omega} u, u\right\rangle_{h, \omega},
\end{aligned}
$$

where $\eta$ is the function defined by $\eta:=e^{\Phi}$.

The case $\Phi \equiv 0$ corresponds to the non-twisted version. In the proof of Proposition 2.5 (and that of Proposition 2.3), we use the following lemma due to Andreotti-Vesentini (see [AV65, Ves67] and [Dem82, Lemme 4.3]).

Lemma 2.4 (Density lemma). Let $\omega$ be a complete positive $(1,1)$-form on $X$.

- There exists a sequence of cut-off functions $\left\{\theta_{k}\right\}_{k=1}^{\infty}$ on $X$ such that

$$
\operatorname{Supp} \theta_{k} \Subset X, \quad\left|d \theta_{k}\right|_{\omega} \leqslant 1, \quad \text { and } \theta_{k} \rightarrow 1 \text { as } k \rightarrow \infty \text {. }
$$

- The set of smooth F-valued $(p, q)$-forms with compact support is dense in Dom $\bar{\partial}_{h, \omega}^{*}$, Dom $\bar{\partial}$, and Dom $\bar{\partial}_{h, \omega}^{*} \cap$ Dom $\bar{\partial}$, respectively, with respect to the following graph norms:

$$
\|u\|_{h, \omega}+\left\|\bar{\partial}_{h, \omega}^{*} u\right\|_{h, \omega}, \quad\|u\|_{h, \omega}+\|\bar{\partial} u\|_{h, \omega}, \quad \text { and } \quad\|u\|_{h, \omega}+\left\|\bar{\partial}_{h, \omega}^{*} u\right\|_{h, \omega}+\|\bar{\partial} u\|_{h, \omega} .
$$

\subsection{Adjoint operators on domains with boundaries}

Let $\Phi$ be a smooth function on $X$. In this subsection, we consider the level set $X_{c}$ defined by $X_{c}:=\{x \in X \mid \Phi(x)<c\}$ such that $X_{c} \Subset X$ and $d \Phi \neq 0$ on the boundary $\partial X_{c}$ of $X_{c}$. The inner product on the boundary $\partial X_{c}$ is defined to be

$$
((u, v))_{\partial X_{c}, h, \omega}:=\int_{\partial X_{c}}\langle u, v\rangle_{h, \omega} d S_{\omega}
$$




\section{INJECTIVITY THEOREMS FOR HIGHER DIRECT IMAGES}

for $F$-valued $(p, q)$-forms $u$ and $v$ that are smooth on a neighborhood of $\partial X_{c}$. Here $d S_{\omega}$ is the volume form on $\partial X_{c}$ defined by $d S_{\omega}:=* d \Phi /|d \Phi|_{\omega}^{2}$. Note that we have $d V_{\omega}=d \Phi \wedge d S_{\omega}$ by definition. Stoke's theorem yields

$$
\langle\bar{\partial} u, v\rangle_{X_{c}, h, \omega}=\left\langle u, \bar{\partial}_{h, \omega}^{*} v\right\rangle_{X_{c}, h, \omega}+\left(\left(u,(\bar{\partial} \Phi)^{*} v\right)_{\partial X_{c}, h, \omega}\right.
$$

for a smooth $F$-valued ( $p, q-1)$-form $u$ and a $(p, q)$-form $v$ on $X$ (see [FK72, (1.3.2) Proposition]).

For our purposes, we need to generalize the above formula to a Zariski open set $Y \subset X$ equipped with a complete positive $(1,1)$-form $\widetilde{\omega}$. In the following proposition, we consider the Hodge star operator $*:=*_{\widetilde{\omega}}$, the volume form $d S_{\widetilde{\omega}}:=*_{\widetilde{\omega}} d \Phi /|d \Phi|_{\widetilde{\omega}}^{2}$, the inner product

$$
((\bar{\partial} u, v))_{\partial X_{c}, h, \widetilde{\omega}}:=\int_{\partial X_{c} \cap Y}\langle u, v\rangle_{h, \widetilde{\omega}} d S_{\widetilde{\omega}},
$$

and so on with respect to $\widetilde{\omega}($ not $\omega)$.

Proposition 2.5. Let $\widetilde{\omega}$ be a complete positive $(1,1)$-form on a Zariski open set $Y$ of a complex manifold $X$. Let $u$ and $v$, respectively, be a smooth $F$-valued $(p, q-1)$-form and a $F$-valued $(p, q)$-form on $Y$ with the finite $L^{2}$-norms $\|u\|_{h, \widetilde{\omega}},\|v\|_{h, \widetilde{\omega}},\|\bar{\partial} u\|_{h, \widetilde{\omega}},\left\|\bar{\partial}_{h, \widetilde{\omega}}^{*} v\right\|_{h, \widetilde{\omega}}$. Consider a smooth function $\Phi$ on $X$ and the level set $X_{d}$ defined by $X_{d}:=\{x \in X \mid \Phi(x)<d\}$ for $d \in \mathbb{R}$. If $X_{c} \Subset X$ and $d \Phi \neq 0$ on the boundary $\partial X_{c}$ of $X_{c}$ for some $c \in \mathbb{R}$, then there exists a sufficiently small number $a>0$ with the following properties:

(a) $d \Phi \neq 0$ on $\partial X_{d}$ for every $d \in(c-a, c+a)$,

(b) $\langle\bar{\partial} u, v\rangle_{X_{d}, h, \widetilde{\omega}}=\left\langle\left\langle u, \bar{\partial}_{h, \widetilde{\omega}}^{*} v\right\rangle_{X_{d}, h, \widetilde{\omega}}+\left(\left(u,(\bar{\partial} \Phi)^{*} v\right)\right)_{\partial X_{d}, h, \widetilde{\omega}}\right.$ for almost all $d \in(c-a, c+a)$.

Remark 2.6. (1) In the case $Y=X$, the equality in property (b) above holds for arbitrary $d \in(c-a, c+a)$ (see [FK72, (1.3.2) Proposition]).

(2) From the proof, we see that the real number $a$ depends only on $\Phi$, not on $u, v$, and $\widetilde{\omega}$.

(3) In the proof of the main results, we apply Proposition 2.5 to countably many differential forms. The subset $I$ defined by

$$
I:=\{d \in(c-a, c+a) \mid \text { the equality in property (b) of Proposition } 2.5 \text { does NOT hold for } d\}
$$

depends on $u, v, \widetilde{\omega}$. The Lebesgue measure of $I$ is zero by the proposition, and a countable union of subsets of Lebesgue measure zero also has Lebesgue measure zero. Hence, for given countably many differential forms, there exists a uniform $I$ of Lebesgue measure zero such that the equality in property (b) of Proposition 2.5 holds for $d \notin I$.

Proof of Proposition 2.5. For simplicity, we consider only the case where $(F, h)$ is trivial. For a sufficiently small $a>0$, we have $d \Phi \neq 0$ on $\partial X_{d}$ for every $d \in(c-a, c+a)$ by the assumption $d \Phi \neq 0$ on $\partial X_{c}$. For the proof of property (b), we take a sequence of cut-off functions $\left\{\theta_{k}\right\}_{k=1}^{\infty}$ on $Y$ such that $\operatorname{Supp} \theta_{k} \Subset Y$ and $\theta_{k} \rightarrow 1$ as $k \rightarrow \infty$. Since $\widetilde{\omega}$ is complete on $Y$, we can add the property $\left|d \theta_{k}\right|_{\widetilde{\omega}} \leqslant 1$ (see Lemma 2.4). Then, Stoke's theorem implies that

$$
\left\langle\bar{\partial}\left(\theta_{k} u\right), v\right\rangle_{X_{d}, \widetilde{\omega}}=\left\langle\theta_{k} u, \bar{\partial}_{\widetilde{\omega}}^{*} v\right\rangle_{X_{d}, \widetilde{\omega}}+\left(\left(\theta_{k} u,(\bar{\partial} \Phi)^{*} v\right)\right)_{\partial X_{d}, \widetilde{\omega}} .
$$

Note that all integrals and adjoint operators are computed with respect to $\widetilde{\omega}$ (not $\omega$ ). There is no difficulty in proving equality (2.1) since all integrands that appear in equality (2.1) are zero on a neighborhood of the subvariety $X \backslash Y$. Indeed, from Stoke's theorem and

$$
\left(d \theta_{k} u\right) \wedge \overline{* v}=-\theta_{k} u \wedge(\overline{* * d * v})+d\left(\theta_{k} u \wedge \overline{* v}\right),
$$




\section{S. Matsumura}

we have

$$
\left\langle\bar{\partial}\left(\theta_{k} u\right), v\right\rangle_{X_{d}, \widetilde{\omega}}=\left\langle\theta_{k} u, \bar{\partial}_{\widetilde{\omega}}^{*} v\right\rangle_{X_{d}, \widetilde{\omega}}+\int_{\partial X_{d}} \theta_{k} u \wedge \overline{* v} .
$$

Furthermore, we have

$$
\{u \wedge \overline{* v}\} \wedge d \Phi=-u \wedge \overline{* * d \Phi \wedge * v}=-\left\langle u,(d \Phi)^{*} v\right\rangle d V_{\widetilde{\omega}}=\left\{\left\langle u,(d \Phi)^{*} v\right\rangle d S_{\widetilde{\omega}}\right\} \wedge d \Phi .
$$

It follows that $d \Phi$ is non-zero in the normal direction of $\partial X_{d}$ from $\left.d \Phi\right|_{\partial X_{d}}=0$ and $d \Phi \neq 0$ on $\partial X_{d}$. Therefore, we can conclude that

$$
\int_{\partial X_{d}} u \wedge \overline{* v}=\int_{\partial X_{d}}\left\langle u,(d \Phi)^{*} v\right\rangle d S_{\widetilde{\omega}}
$$

Hence we obtain equality (2.1).

Now, we observe the limit of each term. The bounded Lebesgue convergence theorem implies that $\theta_{k} u$ converges to $u$ as $k \rightarrow \infty$ in the $L^{2}$-topology with respect to $\widetilde{\omega}$. Here we used the assumption $\|u\|_{\widetilde{\omega}}<\infty$. On the other hand, since $\bar{\partial}\left(\theta_{k} u\right)=\bar{\partial} \theta_{k} \wedge u+\theta_{k} \bar{\partial} u$ and $\left|d \theta_{k}\right|_{\widetilde{\omega}} \leqslant 1$ and $d \theta_{k} \rightarrow 0$ in the point-wise sense, we can easily see that $\bar{\partial} \theta_{k} \wedge u \rightarrow 0$ and $\theta_{k} \bar{\partial} u \rightarrow \bar{\partial} u$ in the $L^{2}$-topology, by using the bounded Lebesgue convergence theorem again. Here we used the assumption $\|\bar{\partial} u\|_{\widetilde{\omega}}<\infty$. From the above argument, the left-hand side (respectively, the first term of the right-hand side) of equality (2.1) converges to $\langle\bar{\partial} u, v\rangle_{X_{d}, \widetilde{\omega}}$ (respectively, $\left\langle u, \bar{\partial}_{\widetilde{\omega}}^{*} v\right\rangle_{X_{d}, \widetilde{\omega}}$ ) for every $d$.

It remains to show that the second term of the right-hand side in equality (2.1) converges to $\left(\left(u,(\bar{\partial} \Phi)^{*} v\right)\right)_{\partial X_{d}, \widetilde{\omega}}$ for almost all $d$. By Cauchy-Schwarz's inequality and Lemma 2.2, the integrand of the second term can be estimated as follows:

$$
\left|\left\langle\theta_{k} u,(\bar{\partial} \Phi)^{*} v\right\rangle_{\widetilde{\omega}}\right| \leqslant\left|\theta_{k} u\right|_{\widetilde{\omega}}\left|(\bar{\partial} \Phi)^{*} v\right|_{\widetilde{\omega}} \leqslant C \sup _{\partial X_{d}}\left(|\bar{\partial} \Phi|_{\widetilde{\omega}}\right)|u|_{\widetilde{\omega}}|v|_{\widetilde{\omega}} .
$$

We may assume $\widetilde{\omega} \geqslant \bar{\omega}$ for some positive $(1,1)$-form $\bar{\omega}$ on $X$ since $\widetilde{\omega}$ is complete on $Y$. Then, the inequality $|\bar{\partial} \Phi|_{\widetilde{\omega}} \leqslant|\bar{\partial} \Phi|_{\bar{\omega}}$ holds by Lemma 2.2. In particular, the sup-norm $\sup _{\partial X_{d}}|\bar{\partial} \Phi|_{\widetilde{\omega}}$ is finite since the function $\Phi$ is smooth on $X$ (not $Y$ ). If the integral of $|u|_{\widetilde{\omega}}|v|_{\tilde{\omega}}$ on $\partial X_{d}$ is finite, the integral $\left(\left(\theta_{k} u,(\bar{\partial} \Phi)^{*} v\right)\right)_{\partial X_{d}, \widetilde{\omega}}$ converges to $\left.\left(u,(\bar{\partial} \Phi)^{*} v\right)\right)_{\partial X_{d}, \widetilde{\omega}}$ by the bounded Lebesgue convergence theorem. To prove that the integral of $|u|_{\widetilde{\omega}}|v|_{\widetilde{\omega}}$ on $\partial X_{d}$ is finite for almost all $d$, we apply Fubini's theorem and Hölder's inequality to obtain

$$
\begin{aligned}
\int_{d \in(c-a, c+a)}\left(\int_{\partial X_{d}}|u|_{\widetilde{\omega}}|v|_{\widetilde{\omega}} d S_{\widetilde{\omega}}\right) d \Phi & =\int_{\{c-a<\Phi<c+a\}}|u|_{\widetilde{\omega}}|v|_{\widetilde{\omega}} d V_{\widetilde{\omega}} \\
& \leqslant\left(\int_{\{c-a<\Phi<c+a\}}|u|_{\widetilde{\omega}}^{2} d V_{\widetilde{\omega}}\right)^{1 / 2}\left(\int_{\{c-a<\Phi<c+a\}}|v|_{\widetilde{\omega}}^{2} d V_{\widetilde{\omega}}\right)^{1 / 2} .
\end{aligned}
$$

Here we used the equality $d \Phi \wedge d S_{\widetilde{\omega}}=d V_{\widetilde{\omega}}$. Since the right-hand side is finite from $\|u\|_{\widetilde{\omega}}<\infty$ and $\|v\|_{\widetilde{\omega}}<\infty$, the integral $\int_{\partial X_{d}}|u|_{\widetilde{\omega}}|v|_{\widetilde{\omega}} d S_{\widetilde{\omega}}$ should be finite for almost all $d$ in $(c-a, c+a)$. This completes the proof.

\subsection{Singular Hermitian metrics and multiplier ideal sheaves}

We recall the definitions of singular Hermitian metrics and curvature. Fix a smooth (Hermitian) metric $g$ on $F$. 


\section{INJECTIVITY THEOREMS FOR HIGHER DIRECT IMAGES}

Definition 2.7 (Singular Hermitian metrics and curvature). (1) For an $L_{\mathrm{loc}}^{1}$-function $\varphi$ on a complex manifold $X$, the Hermitian metric $h$ defined by

$$
h:=g e^{-2 \varphi}
$$

is called a singular Hermitian metric on $F$. Furthermore, the function $\varphi$ is called the weight of $F$ with respect to the fixed smooth metric $g$.

(2) The curvature $\sqrt{-1} \Theta_{h}(F)$ associated with $h$ is defined by

$$
\sqrt{-1} \Theta_{h}(F):=\sqrt{-1} \Theta_{g}(F)+2 \sqrt{-1} \partial \bar{\partial} \varphi,
$$

where $\sqrt{-1} \Theta_{g}(F)$ is the Chern curvature of $g$.

For simplicity, we refer to the singular Hermitian metric as the singular metric. In this paper, we consider only a singular metric $h$ such that $\sqrt{-1} \Theta_{h}(F) \geqslant \gamma$ holds for some smooth $(1,1)$ form $\gamma$ on $X$. Then, the weight function $\varphi$ is actually a quasi-plurisubharmonic (quasi-psh for short) function. In particular, the function $\varphi$ is upper semi-continuous. Moreover, the multiplier ideal sheaf defined below is coherent by a theorem of Nadel.

Definition 2.8 (Multiplier ideal sheaves). Let $h$ be a singular metric on $F$ such that $\sqrt{-1} \Theta_{h}(F)$ $\geqslant \gamma$ for some smooth $(1,1)$-form $\gamma$ on $X$. The ideal sheaf $\mathcal{I}(h)$, defined to be

$$
\mathcal{I}(h)(B):=\mathcal{I}(\varphi)(B):=\left\{f \in \mathcal{O}_{X}(B)|| f \mid e^{-\varphi} \in L_{\mathrm{loc}}^{2}(B)\right\}
$$

for every open set $B \subset X$, is called the multiplier ideal sheaf associated with $h$.

In Step 1, we approximate a given singular metric by singular metrics that are smooth on a Zariski open set. The following theorem is a reformulation of the equisingular approximation, which is proved by a slight variation on the proof of [DPS01, Theorem 2.3].

TheOREm 2.9 ([DPS01, Theorem 2.3]). Let $\omega$ be a positive $(1,1)$-form on a complex manifold $X$ and $(F, h)$ be a singular Hermitian line bundle on $X$. Assume that $\sqrt{-1} \Theta_{h}(F) \geqslant \gamma$ holds for a smooth (1,1)-form $\gamma$ on $X$. Then, for a relatively compact set $K \Subset X$, there exist singular metrics $\left\{h_{\varepsilon}\right\}_{1 \gg \varepsilon>0}$ on $\left.F\right|_{K}$ with the following properties:

(a) The metric $h_{\varepsilon}$ is smooth on $K \backslash Z_{\varepsilon}$ for some proper subvariety $Z_{\varepsilon}$ in $K$.

(b) The inequalities $h_{\varepsilon^{\prime \prime}} \leqslant h_{\varepsilon^{\prime}} \leqslant h$ hold on $K$ for any $0<\varepsilon^{\prime}<\varepsilon^{\prime \prime}$.

(c) We have $\mathcal{I}(h)=\mathcal{I}\left(h_{\varepsilon}\right)$ on $K$.

(d) We have $\sqrt{-1} \Theta_{h_{\varepsilon}}(F) \geqslant \gamma-\varepsilon \omega$ on $K$.

Remark 2.10. For a complete positive $(1,1)$-form $\omega_{K}$ on $K$, we may assume that property (d) holds for $\omega_{K}$; that is, $\sqrt{-1} \Theta_{h_{\varepsilon}}(F) \geqslant \gamma-\varepsilon \omega_{K}$ holds on $K$. This fact easily follows since $\omega_{K} \geqslant a \omega$ holds on $K$ for some $a>0$.

Proof of Theorem 2.9. In the case where $K=X$ and $X$ is compact, the theorem has been proved in [DPS01]. In [DPS01], we essentially use the assumption of $X$ being compact only when we take a finite cover of $X$ with inequality [DPS01, (2.1)]. Since $K$ is a relatively compact in $X$, for a given $\varepsilon>0$, we can take a finite open cover of $K$ by open balls $B_{i}:=\left\{\left|z^{(i)}\right|<r_{i}\right\}$ with a local coordinate $z^{(i)}$ satisfying inequality [DPS01, (2.1)]. Then, the same argument works by suitably replacing $X$ in [DPS01] with $K$. Indeed, uniform estimates [DPS01, (2.4)-(2.6)] can be checked in the same way since it is sufficient to consider only $B_{i}$ in this step. Furthermore, the function $\psi_{\varepsilon, \nu}$ can be defined on a neighborhood of $K$; see [DPS01, right before (2.7)]. Therefore, we can repeat the same argument. 


\section{S. Matsumura}

\subsection{Fréchet spaces}

In this subsection, we summarize fundamental facts on Hilbert spaces and Fréchet spaces. We give proofs for the reader's convenience.

Lemma 2.11. Let $L$ be a closed subspace in a Hilbert space $\mathcal{H}$. Then, the space $L$ is closed with respect to the weak topology of $\mathcal{H}$; that is, if a sequence $\left\{w_{k}\right\}_{k=1}^{\infty}$ in $L$ weakly converges to $w$, then the weak limit $w$ belongs to $L$.

Proof. By the orthogonal decomposition, there exists a closed subspace $M$ satisfying $L=M^{\perp}$. Then, we obtain that $0=\left\langle\left\langle w_{k}, v\right\rangle_{\mathcal{H}} \rightarrow\left\langle\langle w, v\rangle_{\mathcal{H}}\right.\right.$ as $k \rightarrow \infty$ for every $v \in M$. Therefore, we have $w \in M^{\perp}=L$.

Lemma 2.12. Let $\varphi: \mathcal{H}_{1} \rightarrow \mathcal{H}_{2}$ be a bounded operator (continuous linear map) between Hilbert spaces $\mathcal{H}_{1}$ and $\mathcal{H}_{2}$. If $\left\{w_{k}\right\}_{k=1}^{\infty}$ weakly converges to $w$ in $\mathcal{H}_{1}$, then $\left\{\varphi\left(w_{k}\right)\right\}_{k=1}^{\infty}$ weakly converges to $\varphi(w)$ in $\mathcal{H}_{2}$.

Proof. By taking the adjoint operator $\varphi^{*}$, we obtain

$$
\left\langle\varphi\left(w_{k}\right), v\right\rangle_{\mathcal{H}_{2}}=\left\langle\left\langle w_{k}, \varphi^{*}(v)\right\rangle_{\mathcal{H}_{1}} \rightarrow\left\langle w, \varphi^{*}(v)\right\rangle_{\mathcal{H}_{1}}=\langle\varphi(w), v\rangle_{\mathcal{H}_{2}}\right.
$$

for every $v \in \mathcal{H}_{2}$. This completes the proof.

Lemma 2.13. Let $\varphi: \mathcal{H} \rightarrow \mathcal{F}$ be a compact operator from a Hilbert space $\mathcal{H}$ to a Fréchet space $\mathcal{F}$. Assume that $\mathcal{F}$ is the inverse limit of Hilbert spaces. If $\left\{w_{k}\right\}_{k=1}^{\infty}$ weakly converges to $w$ in $\mathcal{H}$, then $\left\{\varphi\left(w_{k}\right)\right\}_{k=1}^{\infty}$ converges to $\varphi(w)$ in $\mathcal{F}$.

Proof. By assumption, there exist Hilbert spaces $\mathcal{F}_{m}$ such that $\mathcal{F}=\lim \mathcal{F}_{m}$. The induced operator $\varphi_{m}: \mathcal{H} \rightarrow \mathcal{F} \rightarrow \mathcal{F}_{m}$ is a compact operator; hence the vector $\varphi_{m}\left(\overleftarrow{w_{k}}\right)$ converges to $\varphi_{m}(w)$ in the Hilbert space $\mathcal{F}_{m}$. This means that $\varphi\left(w_{k}\right)$ converges to $\varphi(w)$ in $\mathcal{F}$.

\subsection{De Rham-Weil isomorphisms}

In this subsection, we observe the de Rham-Weil isomorphism from the $\bar{\partial}$-cohomology to the Čech cohomology, and we give a refinement of [Mat18a, Section 5] for our purpose. The contents of this subsection (including [Mat18a, Section 5]) may be known to specialists, but we will explain the details for the reader's convenience.

Throughout this subsection, let $\omega$ be a Kähler form on a complex manifold $X$ and $(F, h)$ be a singular Hermitian line bundle on $X$ such that $\sqrt{-1} \Theta_{h}(F) \geqslant-\omega$. Fix a locally finite open cover $\mathcal{U}:=\left\{B_{i}\right\}_{i \in I}$ of $X$ by Stein open sets $B_{i} \Subset X$. We consider the set of $q$-cochains $C^{q}\left(\mathcal{U}, K_{X} \otimes F \otimes \mathcal{I}(h)\right)$ with coefficients in $K_{X} \otimes F \otimes \mathcal{I}(h)$ calculated by $\mathcal{U}$ and the coboundary operator $\mu$ defined to be

$$
\mu\left\{\alpha_{i_{0} \ldots i_{q}}\right\}_{i_{0} \ldots i_{q}}:=\left\{\left.\sum_{\ell=0}^{q+1}(-1)^{\ell} \alpha_{i_{0} \ldots \hat{i}_{\ell} \ldots i_{q+1}}\right|_{B_{i_{0} \ldots i_{q+1}}}\right\}_{i_{0} \ldots i_{q+1}}
$$

for every $q$-cochain $\left\{\alpha_{i_{0} \ldots i_{q}}\right\}_{i_{0} \ldots i_{q}}$, where $B_{i_{0} \ldots i_{q+1}}:=B_{i_{0}} \cap B_{i_{1}} \cap \cdots \cap B_{i_{q+1}}$. In this paper, we will omit the notation of the restriction, the subscript $i_{0} \ldots i_{q}$, and so on. The semi-norm $p_{K_{i_{0} \ldots i_{q}}}(\bullet)$ is defined to be

$$
p_{K_{i_{0} \ldots i_{q}}}\left(\left\{\alpha_{i_{0} \ldots i_{q}}\right\}\right)^{2}:=\int_{K_{i_{0} \ldots i_{q}}}\left|\alpha_{i_{0} \ldots i_{q}}\right|_{h, \omega}^{2} d V_{\omega}
$$

for a $q$-cochain $\left\{\alpha_{i_{0} \ldots i_{q}}\right\}$ and a relatively compact set $K_{i_{0} \ldots i_{q}} \Subset B_{i_{0} \ldots i_{q}}$. Note that the semi-norm $p_{K_{i_{0} \ldots i_{q}}}(\bullet)$ does not depend on the choice of $\omega$ (see Lemma 2.2). The set of $q$-cochains can be 


\section{INJECTIVITY THEOREMS FOR HIGHER DIRECT IMAGES}

regarded as a topological vector space by the family of the semi-norms $\left\{p_{K_{i_{0} \ldots i_{q}}}(\bullet)\right\}_{K_{i_{0} \ldots i_{q}} \Subset B_{i_{0} \ldots i_{q}}}$. We have the following lemma.

Lemma 2.14. The set of $q$-cochains $C^{q}\left(\mathcal{U}, K_{X} \otimes F \otimes \mathcal{I}(h)\right)$ and the set of $q$-cocycles $Z^{q}\left(\mathcal{U}, K_{X} \otimes\right.$ $F \otimes \mathcal{I}(h)):=$ Ker $\mu$ are Fréchet spaces with respect to the semi-norms $\left\{p_{K_{i_{0} \ldots i_{q}}}(\bullet)\right\}_{K_{i_{0} \ldots i_{q}} \Subset B_{i_{0} \ldots i_{q}}}$. Moreover, if $X$ is holomorphically convex, then the set of $q$-coboundaries $B^{q}\left(\mathcal{U}, K_{X} \otimes F \otimes \mathcal{I}(h)\right):=$ $\operatorname{Im} \mu$ is also a Fréchet space.

Proof. Note that the topology of coherent ideal sheaves induced by the local sup-norms $\sup _{K_{i_{0} \ldots i_{q}}}(\bullet)$ is equivalent to the topology induced by the local $L^{2}$-norms $p_{K_{i_{0} \ldots i_{q}}}(\bullet)$ (see, for example, [GR65, Theorem 2, Section D, Chapter II] or [Mat18a, Lemma 5.2, Theorem 5.3, Lemma 5.7]). We can easily see that the metric induced by $p_{K_{i_{0} \ldots i_{q}}}(\bullet)$ is complete, and thus $C^{q}\left(\mathcal{U}, K_{X} \otimes F \otimes \mathcal{I}(h)\right)$ is a Fréchet space. It follows that $Z^{q}\left(\mathcal{U}, K_{X} \otimes F \otimes \mathcal{I}(h)\right)=\operatorname{Ker} \mu$ is a closed subspace (in particular, a Fréchet space) since the coboundary operator $\mu$ is continuous. For the latter conclusion, we consider the Cech cohomology group of $C^{q}\left(\mathcal{U}, K_{X} \otimes F \otimes \mathcal{I}(h)\right)$,

$$
\check{H}^{q}\left(\mathcal{U}, K_{X} \otimes F \otimes \mathcal{I}(h)\right):=\frac{\operatorname{Ker} \mu}{\operatorname{Im} \mu} .
$$

When $X$ is holomorphically convex, there exists a proper holomorphic map $\pi: X \rightarrow \Delta$ to a Stein space $\Delta$. For a coherent sheaf $\mathcal{F}$ on $X$, the natural morphism

$$
\pi_{*}: H^{q}(X, \mathcal{F}) \rightarrow H^{0}\left(\Delta, R^{q} \pi_{*} \mathcal{F}\right)
$$

is an isomorphism of topological vector spaces (see, for example, [Pri71, Lemma II.1]). In particular, the Čech cohomology group $\check{H}^{q}\left(\mathcal{U}, K_{X} \otimes F \otimes \mathcal{I}(h)\right)$ is a separated topological vector space when $X$ is holomorphically convex; hence $B^{q}\left(\mathcal{U}, K_{X} \otimes F \otimes \mathcal{I}(h)\right)=\operatorname{Im} \mu$ is closed.

We observe the $\bar{\partial}$-cohomology group of $L^{2}$-spaces defined on Zariski open sets equipped with suitable Kähler forms. Let $Z$ be a proper subvariety on $X$ and $\widetilde{\omega}$ be a Kähler form on the Zariski open set $Y:=X \backslash Z$ with the following properties:

(B) We have $\widetilde{\omega} \geqslant \omega$ on $Y=X \backslash Z$.

(C) For every point $p \in X$, there exists a bounded function $\Psi$ on an open neighborhood of $p$ such that $\widetilde{\omega}=\sqrt{-1} \partial \bar{\partial} \Psi$.

The important point here is that $\widetilde{\omega}$ locally admits a bounded potential function on $X$ (not $Y$ ). We define the local $L^{2}$-space of $F$-valued $(p, q)$-forms as follows:

$$
L_{(2, \mathrm{loc})}^{p, q}(F)_{h, \widetilde{\omega}}:=\left\{u \mid u \text { is an } F \text {-valued }(p, q) \text {-form with }\|u\|_{K, h, \widetilde{\omega}}<\infty \text { for every } K \Subset X\right\},
$$

where $\|u\|_{K, h, \omega}$ is the $L^{2}$-norm on a relatively compact set $K \Subset X$, that is,

$$
\|u\|_{K, h, \widetilde{\omega}}^{2}:=\int_{K \backslash Z}|u|_{h, \widetilde{\omega}}^{2} d V_{\widetilde{\omega}} .
$$

For the proof of Proposition 2.19, we concretely construct the de Rham-Weil isomorphism from the $\bar{\partial}$-cohomology to the Cech cohomology in Proposition 2.16. This construction plays an important role in the proof of the main results. To construct the de Rham-Weil isomorphism, we locally solve the $\bar{\partial}$-equation by Lemma 2.15, which is obtained from the standard technique of the theory of Kodaira-Andreotti-Vesentini-Hörmander [AV61, AV65, Hör65, Kod53]. The readers can check the proof of Lemma 2.15 in [Mat18a, Lemma 5.4] with the same notation. We need to generalize Lemma 2.15 to Lemma 3.8 for the proof of the main results. 


\section{S. Matsumura}

LEMMA 2.15. In the same situation as above, we assume that $B$ is a sufficiently small Stein open set in $X$. Then, for an arbitrary $U \in \operatorname{Ker} \bar{\partial} \subset L_{(2)}^{n, q}(B \backslash Z, F)_{h, \widetilde{\omega}}$, there exist $V \in L_{(2)}^{n, q-1}(B \backslash Z, F)_{h, \widetilde{\omega}}$ and a positive constant $C$ (depending only on $\Psi$ and $q$ ) such that

$$
\bar{\partial} V=U \quad \text { and } \quad\|V\|_{h, \widetilde{\omega}} \leqslant C\|U\|_{h, \widetilde{\omega}} .
$$

Proposition 2.16. We consider the same situation as above. That is, we consider a singular Hermitian line bundle $(F, h)$ on a Kähler manifold $(X, \omega)$ such that $\sqrt{-1} \Theta_{h}(F) \geqslant-\omega$ and a Kähler form $\widetilde{\omega}$ on a Zariski open set $Y$ satisfying properties $(B)$ and $(C)$. Then, there exist continuous maps

$$
\begin{aligned}
& f: \operatorname{Ker} \bar{\partial} \text { in } L_{(2, \text { loc })}^{n, q}(F)_{h, \widetilde{\omega}} \rightarrow \operatorname{Ker} \mu \quad \text { in } C^{q}\left(\mathcal{U}, K_{X} \otimes F \otimes \mathcal{I}(h)\right), \\
& g: \operatorname{Ker} \mu \text { in } C^{q}\left(\mathcal{U}, K_{X} \otimes F \otimes \mathcal{I}(h)\right) \rightarrow \operatorname{Ker} \bar{\partial} \quad \text { in } L_{(2, \text { loc })}^{n, q}(F)_{h, \widetilde{\omega}}
\end{aligned}
$$

satisfying the following properties:

- The map $f$ induces the isomorphism

$$
\bar{f}: \frac{\operatorname{Ker} \bar{\partial}}{\operatorname{Im} \bar{\partial}} \text { of } L_{(2, \text { loc })}^{n, q}(F)_{h, \widetilde{\omega}} \cong \frac{\operatorname{Ker} \mu}{\operatorname{Im} \mu} \text { of } C^{q}\left(\mathcal{U}, K_{X} \otimes F \otimes \mathcal{I}(h)\right) .
$$

- The map $g$ induces the isomorphism

$$
\bar{g}: \frac{\operatorname{Ker} \mu}{\operatorname{Im} \mu} \text { of } C^{q}\left(\mathcal{U}, K_{X} \otimes F \otimes \mathcal{I}(h)\right) \cong \frac{\operatorname{Ker} \bar{\partial}}{\operatorname{Im} \bar{\partial}} \text { of } L_{(2, \text { loc })}^{n, q}(F)_{h, \widetilde{\omega}} .
$$

- The map $\bar{f}$ is the inverse of $\bar{g}$.

Proof. The construction is essentially the same as the standard de Rham-Weil isomorphism. We briefly review only the construction of $f$ and $g$. See [Mat18a, Proposition 5.5] and [Fuj13, Lemma 3.20] for more details.

We first define $f(U)$ for $U \in \operatorname{Ker} \bar{\partial} \subset L_{(2, \text { loc })}^{n, q}(F)_{h, \widetilde{\omega}}$ by using the local solution of the $\bar{\partial}$ equation with minimum $L^{2}$-norm. We consider the $\bar{\partial}$-equation $\bar{\partial} \beta_{i_{0}}=\left.U\right|_{B_{i_{0}} \backslash Z}$ on $B_{i_{0}} \backslash Z$. Then, we can take the solution $\beta_{i_{0}}$ whose $L^{2}$-norm is minimum among all solutions. Further, we can see from Lemma 2.15 that $\left\|\beta_{i_{0}}\right\|_{h, \widetilde{\omega}} \leqslant C\|U\|_{B_{i_{0}}, h, \widetilde{\omega}}$ for some constant $C$ (independent of $U$ ). Next, we take the solution of the $\bar{\partial}$-equation $\bar{\partial} \beta_{i_{0} i_{1}}=\beta_{i_{1}}-\beta_{i_{0}}$ on $B_{i_{0} i_{1}} \backslash Z$ with minimum $L^{2}$-norm. By Lemma 2.15, we have

$$
\begin{aligned}
\left\|\beta_{i_{0} i_{1}}\right\|_{h, \widetilde{\omega}} & \leqslant D\left\|\beta_{i_{1}}-\beta_{i_{0}}\right\|_{B_{i_{0} i_{1}}, h, \widetilde{\omega}} \\
& \leqslant D\left(\left\|\beta_{i_{1}}\right\|_{h, \widetilde{\omega}}+\left\|\beta_{i_{0}}\right\|_{h, \widetilde{\omega}}\right) \\
& \leqslant C D\left(\|U\|_{B_{i_{0}}, h, \widetilde{\omega}}+\|U\|_{B_{i_{1}}, h, \widetilde{\omega}}\right)
\end{aligned}
$$

for some constant $D$. By repeating this process, we can obtain the $F$-valued $(n, q-k-1)$-forms $\beta_{i_{0} \ldots i_{k}}$ on $B_{i_{0} \ldots i_{k}} \backslash Z$ satisfying

$$
\left\{\begin{array}{l}
\bar{\partial}\left\{\beta_{i_{0}}\right\}=\left\{\left.U\right|_{B_{i_{0}} \backslash Z}\right\} \\
\bar{\partial}\left\{\beta_{i_{0} i_{1}}\right\}=\mu\left\{\beta_{i_{0}}\right\} \\
\bar{\partial}\left\{\beta_{i_{0} i_{1} i_{2}}\right\}=\mu\left\{\beta_{i_{0} i_{1}}\right\}, \\
\quad \vdots \\
\bar{\partial}\left\{\beta_{i_{0} \ldots i_{q-1}}\right\}=\mu\left\{\beta_{i_{0} \ldots i_{q-2}}\right\} .
\end{array}\right.
$$




\section{INJECTIVITY THEOREMS FOR HIGHER DIRECT IMAGES}

Then $\mu\left\{\beta_{i_{0} \ldots i_{q-1}}\right\}=:\left\{\beta_{i_{0} \ldots i_{q}}\right\}$ is a $q$-cocycle of $\bar{\partial}$-closed $F$-valued $(n, 0)$-forms on $B_{i_{0} \ldots i_{q}} \backslash Z$ such that $\left\|\beta_{i_{0} \ldots i_{q}}\right\|_{h, \widetilde{\omega}}<\infty$. Note that $\left\|\beta_{i_{0} \ldots i_{q}}\right\|_{h, \widetilde{\omega}}=\left\|\beta_{i_{0} \ldots i_{q}}\right\|_{h, \omega}$ by Lemma 2.2. The $F$-valued $(n, 0)$-form $\beta_{i_{0} \ldots i_{q}}$ can be locally regarded as a holomorphic function and can be extended from $B_{i_{0} \ldots i_{q}} \backslash Z$ to $B_{i_{0} \ldots i_{q}}$ by the Riemann extension theorem. This implies that $\mu\left\{\beta_{i_{0} \ldots i_{q-1}}\right\}=\left\{\beta_{i_{0} \ldots i_{q}}\right\}$ determines a $q$-cocycle in $C^{q}\left(\mathcal{U}, K_{X} \otimes F \otimes \mathcal{I}(h)\right)$. We define $f$ by $f(U):=\mu\left\{\beta_{i_{0} \ldots i_{q-1}}\right\}$.

Remark 2.17. By construction, we can obtain the $L^{2}$-estimate

$$
\left\|\beta_{i_{0} \ldots i_{k}}\right\|_{h, \widetilde{\omega}} \leqslant C \sum_{m=i_{0}, i_{1}, \ldots, i_{k}}\|U\|_{B_{m}, h, \widetilde{\omega}}
$$

for some constant $C$. Here $C$ depends only on a constant appearing when we solve the $\bar{\partial}$-equation by the $L^{2}$-method (see Lemma 2.15).

Proof of Proposition 2.16, continued. To define $g$, we fix a partition of unity $\left\{\rho_{i}\right\}_{i \in I}$ of $\mathcal{U}=$ $\left\{B_{i}\right\}_{i \in I}$. For a $q$-cocycle $\alpha=\left\{\alpha_{i_{0} \ldots i_{q}}\right\} \in C^{q}\left(\mathcal{U}, K_{X} \otimes F \otimes \mathcal{I}(h)\right)$, we define $g(\alpha)$ by

$$
\begin{aligned}
g(\alpha) & :=\bar{\partial}\left(\sum_{k_{q} \in I} \rho_{k_{q}} \bar{\partial}\left(\sum_{k_{q-1} \in I} \rho_{k_{q-1}} \cdots \bar{\partial}\left(\sum_{k_{3} \in I} \rho_{k_{3}} \bar{\partial}\left(\sum_{k_{2} \in I} \rho_{k_{2}} \bar{\partial}\left(\sum_{k_{1} \in I} \rho_{k_{1}} \alpha_{k_{1} \ldots k_{q} i_{0}}\right)\right)\right)\right)\right) \\
& =\sum_{k_{q} \in I} \bar{\partial} \rho_{k_{q}} \wedge \sum_{k_{q-1} \in I} \bar{\partial} \rho_{k_{q-1}} \wedge \cdots \wedge \sum_{k_{3} \in I} \bar{\partial} \rho_{k_{3}} \wedge \sum_{k_{2} \in I} \bar{\partial} \rho_{k_{2}} \wedge \bar{\partial}\left(\sum_{k_{1} \in I} \rho_{k_{1}} \alpha_{k_{1} \ldots k_{q} i_{0}}\right) .
\end{aligned}
$$

This determines the $\bar{\partial}$-closed $F$-valued $(n, q)$-form with locally bounded $L^{2}$-norm.

The following proposition can be deduced from Proposition 2.16.

Proposition 2.18. In the same situation as in Proposition 2.16, we assume that $X$ is holomorphically convex. Then Im $\bar{\partial}$ is a closed subspace in $L_{(2, \text { loc })}^{n, q}(F)_{h, \widetilde{\omega}}$. In particular, the $\bar{\partial}$-cohomology group $\operatorname{Ker} \bar{\partial} / \operatorname{Im} \bar{\partial}$ of $L_{(2, \text { loc })}^{n, q}(F)_{h, \widetilde{\omega}}$ is a Fréchet space.

Proof. The Čech cohomology group is a separated topological vector space (see Lemma 2.14). Thus, it follows from Proposition 2.16 that $\operatorname{Im} \bar{\partial}$ is a closed subspace.

We close this subsection with the following proposition.

Proposition 2.19. In the same situation as in Proposition 2.16, we assume that $X$ is holomorphically convex. Then, the following composite map is a compact operator:

$$
\operatorname{Ker} \bar{\partial} \text { in } L_{(2, \text { loc })}^{n, q}(F)_{h, \widetilde{\omega}} \longrightarrow \frac{\operatorname{Ker} \bar{\partial}}{\operatorname{Im} \bar{\partial}} \text { in } L_{(2, \text { loc })}^{n, q}(F)_{h, \widetilde{\omega}}
$$

In the proof of Proposition 2.19, we use the construction of $f$ and $g$ from Proposition 2.16. For the reader's convenience, we first give a proof for the case $q=1$.

Proof of Proposition 2.19 for the case $q=1$. We take a bounded sequence $\left\{U_{\ell}\right\}_{\ell=1}^{\infty}$ in Ker $\bar{\partial} \subset$ $L_{(2, \text { loc })}^{n, 1}(Y, F)_{h, \widetilde{\omega}}$; that is, for any $K \Subset X$, we have $\left\|U_{\ell}\right\|_{h, \widetilde{\omega}} \leqslant C_{K}$ for some constant $C_{K}$ (independent of $\ell$ ). For the restriction $U_{\ell, i}:=\left.U_{\ell}\right|_{B_{i} \backslash Z}$, by solving the $\bar{\partial}$-equation $\bar{\partial} \beta_{\ell, i}=U_{\ell, i}$ on $B_{i} \backslash Z$, we obtain $\beta_{\ell, i}$ and a constant $C$ independent of $\ell$ with the following properties:

$$
\bar{\partial} \beta_{\ell, i}=U_{\ell, i} \quad \text { on } B_{i} \backslash Z \quad \text { and } \quad\left\|\beta_{\ell, i}\right\|_{h, \widetilde{\omega}} \leqslant C\left\|U_{\ell, i}\right\|_{B_{i}, h, \widetilde{\omega}} .
$$

Here we used the assumption that $\widetilde{\omega}$ locally admits a bounded potential function $\Psi$ (see Lemma 2.15). In particular, the $F$-valued $(n, 0)$-form $\beta_{\ell, j}-\beta_{\ell, i}$ is $\bar{\partial}$-closed on $B_{i j} \backslash Z$, and it is 


\section{S. Matsumura}

$L^{2}$-bounded. Hence it can be extended to the $\bar{\partial}$-closed $F$-valued $(n, 0)$-form on $B_{i} \cap B_{j}$ by the Riemann extension theorem.

We now construct the $F$-valued $(n, 1)$-form $V_{\ell}$ such that $V_{\ell}$ determines the same cohomology class as $U_{\ell}$ and that $V_{\ell}$ converges to some $F$-valued $(n, 1)$-form, which completes the proof. By construction, the sup-norm $\sup _{K}\left|\beta_{\ell, j}-\beta_{\ell, i}\right|$ is uniformly bounded for every $K \Subset B_{i j}$. (Recall that the local sup-norm of holomorphic functions can be bounded by the $L^{2}$-norm.) Hence, by Montel's theorem, there exists a subsequence of $\left\{\beta_{\ell, j}-\beta_{\ell, i}\right\}_{\ell=1}^{\infty}$ that uniformly converges to some $\alpha_{i j}$ as $\ell \rightarrow \infty$ on every relatively compact set in $B_{i j}$. We use the same notation for this subsequence. This subsequence also converges to $\alpha_{i j}$ with respect to the local $L^{2}$-norms (see, for example, [Mat18a, Lemma 5.2]).

For a fixed partition of unity $\left\{\rho_{i}\right\}_{i \in I}$ of $\mathcal{U}$, we define the $F$-valued $(n, 1)$-form $V_{\ell, i}$ by

$$
V_{\ell, i}:=\bar{\partial}\left(\sum_{k \in I} \rho_{k}\left(\beta_{\ell, i}-\beta_{\ell, k}\right)\right)=\bar{\partial}\left(\beta_{\ell, i}-\sum_{k \in I} \rho_{k} \beta_{\ell, k}\right)=U_{\ell}-\bar{\partial}\left(\sum_{k \in I} \rho_{k} \beta_{\ell, k}\right) \quad \text { on } B_{i} \backslash Z \text {. }
$$

Since $\sum_{k \in I} \rho_{k} \beta_{\ell, k}$ is independent of $i$, the family $\left\{V_{\ell, i}\right\}_{i \in I}$ determines the $F$-valued $(n, 1)$-form $V_{\ell}$ globally defined on $Y$. Furthermore, the $F$-valued $(n, 1)$-form $V_{\ell}$ determines the same cohomology class as $U_{\ell}$. It is sufficient for the proof to show that $V_{\ell}$ converges to

$$
\sum_{k \in I} \bar{\partial}\left(\rho_{k} \alpha_{k i}\right)=\sum_{k \in I} \bar{\partial} \rho_{k} \wedge \alpha_{k i} \quad \text { in } L_{(2, \text { loc })}^{n, 1}(F)_{h, \tilde{\omega}} .
$$

For a given $K \Subset X$, the cardinality of $I_{K}$ defined by

$$
I_{K}:=\left\{i \in I \mid B_{i} \cap K \neq \emptyset\right\}
$$

is finite. Hence we obtain

$$
\begin{aligned}
\left\|V_{\ell}-\sum_{k \in I} \bar{\partial}\left(\rho_{k} \alpha_{k i}\right)\right\|_{K, h, \widetilde{\omega}} & \leqslant \sum_{i \in I_{K}}\left\|\sum_{k \in I} \bar{\partial} \rho_{k} \wedge\left(\beta_{\ell, i}-\beta_{\ell, k}-\alpha_{k i}\right)\right\|_{B_{i}, h, \widetilde{\omega}} \\
& \leqslant C_{2}\left\|\left(\beta_{\ell, i}-\beta_{\ell, k}-\alpha_{k i}\right)\right\|_{B_{i} \cap \operatorname{Supp} \rho_{k}, h, \widetilde{\omega}}
\end{aligned}
$$

for some constant $C_{2}>0$. Here we used $\left|\bar{\partial} \rho_{k}\right|_{\widetilde{\omega}} \leqslant\left|\bar{\partial} \rho_{k}\right|_{\omega}$ (see Lemma 2.2 and property (B)). By $K_{i} \cap \operatorname{Supp} \rho_{k} \Subset B_{i k}$, the right-hand side converges to zero as $\ell \rightarrow \infty$.

Proof of Proposition 2.19 for the general case. We take a bounded sequence $\left\{U_{\ell}\right\}_{\ell=1}^{\infty}$ in Ker $\bar{\partial} \subset$ $L_{(2, \mathrm{loc})}^{n, q}(Y, F)_{h, \widetilde{\omega}}$. Then, by the construction of $f$, we can obtain the $F$-valued $(n, q-k-1)$-forms $\beta_{\ell, i_{0} \ldots i_{k}}$ on $B_{i_{0} \ldots i_{k}} \backslash Z$ satisfying equality (2.4). Furthermore, we obtain

$$
f\left(U_{\ell}\right)=\mu\left\{\beta_{\ell, i_{0} \ldots i_{q-1}}\right\}=:\left\{\beta_{\ell, i_{0} \ldots i_{q}}\right\} \quad \text { and } \quad\left\|\beta_{\ell, i_{0} \ldots i_{q}}\right\|_{B_{i_{0} \ldots i_{q}}, h, \widetilde{\omega}} \leqslant C \sum_{m=i_{0}, i_{1}, \cdots, i_{q}}\left\|U_{\ell}\right\|_{B_{m}, h, \widetilde{\omega}} .
$$

Here $C$ is a positive constant independent of $\ell$ (see Remark 2.17). Then, in the same way as in the case $q=1$, we can take a subsequence of $\left\{f\left(U_{\ell}\right)\right\}_{\ell=1}^{\infty}$ converging to some $q$-cocycle $\alpha$ with respect to the local $L^{2}$-norms. We use the same notation $\left\{f\left(U_{\ell}\right)\right\}_{\ell=1}^{\infty}$ for this subsequence.

When we fix a partition of unity $\left\{\rho_{i}\right\}_{i \in I}$ of $\mathcal{U}$, we can define the map $g$ in Proposition 2.16. It follows that $g\left(f\left(U_{\ell}\right)\right)$ converges to $g(\alpha)$ in $L_{(2, \text { loc })}^{n, q}(F)_{h, \widetilde{\omega}}$ since the map $g$ is continuous. Furthermore, we can see from Proposition 2.16 that $g\left(f\left(U_{\ell}\right)\right)$ determines the same cohomology class as $U_{\ell}$. This completes the proof. 


\section{INJECTIVITY THEOREMS FOR HIGHER DIRECT IMAGES}

\section{Proof of the main results}

\subsection{Proof of Theorem 1.2}

This subsection is devoted to the proof of Theorem 1.2. The proof can be divided into five steps. Theorem 1.3 will be proved by slightly revising Step 5 (see Subsection 3.2). In the following proof, we often write (possibly different) positive constants as $C$ and use the same notation for suitably chosen subsequences.

Step 1 (Strategy of the proof). In this step, we fix the notation used in this subsection and reduce the proof to a local problem on $\Delta$. At the end of this step, the rough strategy of the proof will be explained, to help to understand the complicated and technical arguments.

The problem is local on $\Delta$, and thus we may assume that $\pi: X \rightarrow \Delta$ is a surjective proper holomorphic map from a Kähler manifold $X$ to a Stein subvariety $\Delta$ in $\mathbb{C}^{N}$. In particular, the manifold $X$ is holomorphically convex; hence, for a coherent sheaf $\mathcal{F}$ on $X$, the natural morphism

$$
\pi_{*}: H^{q}(X, \mathcal{F}) \rightarrow H^{0}\left(\Delta, R^{q} \pi_{*} \mathcal{F}\right)
$$

is an isomorphism of topological vector spaces (see, for example, [Pri71, Lemma II.1]). This indicates that it is sufficient to show that the multiplication map

$$
H^{q}\left(X, K_{X} \otimes F \otimes \mathcal{I}(h)\right) \stackrel{\otimes s}{\longrightarrow} H^{q}\left(X, K_{X} \otimes F^{m+1} \otimes \mathcal{I}\left(h^{m+1}\right)\right)
$$

is injective. The injectivity has already been proved when $X$ is compact [Mat18a]. The main difficulty arises from the fact of $X$ being non-compact.

By replacing $\Delta$ with a smaller disk, we may assume that $X$ is a relatively compact set in the initial ambient space. In particular, we may assume that the point-wise norm $|s|_{h^{m}}$ is bounded on $X$ by assumption and that $X$ admits a complete Kähler form since $X$ is a weakly pseudoconvex Kähler manifold. For a fixed complete Kähler form $\omega$ on $X$, by applying Theorem 2.9 and Remark 2.10 to $\gamma=0$, we can take a family of singular metrics $\left\{h_{\varepsilon}\right\}_{1 \gg \varepsilon>0}$ on $F$ with the following properties:

(a) The metric $h_{\varepsilon}$ is smooth on $X \backslash Z_{\varepsilon}$ for some proper subvariety $Z_{\varepsilon}$.

(b) The inequalities $h_{\varepsilon^{\prime \prime}} \leqslant h_{\varepsilon^{\prime}} \leqslant h$ hold on $X$ for any $0<\varepsilon^{\prime}<\varepsilon^{\prime \prime}$.

(c) We have $\mathcal{I}(h)=\mathcal{I}\left(h_{\varepsilon}\right)$ on $X$.

(d) We have $\sqrt{-1} \Theta_{h_{\varepsilon}}(F) \geqslant-\varepsilon \omega$ on $X$.

Remark 3.1. In the case $m>0$, it follows from $\sup |s|_{h^{m}}<\infty$ that the set $\{x \in X \mid \nu(h, x)>0\}$ is contained in the zero locus $s^{-1}(0)$, where $\nu(h, x)$ is the Lelong number of the weight of $h$ at $x$. Then, we can assume that $Z_{\varepsilon}$ is independent of $\varepsilon$. However, in the case $m=0$, the subvariety $Z_{\varepsilon}$ essentially depends on $\varepsilon$. This is different from [Mat18a] and one of the difficulties of the proof. To overcome this difficulty, we consider a complete Kähler form $\omega_{\varepsilon, \delta}$ on $Y_{\varepsilon}:=X \backslash Z_{\varepsilon}$ such that $\omega_{\varepsilon, \delta}$ converges to $\omega$ as $\delta \rightarrow 0$.

To apply the theory of harmonic integrals on the Zariski open set $Y_{\varepsilon}$, we first take a form $\omega_{\varepsilon}$ on $Y_{\varepsilon}$ with the following properties:

- The form $\omega_{\varepsilon}$ is complete Kähler on $Y_{\varepsilon}$.

- We have $\omega_{\varepsilon} \geqslant \omega$ on $Y_{\varepsilon}$.

- We have $\omega_{\varepsilon}=\sqrt{-1} \partial \bar{\partial} \Psi_{\varepsilon}$ for some bounded function $\Psi_{\varepsilon}$ on a neighborhood of every $p \in X$. 


\section{S. Matsumura}

See $\left[\right.$ Fuj13, 3.11] for the construction of $\omega_{\varepsilon}$. The key point here is the third property on the bounded potential function, which enables us to construct the de Rham-Weil isomorphism from the $\bar{\partial}$-cohomology group on $Y_{\varepsilon}$ to the Cech cohomology group on $X$ (see Proposition 2.16). We define the Kähler form $\omega_{\varepsilon, \delta}$ on $Y_{\varepsilon}$ by

$$
\omega_{\varepsilon, \delta}:=\omega+\delta \omega_{\varepsilon} \quad \text { for } \varepsilon \text { and } \delta \text { with } 0<\delta \ll \varepsilon .
$$

It is easy to see the following properties:

(A) The form $\omega_{\varepsilon, \delta}$ is complete Kähler on $Y_{\varepsilon}=X \backslash Z_{\varepsilon}$ for every $\delta>0$.

(B) We have $\omega_{\varepsilon, \delta} \geqslant \omega$ on $Y_{\varepsilon}$ for every $\delta>0$.

(C) For every point $p \in X$, there exists a bounded function $\Psi_{\varepsilon, \delta}=\Psi+\delta \Psi_{\varepsilon}$ on an open neighborhood of $p$ in $X$ such that $\sqrt{-1} \partial \bar{\partial} \Psi_{\varepsilon, \delta}=\omega_{\varepsilon, \delta}$ and $\lim _{\delta \rightarrow 0} \Psi_{\varepsilon, \delta}=\Psi$. Here $\Psi$ is a local potential function of $\omega$.

Remark 3.2. Strictly speaking, from Theorem 2.9 [DPS01, Theorem 2.3], we obtain a countable family $\left\{h_{\varepsilon_{k}}\right\}_{k=1}^{\infty}$ of singular metrics satisfying the above properties. In the proof, we actually consider only countable sequences $\left\{\varepsilon_{k}\right\}_{k=1}^{\infty}$ and $\left\{\delta_{\ell}\right\}_{\ell=1}^{\infty}$ conversing to zero, but we often use the notation $\varepsilon$ and $\delta$ for simplicity.

We define the function $\Phi$ on $X$ by

$$
\Phi:=\pi^{*} i^{*}\left(\left|z_{1}\right|^{2}+\left|z_{2}\right|^{2}+\cdots+\left|z_{N}\right|^{2}\right)
$$

where $i: \Delta \rightarrow \mathbb{C}^{N}$ is a local embedding of the Stein subvariety $\Delta$ and $\left(z_{1}, z_{2}, \ldots, z_{N}\right)$ is a coordinate system of $\mathbb{C}^{N}$. By construction, the function $\Phi$ is a psh function on $X$. Furthermore, since $\pi$ is a proper morphism, the function $\Phi$ is an exhaustive function on $X$ (that is, the level set $X_{c}:=\{x \in X \mid \Phi(x)<c\}$ is relatively compact in $X$ for every $c$ with $\left.c<\sup _{X} \Phi\right)$. By replacing $\Delta$ with a smaller disk, we may assume

$$
\sup _{X} \Phi<\infty \quad \text { and } \sup _{X}|d \Phi|_{\omega_{\varepsilon, \delta}}<C
$$

and also that $\Phi$ and $\omega_{\varepsilon, \delta}$ satisfy the assumptions in Proposition 2.3. Indeed, we can assume that $\Phi$ is defined on a neighborhood of $\partial X$ in the initial ambient space by taking a smaller $\Delta$. This indicates that $\sup _{X} \Phi<\infty$ and $\sup _{X}|d \Phi|_{\bar{\omega}}<\infty$ for some positive $(1,1)$-form $\bar{\omega}$ defined on the neighborhood of $\partial X$. We may assume $\omega_{\varepsilon, \delta} \geqslant \omega \geqslant \bar{\omega}$ since $\omega$ is a complete form on $X$. Then, from Lemma 2.2, we have $\sup _{X}|d \Phi|_{\omega_{\varepsilon, \delta}} \leqslant \sup _{X}|d \Phi|_{\bar{\omega}}<\infty$.

Let $A$ be a cohomology class in $H^{q}\left(X, K_{X} \otimes F \otimes \mathcal{I}(h)\right)$ such that

$$
s A=0 \in H^{q}\left(X, K_{X} \otimes F^{m+1} \otimes \mathcal{I}\left(h^{m+1}\right)\right) .
$$

Our goal is to prove that $A$ is actually the zero cohomology class.

We briefly explain the strategy of the proof with the above notation. In Step 2, by suitably choosing an increasing convex function $\chi: \mathbb{R} \rightarrow \mathbb{R}$, we represent $A$ by harmonic $L^{2}$-forms $u_{\varepsilon, \delta}$ with respect to $\omega_{\varepsilon, \delta}$ and the new metric $H_{\varepsilon}$ defined by $H_{\varepsilon}:=h_{\varepsilon} e^{-\chi \circ \Phi}$. In Step 3, we consider the level set $X_{c}:=\{x \in X \mid \Phi(x)<c\}$ and show that if the $L^{2}$-norm $\left\|s u_{\varepsilon, \delta}\right\|_{X_{c}, H_{\varepsilon} h_{\varepsilon}^{m}, \omega_{\varepsilon, \delta}}$ on $X_{c}$ converges to zero for almost all $c$, then $A$ is the zero cohomology class. To prove this convergence, in Step 4, we construct a solution $v_{\varepsilon, \delta}$ of the $\bar{\partial}$-equation $\bar{\partial} v_{\varepsilon, \delta}=s u_{\varepsilon, \delta}$ such that the $L^{2}$-norm $\left\|v_{\varepsilon, \delta}\right\|_{X_{c}, H_{\varepsilon}, \omega_{\varepsilon, \delta}}$ on $X_{c}$ is uniformly bounded. In Step 5 , we use the twisted Bochner-Kodaira-Nakano identity to 


\section{INJECTIVITY THEOREMS FOR HIGHER DIRECT IMAGES}

show that

$$
\begin{aligned}
\left\|s u_{\varepsilon, \delta}\right\|_{X_{c}, H_{\varepsilon} h_{\varepsilon}^{m}, \omega_{\varepsilon, \delta}}^{2} & =\left\langle s u_{\varepsilon, \delta}, \bar{\partial} v_{\varepsilon, \delta}\right\rangle_{X_{c}, H_{\varepsilon}} h_{\varepsilon}^{m}, \omega_{\varepsilon, \delta} \\
& =\left\langle\bar{\partial}^{*} s u_{\varepsilon, \delta}, v_{\varepsilon, \delta}\right\rangle_{X_{c}, H_{\varepsilon} h_{\varepsilon}^{m}, \omega_{\varepsilon, \delta}}+\left(\left((d \Phi)^{*} s u_{\varepsilon, \delta}, v_{\varepsilon, \delta}\right)\right)_{\partial X_{c}, H_{\varepsilon} h_{\varepsilon}^{m}, \omega_{\varepsilon, \delta} \rightarrow 0 .}
\end{aligned}
$$

Step 2 ( $L^{2}$-spaces and representations by harmonic forms). In this step, we construct harmonic $L^{2}$-forms $u_{\varepsilon, \delta}$ representing the cohomology class $A \in H^{q}\left(X, K_{X} \otimes F \otimes \mathcal{I}(h)\right)$ and prove Proposition 3.6, which states that if $u_{\varepsilon, \delta}$ converges to zero in a suitable sense, then $A$ is zero (that is, the proof is completed).

We first consider the standard de Rham-Weil isomorphism:

$$
H^{q}\left(X, K_{X} \otimes F \otimes \mathcal{I}(h)\right) \cong \frac{\operatorname{Ker} \bar{\partial}: L_{(2, \text { loc })}^{n, q}(F)_{h, \omega} \rightarrow L_{(2, \text { loc })}^{n, q+1}(F)_{h, \omega}}{\operatorname{Im} \bar{\partial}: L_{(2, \text { loc })}^{n, q-1}(F)_{h, \omega} \rightarrow L_{(2, \text { loc })}^{n, q}(F)_{h, \omega}},
$$

where $L_{(2, \text { loc })}^{n, \bullet}(F)_{h, \omega}$ is the set of $F$-valued $(n, \bullet)$-forms $f$ on $X$ with locally bounded $L^{2}$-norm (that is, the $L^{2}$-norm $\|f\|_{K, h, \omega}$ on $K$ with respect to $h$ and $\omega$ is finite for every relatively compact set $K \Subset X)$. By the above isomorphism, the cohomology class $A$ can be represented by a $\bar{\partial}$ closed $F$-valued $(n, q)$-form $u$ on $X$ with locally bounded $L^{2}$-norm. Our goal is to prove that $u \in \operatorname{Im} \bar{\partial} \subset L_{(2, \text { loc })}^{n, q}(F)_{h, \omega}$.

We want to represent the cohomology class $A$ by harmonic forms in $L^{2}$-spaces, but $u$ may not be globally $L^{2}$-integrable on $X$. For this reason, we construct a new metric on $F$ that makes $u$ globally $L^{2}$-integrable. Since $u$ is locally $L^{2}$-integrable and $\Phi$ is exhaustive, there exists an increasing convex function $\chi: \mathbb{R} \rightarrow \mathbb{R}$ such that the $L^{2}$-norm $\|u\|_{h e^{-\chi \circ \Phi, \omega}}$ on $X$ is finite. For simplicity, we set

$$
H:=h e^{-\chi \circ \Phi}, \quad H_{\varepsilon}:=h_{\varepsilon} e^{-\chi \circ \Phi}, \quad \text { and } \quad\|u\|_{\varepsilon, \delta}:=\|u\|_{H_{\varepsilon}, \omega_{\varepsilon, \delta}} .
$$

Then, we obtain the following inequality:

$$
\|u\|_{\varepsilon, \delta} \leqslant\|u\|_{H, \omega_{\varepsilon, \delta}} \leqslant\|u\|_{H, \omega}<\infty .
$$

Strictly speaking, the left-hand side should be $\left\|\left.u\right|_{Y_{\varepsilon}}\right\|_{\varepsilon, \delta}$, but we often omit the restriction symbol. The first inequality follows from property (b) of $h_{\varepsilon}$, and the second inequality follows from Lemma 2.2 and property (B) of $\omega_{\varepsilon, \delta}$. Here we used a special characteristic of the canonical bundle $K_{X}$ since the second inequality holds only for $(n, q)$-forms. The norm $\|u\|_{\varepsilon, \delta}$ is uniformly bounded since the right-hand side is independent of $\varepsilon$ and $\delta$.

We consider the $L^{2}$-space

$$
L_{(2)}^{n, q}(F)_{\varepsilon, \delta}:=L_{(2)}^{n, q}\left(Y_{\varepsilon}, F\right)_{H_{\varepsilon}, \omega_{\varepsilon, \delta}}
$$

on $Y_{\varepsilon}$ with respect to $H_{\varepsilon}$ and $\omega_{\varepsilon, \delta}$ (not $H$ and $\omega$ ). In general, we have the following orthogonal decomposition:

$$
L_{(2)}^{n, q}(F)_{\varepsilon, \delta}=\overline{\operatorname{Im} \bar{\partial}} \oplus \mathcal{H}_{\varepsilon, \delta}^{n, q}(F) \oplus \overline{\operatorname{Im} \bar{\partial}_{\varepsilon, \delta}^{*}},
$$

where - denotes the closure of $\bullet$ with respect to the $L^{2}$-topology and $\mathcal{H}_{\varepsilon, \delta}^{n, q}(F)$ denotes the set of harmonic $F$-valued $(n, q)$-forms on $Y_{\varepsilon}$, that is,

$$
\mathcal{H}_{\varepsilon, \delta}^{n, q}(F):=\left\{v \in L_{(2)}^{n, q}(F)_{\varepsilon, \delta} \mid \bar{\partial} v=\bar{\partial}_{\varepsilon, \delta}^{*} v=0\right\} .
$$

Note that (the maximal extension of) the formal adjoint $\bar{\partial}_{\varepsilon, \delta}^{*}$ agrees with the Hilbert space adjoint since $\omega_{\varepsilon, \delta}$ is complete for $\delta>0$ (see Lemma 2.4). The operator $\bar{\partial}$ also depends on $H_{\varepsilon}$ 


\section{S. Matsumura}

and $\omega_{\varepsilon, \delta}$ in the sense that the domain and range of the closed operator $\bar{\partial}$ depend on them, but we abbreviate $\bar{\partial}_{\varepsilon, \delta}$ to $\bar{\partial}$. The $F$-valued $(n, q)$-form $u$ belongs to $L_{(2)}^{n, q}(F)_{\varepsilon, \delta}$ by inequality (3.4); thus $u$ can be decomposed as follows:

$$
u=w_{\varepsilon, \delta}+u_{\varepsilon, \delta} \quad \text { for some } w_{\varepsilon, \delta} \in \overline{\operatorname{Im} \bar{\partial}} \text { and } u_{\varepsilon, \delta} \in \mathcal{H}_{\varepsilon, \delta}^{n, q}(F) \text { in } L_{(2)}^{n, q}(F)_{\varepsilon, \delta}
$$

from the above orthogonal decomposition. Note that the orthogonal projection of $u$ to $\overline{\operatorname{Im} \bar{\partial}_{\varepsilon, \delta}^{*}}$ is zero by the following lemma.

Lemma 3.3. If $u$ belongs to Ker $\bar{\partial}$, then the orthogonal projection of $u$ to $\overline{\operatorname{Im} \bar{\partial}_{\varepsilon, \delta}^{*}}$ is zero.

Proof. For an arbitrary element $\lim _{k \rightarrow \infty} \bar{\partial}_{\varepsilon, \delta}^{*} c_{k} \in \overline{\operatorname{Im} \bar{\partial}_{\varepsilon, \delta}^{*}}$, we have

$$
\left\langle u, \lim _{k \rightarrow \infty} \bar{\partial}_{\varepsilon, \delta}^{*} c_{k}\right\rangle_{\varepsilon, \delta}=\lim _{k \rightarrow \infty}\left\langle u, \bar{\partial}_{\varepsilon, \delta}^{*} c_{k}\right\rangle_{\varepsilon, \delta}=\lim _{k \rightarrow \infty}\left\langle\bar{\partial} u, c_{k}\right\rangle_{\varepsilon, \delta}=0 .
$$

This leads to the conclusion.

From now on, we consider a suitable limit of $u_{\varepsilon, \delta}$. We need to carefully choose the $L^{2}$-space since the $L^{2}$-space $L_{(2)}^{n, q}(F)_{\varepsilon, \delta}$ depends on $\varepsilon$ and $\delta$ although we have property (c). Note that $\{\varepsilon\}_{\varepsilon>0}$ and $\{\delta\}_{\delta>0}$ denote countable sequences converging to zero (see Remark 3.2). Let $\left\{\delta_{0}\right\}_{\delta_{0}>0}$ be another countable sequence converging to zero.

Proposition 3.4. There exist a subsequence $\left\{\delta_{\nu}\right\}_{\nu=1}^{\infty}$ of $\{\delta\}_{\delta>0}$ and $\alpha_{\varepsilon} \in L_{(2)}^{n, q}(F)_{H_{\varepsilon}, \omega}$ with the following properties:

- For any $\varepsilon, \delta_{0}>0$, as $\delta_{\nu} \rightarrow 0$,

$$
u_{\varepsilon, \delta_{\nu}} \text { converges to } \alpha_{\varepsilon} \text { with respect to the weak } L^{2} \text {-topology in } L_{(2)}^{n, q}(F)_{\varepsilon, \delta_{0}} \text {. }
$$

- For any $\varepsilon>0$, we have

$$
\left\|\alpha_{\varepsilon}\right\|_{H_{\varepsilon}, \omega} \leqslant \varliminf_{\delta_{0} \rightarrow 0}\left\|\alpha_{\varepsilon}\right\|_{\varepsilon, \delta_{0}} \leqslant \varliminf_{\delta_{\nu} \rightarrow 0}\left\|u_{\varepsilon, \delta_{\nu}}\right\|_{\varepsilon, \delta_{\nu}} \leqslant\|u\|_{H, \omega} .
$$

Remark 3.5. The subsequence $\left\{\delta_{\nu}\right\}_{\nu=1}^{\infty}$ does not depend on $\varepsilon$ and $\delta_{0}$. The $F$-valued form $\alpha_{\varepsilon}$ is independent of $\delta_{0}$ and $L^{2}$-integrable with respect to $H_{\varepsilon}$ and $\omega\left(\right.$ not $\left.\omega_{\varepsilon, \delta}\right)$.

Proof. For any $\varepsilon, \delta_{0}>0$, by taking $\delta$ with $\delta<\delta_{0}$, we have

$$
\left\|u_{\varepsilon, \delta}\right\|_{\varepsilon, \delta_{0}} \leqslant\left\|u_{\varepsilon, \delta}\right\|_{\varepsilon, \delta} \leqslant\|u\|_{\varepsilon, \delta} \leqslant\|u\|_{H, \omega}
$$

The first inequality follows from $\omega_{\varepsilon, \delta} \leqslant \omega_{\varepsilon, \delta_{0}}$ and Lemma 2.2. The second inequality follows since $u_{\varepsilon, \delta}$ is the orthogonal projection of $u$ with respect to $\varepsilon$ and $\delta$. The last inequality follows from inequality (3.4). These inequalities indicate that $\left\{u_{\varepsilon, \delta}\right\}_{\delta>0}$ is uniformly bounded in $L_{(2)}^{n, q}(F)_{\varepsilon, \delta_{0}}$. Hence there exist a subsequence $\left\{\delta_{\nu}\right\}_{\nu=1}^{\infty}$ of $\{\delta\}_{\delta>0}$ and $\alpha_{\varepsilon, \delta_{0}} \in L_{(2)}^{n, q}(F)_{\varepsilon, \delta_{0}}$ such that $u_{\varepsilon, \delta_{\nu}}$ converges to $\alpha_{\varepsilon, \delta_{0}}$ with respect to the weak $L^{2}$-topology in $L_{(2)}^{n, q}(F)_{\varepsilon, \delta_{0}}$. The choice of this subsequence $\left\{\delta_{\nu}\right\}_{\nu=1}^{\infty}$ depends on $\varepsilon$ and $\delta$, but by extracting a suitable subsequence, we can take a subsequence independent of $\varepsilon$ and $\delta_{0}$ by Cantor's diagonal argument.

We prove that $\alpha_{\varepsilon, \delta_{0}}$ does not depend on $\delta_{0}$. For arbitrary $\delta^{\prime}$ and $\delta^{\prime \prime}$ with $0<\delta^{\prime} \leqslant \delta^{\prime \prime}$, the natural inclusion $L_{(2)}^{n, q}(F)_{\varepsilon, \delta^{\prime}} \rightarrow L_{(2)}^{n, q}(F)_{\varepsilon, \delta^{\prime \prime}}$ is a bounded operator (continuous linear map) by $\|\bullet\|_{\varepsilon, \delta^{\prime \prime}} \leqslant\|\bullet\|_{\varepsilon, \delta^{\prime}}$ (see Lemma 2.2). By Lemma 2.12, the $F$-valued form $u_{\varepsilon, \delta_{\nu}}$ weakly converges to $\alpha_{\varepsilon, \delta^{\prime}}$ in not only $L_{(2)}^{n, q}(F)_{\varepsilon, \delta^{\prime}}$ but also $L_{(2)}^{n, q}(F)_{\varepsilon, \delta^{\prime \prime}}$. Hence it follows from the uniqueness of weak limits that $\alpha_{\varepsilon, \delta^{\prime}}=\alpha_{\varepsilon, \delta^{\prime \prime}}$. 


\section{INJECTIVITY THEOREMS FOR HIGHER DIRECT IMAGES}

We finally prove the estimate in the proposition. It is easy to see that

$$
\left\|\alpha_{\varepsilon}\right\|_{\varepsilon, \delta_{0}} \leqslant \lim _{\delta_{\nu} \rightarrow 0}\left\|u_{\varepsilon, \delta_{\nu}}\right\|_{\varepsilon, \delta_{0}} \leqslant \varliminf_{\delta_{\nu} \rightarrow 0}\left\|u_{\varepsilon, \delta_{\nu}}\right\|_{\varepsilon, \delta_{\nu}} \leqslant\|u\|_{H, \omega} .
$$

The first inequality follows since the norm is lower semi-continuous with respect to the weak convergence. The second inequality follows from $\omega_{\varepsilon, \delta_{0}} \geqslant \omega_{\varepsilon, \delta_{\nu}}$. The last inequality follows from inequality (3.6). Fatou's lemma yields

$$
\left\|\alpha_{\varepsilon}\right\|_{H_{\varepsilon}, \omega}^{2}=\int_{Y_{\varepsilon}}\left|\alpha_{\varepsilon}\right|_{H_{\varepsilon}, \omega}^{2} d V_{\omega} \leqslant \varliminf_{\delta_{0} \rightarrow 0} \int_{Y_{\varepsilon}}\left|\alpha_{\varepsilon}\right|_{H_{\varepsilon}, \omega_{\varepsilon, \delta_{0}}}^{2} d V_{\omega_{\varepsilon, \delta_{0}}}=\varliminf_{\delta_{0} \rightarrow 0}^{\lim }\left\|\alpha_{\varepsilon}\right\|_{\varepsilon, \delta_{0}}^{2} .
$$

These inequalities lead to the desired estimate.

For simplicity, we use the same notation $u_{\varepsilon, \delta}$ for the subsequence $u_{\varepsilon, \delta_{\nu}}$ in Proposition 3.4. We now consider a suitable limit of the $\alpha_{\varepsilon}$. For a fixed positive number $\varepsilon_{0}>0$, by taking a sufficiently small $\varepsilon$, we have

$$
\left\|\alpha_{\varepsilon}\right\|_{H_{\varepsilon_{0}}, \omega} \leqslant\left\|\alpha_{\varepsilon}\right\|_{H_{\varepsilon}, \omega} \leqslant\|u\|_{H, \omega}
$$

from property (b) and Proposition 3.4. By taking a subsequence of $\left\{\alpha_{\varepsilon}\right\}_{\varepsilon>0}$, we may assume that $\left\{\alpha_{\varepsilon}\right\}_{\varepsilon>0}$ weakly converges to some $\alpha$ in $L_{(2)}^{n, q}(F)_{H_{\varepsilon_{0}}, \omega}$. The following proposition reduces the proof of Theorem 1.2 to showing that the weak limit $\alpha$ is zero.

Proposition 3.6. If the weak limit $\alpha$ is zero in $L_{(2)}^{n, q}(F)_{H_{\varepsilon_{0}}, \omega}$, then the cohomology class $A$ is zero in $H^{q}\left(X, K_{X} \otimes F \otimes \mathcal{I}(h)\right)$.

Proof. We first consider the de Rham-Weil isomorphism constructed in Proposition 2.16:

$$
\frac{\operatorname{Ker} \bar{\partial}}{\operatorname{Im} \bar{\partial}} \text { of } L_{(2, \text { loc })}^{n, q}(F)_{\varepsilon, \delta_{0}} \underset{\phi_{1}}{\stackrel{\cong}{\longrightarrow}} \check{H}^{q}\left(X, K_{X} \otimes F \otimes \mathcal{I}\left(h_{\varepsilon}\right)\right)=\check{H}^{q}\left(X, K_{X} \otimes F \otimes \mathcal{I}(h)\right) .
$$

The Čech cohomology group does not depend on $\varepsilon$ by property (c), and the subspace $\operatorname{Im} \bar{\partial}$ is closed in $L_{(2, \mathrm{loc})}^{n, q}(F)_{\varepsilon, \delta_{0}}$ by Proposition 2.18. Hence, for every $\delta$ with $0<\delta \leqslant \delta_{0}$, we obtain

$$
\begin{aligned}
u-u_{\varepsilon, \delta} \in \overline{\operatorname{Im} \bar{\partial}} \text { in } L_{(2)}^{n, q}(F)_{\varepsilon, \delta} & \subset \overline{\operatorname{Im} \bar{\partial}} \text { in } L_{(2)}^{n, q}(F)_{\varepsilon, \delta_{0}} \\
& \subset \overline{\operatorname{Im} \bar{\partial}} \text { in } L_{(2, \text { loc })}^{n, q}(F)_{\varepsilon, \delta_{0}}=\operatorname{Im} \bar{\partial} \text { in } L_{(2, \text { loc })}^{n, q}(F)_{\varepsilon, \delta_{0}}
\end{aligned}
$$

from the construction of $u_{\varepsilon, \delta}$ and Proposition 2.18. As $\delta \rightarrow 0$, we obtain

$$
u-\alpha_{\varepsilon} \in \overline{\operatorname{Im} \bar{\partial}} \text { in } L_{(2)}^{n, q}(F)_{\varepsilon, \delta_{0}} \subset \overline{\operatorname{Im} \bar{\partial}} \text { in } L_{(2, \text { loc })}^{n, q}(F)_{\varepsilon, \delta_{0}}=\operatorname{Im} \bar{\partial} \text { in } L_{(2, \text { loc })}^{n, q}(F)_{\varepsilon, \delta_{0}}
$$

from Lemma 2.11 and Proposition 3.4. On the other hand, we have the following commutative diagram:

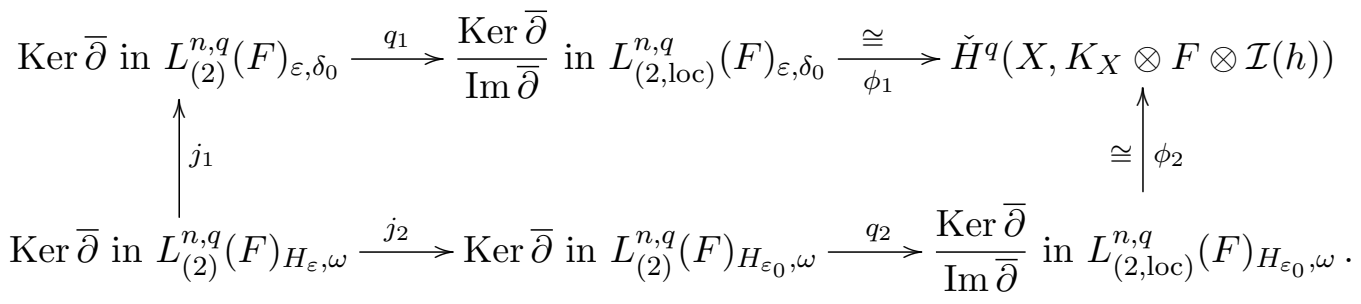

Here $j_{1}$ and $j_{2}$ are the natural inclusions, $q_{1}$ and $q_{2}$ are the natural quotient maps via the local $L^{2}$ spaces, and $\phi_{1}$ and $\phi_{2}$ are the de Rham-Weil isomorphisms. Note that $j_{2}$ is well defined. Indeed, by the $L^{2}$-integrability and [Dem82, Lemme 6.9], the equality $\bar{\partial} U=0$ can be extended from $Y_{\varepsilon}$ 


\section{S. Matsumura}

to $X$ (in particular to $Y_{\varepsilon_{0}}$ ) for $U \in \operatorname{Ker} \bar{\partial} \subset L_{(2)}^{n, q}(F)_{H_{\varepsilon}, \omega}$. Here we used the $L^{2}$-integrability with respect to $\omega\left(\right.$ not $\left.\omega_{\varepsilon, \delta}\right)$. From Proposition 2.19, the map $q_{2}$ is a compact operator; hence we obtain

$$
\lim _{\varepsilon \rightarrow 0} q_{2}\left(u-\alpha_{\varepsilon}\right)=q_{2}(u-\alpha)=q_{2}(u)
$$

from Lemma 2.13 and the assumption $\alpha=0$. On the other hand, we can see that $q_{1}\left(u-\alpha_{\varepsilon}\right)=0$ by the first half argument. Therefore, we obtain $q_{2}(u)=0$ from the above commutative diagram. Then, it follows from the following commutative diagram that $q_{3}(u)=0$, which indicates that $u$ belongs to $\operatorname{Im} \bar{\partial}$ in $L_{(2, \text { loc })}^{n, q}(F)_{H, \omega}$ :

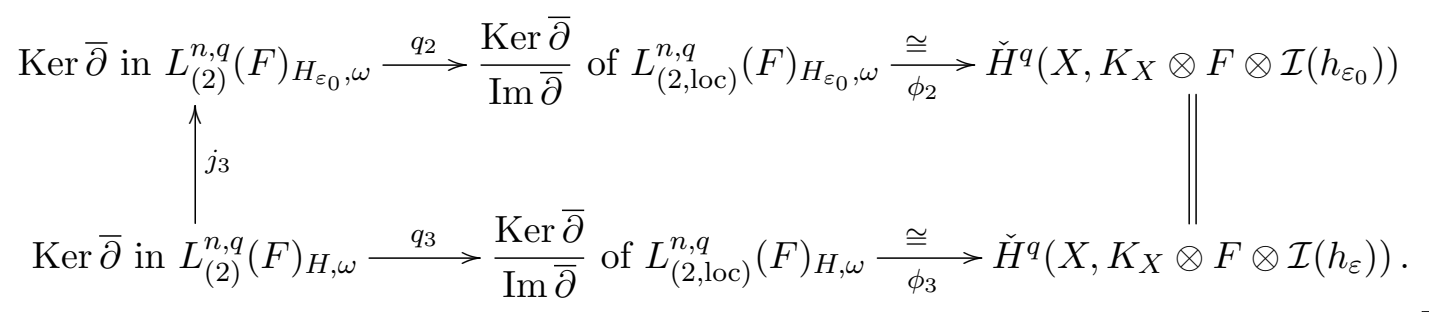

Step 3 (Relations between weak limits and $\boldsymbol{L}^{\mathbf{2}}$-norms). In this step, we consider the norm

$$
\left\|s u_{\varepsilon, \delta}\right\|_{\varepsilon, \delta}:=\left\|s u_{\varepsilon, \delta}\right\|_{H_{\varepsilon} h_{\varepsilon}^{m}, \omega_{\varepsilon, \delta}}
$$

and prove Proposition 3.7, which reduces the proof to showing that

$$
\varliminf_{\varepsilon \rightarrow 0} \varliminf_{\delta \rightarrow 0}\left\|s u_{\varepsilon, \delta}\right\|_{K, \varepsilon, \delta}=0
$$

for every relatively compact set $K \Subset X$.

To clarify a relation between the weak limit $\alpha$ and the asymptotic behavior of the norm of $s u_{\varepsilon, \delta}$, we compare the norm of $u_{\varepsilon, \delta}$ to the norm of $s u_{\varepsilon, \delta}$. We define $Y_{\varepsilon_{0}}^{k}$ and $X_{c}$ by

$$
Y_{\varepsilon_{0}}^{k}:=\left\{\left.y \in Y_{\varepsilon_{0}}|| s\right|_{h_{\varepsilon_{0}}^{m}}(y)>1 / k\right\} \quad \text { and } \quad X_{c}:=\{x \in X \mid \Phi(x)<c\}
$$

for $k \gg 0$ and $c$. The subset $X_{c}$ is a relatively compact set in $X$ for every $c$ with $c<\sup _{X} \Phi$ by the construction of $\Phi$. Furthermore, the set $Y_{\varepsilon_{0}}^{k}$ is an open set in $Y_{\varepsilon_{0}}$ since $|s|_{h_{\varepsilon_{0}}^{m}}$ is lower semi-continuous. Then, we prove the following proposition.

Proposition 3.7. In the above situation, if we have

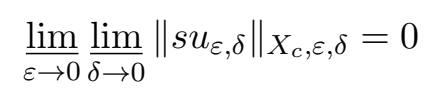

for every $c$ with $c<\sup _{X} \Phi$, then the weak limit $\alpha$ is zero. In particular, the cohomology class $A$ is zero by Proposition 3.6.

Proof. By the argument of inequality (3.8), we are assuming that $\alpha_{\varepsilon}$ weakly converges to $\alpha$ in $L_{(2)}^{n, q}(F)_{H_{\varepsilon_{0}}, \omega}$. The restriction $\left.\alpha_{\varepsilon}\right|_{X_{c} \cap Y_{\varepsilon_{0}}^{k}}$ also weakly converges to $\left.\alpha\right|_{X_{c} \cap Y_{\varepsilon_{0}}^{k}}$ in $L_{(2)}^{n, q}\left(X_{c} \cap Y_{\varepsilon_{0}}^{k}, F\right)_{H_{\varepsilon_{0}}, \omega}$ by Lemma 2.12 since the restriction map

$$
L_{(2)}^{n, q}(F)_{H_{\varepsilon_{0}}, \omega} \longrightarrow L_{(2)}^{n, q}\left(X_{c} \cap Y_{\varepsilon_{0}}^{k}, F\right)_{H_{\varepsilon_{0}, \omega}}
$$

is a bounded operator (continuous linear map). Therefore, we obtain

$$
\|\alpha\|_{X_{c} \cap Y_{\varepsilon_{0}}^{k}, H_{\varepsilon_{0}}, \omega} \leqslant \underline{\lim _{\varepsilon \rightarrow 0}}\left\|\alpha_{\varepsilon}\right\|_{X_{c} \cap Y_{\varepsilon_{0}}^{k}, H_{\varepsilon_{0}}, \omega} \leqslant \underline{\underline{\lim }}\left\|\alpha_{\varepsilon}\right\|_{X_{c} \cap Y_{\varepsilon_{0}}^{k}, H_{\varepsilon}, \omega} .
$$




\section{INJECTIVITY THEOREMS FOR HIGHER DIRECT IMAGES}

The first inequality follows since the norm is lower semi-continuous with respect to the weak convergence, and the second inequality follows from property (b). By the same argument, the restriction of $u_{\varepsilon, \delta}$ weakly converges to $\alpha_{\varepsilon}$ in $L_{(2)}^{n, q}\left(X_{c} \cap Y_{\varepsilon_{0}}^{k}, F\right)_{\varepsilon, \delta_{0}}$, and thus we obtain

$$
\left\|\alpha_{\varepsilon}\right\|_{X_{c} \cap Y_{\varepsilon_{0}}^{k}, \varepsilon, \delta_{0}} \leqslant \underline{\lim _{\delta \rightarrow 0}}\left\|u_{\varepsilon, \delta}\right\|_{X_{c} \cap Y_{\varepsilon_{0}}^{k}, \varepsilon, \delta_{0}} \leqslant \varliminf_{\delta \rightarrow 0}\left\|u_{\varepsilon, \delta}\right\|_{X_{c} \cap Y_{\varepsilon_{0}}^{k}, \varepsilon, \delta} .
$$

Moreover, we can obtain

$$
\left\|\alpha_{\varepsilon}\right\|_{X_{c} \cap Y_{\varepsilon_{0}}^{k}, H_{\varepsilon}, \omega} \leqslant \varliminf_{\delta_{0} \rightarrow 0}\left\|\alpha_{\varepsilon}\right\|_{X_{c} \cap Y_{\varepsilon_{0}}^{k}, \varepsilon, \delta_{0}} \leqslant \frac{\lim }{\delta \rightarrow 0}\left\|u_{\varepsilon, \delta}\right\|_{X_{c} \cap Y_{\varepsilon_{0}}^{k}, \varepsilon, \delta}
$$

by the above inequality and Fatou's lemma (see the argument for inequality (3.7)). These inequalities yield

$$
\|\alpha\|_{X_{c} \cap Y_{\varepsilon_{0}}^{k}, H_{\varepsilon_{0}, \omega}} \leqslant \underline{\lim _{\varepsilon \rightarrow 0}} \frac{\lim }{\delta \rightarrow 0}\left\|u_{\varepsilon, \delta}\right\|_{X_{c} \cap Y_{\varepsilon_{0}}^{k}, \varepsilon, \delta} .
$$

On the other hand, from $1 / k<|s|_{h_{\varepsilon_{0}}^{m}} \leqslant|s|_{h_{\varepsilon}^{m}}$ on $Y_{\varepsilon_{0}}^{k}$, we obtain

$$
\left\|u_{\varepsilon, \delta}\right\|_{X_{c} \cap Y_{\varepsilon_{0}}^{k}, \varepsilon, \delta} \leqslant k\left\|s u_{\varepsilon, \delta}\right\|_{X_{c} \cap Y_{\varepsilon_{0}}^{k}, \varepsilon, \delta} \leqslant k\left\|s u_{\varepsilon, \delta}\right\|_{X_{c}, \varepsilon, \delta} .
$$

By assumption, we can conclude that $\alpha=0$ on $X_{c} \cap Y_{\varepsilon_{0}}^{k}$ for arbitrary $c<\sup \Phi$ and $k \gg 0$. From $\cup_{\text {sup } \Phi>c, k \gg 0}\left(X_{c} \cap Y_{\varepsilon_{0}}^{k}\right)=Y_{\varepsilon_{0}}$, we obtain the conclusion.

Step 4 (Construction of solutions of the $\bar{\partial}$-equation). In this step, using the construction of the de Rham-Weil isomorphism in Subsection 2.6, we prove Proposition 3.9, which gives a solution $w_{\varepsilon, \delta}$ of the $\bar{\partial}$-equation $\bar{\partial} w_{\varepsilon, \delta}=u-u_{\varepsilon, \delta}$ with uniformly bounded (local) $L^{2}$-norm.

Fix a locally finite open cover $\mathcal{U}:=\left\{B_{i}\right\}_{i \in I}$ of $X$ by sufficiently small Stein open sets $B_{i} \Subset X$. Since $h_{\varepsilon}, \omega_{\varepsilon, \delta}$, and $Y_{\varepsilon}$ satisfy the assumptions in Proposition 2.16, we have the continuous maps

$$
\begin{aligned}
& f_{\varepsilon, \delta}: \operatorname{Ker} \bar{\partial} \text { in } L_{(2, \text { loc })}^{n, q}(F)_{\varepsilon, \delta} \rightarrow \operatorname{Ker} \mu \text { in } C^{q}\left(\mathcal{U}, K_{X} \otimes F \otimes \mathcal{I}\left(h_{\varepsilon}\right)\right), \\
& g_{\varepsilon, \delta}: \operatorname{Ker} \mu \text { in } C^{q}\left(\mathcal{U}, K_{X} \otimes F \otimes \mathcal{I}\left(h_{\varepsilon}\right)\right) \rightarrow \operatorname{Ker} \bar{\partial} \text { in } L_{(2, \text { loc })}^{n, q}(F)_{\varepsilon, \delta}
\end{aligned}
$$

that determine the isomorphism between the $\bar{\partial}$-cohomology and the Cech cohomology. For the construction of $f_{\varepsilon, \delta}$ in Proposition 2.16, we locally solved the $\bar{\partial}$-equation and estimated the $L^{2}$ norm of the solution by Lemma 2.15. In this subsection, for the $L^{2}$-estimate of the solution, we use the following lemma instead of Lemma 2.15.

Lemma 3.8. Let $B \Subset X$ be a sufficiently small Stein open set. Then, for an arbitrary $U \in \operatorname{Ker} \bar{\partial} \subset$ $L_{(2)}^{n, q}\left(B \backslash Z_{\varepsilon}, F\right)_{\varepsilon, \delta}$, there exist $V \in L_{(2)}^{n, q-1}\left(B \backslash Z_{\varepsilon}, F\right)_{\varepsilon, \delta}$ and a positive constant $C_{\varepsilon, \delta}$ (depending only on $\left.\Psi_{\varepsilon, \delta}, q\right)$ such that

- we have $\bar{\partial} V=U$ and $\|V\|_{\varepsilon, \delta} \leqslant C_{\varepsilon, \delta}\|U\|_{\varepsilon, \delta}$;

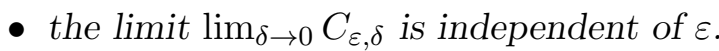

Proof. We may assume $\varepsilon<1 / 2$. Furthermore, by property (C), we may assume that there exists a bounded function $\Psi_{\varepsilon, \delta}$ on $B$ such that $\omega_{\varepsilon, \delta}=\sqrt{-1} \partial \bar{\partial} \Psi_{\varepsilon, \delta}$ and $\Psi_{\varepsilon, \delta} \rightarrow \Psi$ as $\delta \rightarrow 0$. The curvature of $G_{\varepsilon, \delta}$ defined by $G_{\varepsilon, \delta}:=H_{\varepsilon} e^{-\Psi_{\varepsilon, \delta}}$ satisfies

$$
\begin{aligned}
\sqrt{-1} \Theta_{G_{\varepsilon, \delta}}(F) & =\sqrt{-1} \Theta_{h_{\varepsilon}}(F)+\sqrt{-1} \partial \bar{\partial} \chi \circ \Phi+\sqrt{-1} \partial \bar{\partial} \Psi_{\varepsilon, \delta} \\
& \geqslant-\varepsilon \omega+\omega_{\varepsilon, \delta} \\
& \geqslant(1-\varepsilon) \omega_{\varepsilon, \delta}
\end{aligned}
$$




\section{S. Matsumura}

by property (a) and property (B). Here we used the inequality $\sqrt{-1} \partial \bar{\partial} \chi \circ \Phi \geqslant 0$, which follows since $\Phi$ is a psh function and $\chi$ is an increasing convex function. We can see that $\|U\|_{G_{\varepsilon, \delta}, \omega_{\varepsilon, \delta}}$ is finite since $\Psi_{\varepsilon, \delta}$ is a bounded function. Hence, by the standard result for the $\bar{\partial}$-equation, there exists a solution $V$ such that

$$
\bar{\partial} V=U \quad \text { and } \quad\|V\|_{G_{\varepsilon, \delta}, \omega_{\varepsilon, \delta}}^{2} \leqslant \frac{1}{q(1-\varepsilon)}\|U\|_{G_{\varepsilon, \delta}, \omega_{\varepsilon, \delta}}^{2} .
$$

From $(1-\varepsilon)>1 / 2$ and the definition of $G_{\varepsilon, \delta}$, we can easily see that

$$
\|V\|_{\varepsilon, \delta} \leqslant \sqrt{\frac{2}{q}} \frac{\sup _{B} e^{-\Psi_{\varepsilon, \delta}}}{\inf _{B} e^{-\Psi_{\varepsilon, \delta}}}\|U\|_{\varepsilon, \delta} .
$$

The constant on the right-hand side converges to $(2 / q)^{1 / 2}\left(\sup _{B} e^{-\Psi} / \inf _{B} e^{-\Psi}\right)$ as $\delta \rightarrow 0$.

Proposition 3.9. For every $c$ with $c<\sup _{X} \Phi$, there exists a $w_{\varepsilon, \delta} \in L_{(2, \text { loc })}^{n, q-1}(F)_{\varepsilon, \delta}$ with the following properties:

- We have $\bar{\partial} w_{\varepsilon, \delta}=u-u_{\varepsilon, \delta}$.

- The limit $\varlimsup_{\delta \rightarrow 0}\left\|w_{\varepsilon, \delta}\right\|_{X_{c}, \varepsilon, \delta}$ can be bounded by a constant independent of $\varepsilon$.

Remark 3.10. We already know that there exists a solution $w_{\varepsilon, \delta}$ of the $\bar{\partial}$-equation $\bar{\partial} w_{\varepsilon, \delta}=u-u_{\varepsilon, \delta}$ by $u-u_{\varepsilon, \delta} \in \operatorname{Im} \bar{\partial} \subset L_{(2, \text { loc })}^{n, q}(F)_{\varepsilon, \delta}$ (see (3.9) in the proof of Proposition 3.6). The important point here is the second property on the local $L^{2}$-norm of solutions.

The strategy of the proof is the same as in the proof of [Mat18a, Theorem 5.9]. The main idea is to change the $\bar{\partial}$-equation $\bar{\partial} w_{\varepsilon, \delta}=u-u_{\varepsilon, \delta}$ to the equation $\mu \gamma_{\varepsilon, \delta}=f_{\varepsilon, \delta}\left(u-u_{\varepsilon, \delta}\right)$ of the coboundary operator $\mu$ in the set of cochains $C^{\bullet}\left(K_{X} \otimes F \otimes \mathcal{I}\left(h_{\varepsilon}\right)\right)$, by using the Cech complex and pursuing the de Rham-Weil isomorphism. Here $f_{\varepsilon, \delta}$ is the continuous map constructed in Proposition 2.16. The $L^{2}$-space $L_{(2)}^{n, q}(F)_{\varepsilon, \delta}$ depends on $\varepsilon$ and $\delta$, but $C^{\bullet}\left(K_{X} \otimes F \otimes \mathcal{I}\left(h_{\varepsilon}\right)\right)$ does not depend on them by property (c). This is one of the important points. In the proof, we will show that $f_{\varepsilon, \delta}\left(u-u_{\varepsilon, \delta}\right)$ converges to some $q$-coboundary $\alpha_{0,0}$ in $C^{q}\left(K_{X} \otimes F \otimes \mathcal{I}(h)\right)$ with respect to the topology induced by the local $L^{2}$-norms $\left\{p_{K_{i_{0} \ldots i_{q}}}(\bullet)\right\}_{K_{i_{0} \ldots i_{q}} \Subset B_{i_{0} \ldots i_{q}}}$ (see (2.2) for the definition). Furthermore, we will show that the coboundary operator $\mu$ is an open map. Then, by these observations, we will construct a solution $\gamma_{\varepsilon, \delta}$ of the equation $\mu \gamma_{\varepsilon, \delta}=f_{\varepsilon, \delta}\left(u-u_{\varepsilon, \delta}\right)$ with suitable local $L^{2}$-norm. Finally, by using the continuous map $g_{\varepsilon, \delta}$ constructed by a partition of unity, we conversely construct $w_{\varepsilon, \delta}$ satisfying the properties in Proposition 3.9.

For the reader's convenience, we first give a proof for the case $q=1$. This case helps us to understand the essential arguments.

Proof of Proposition 3.9 for the case $q=1$. We may assume that the cardinality of $I_{c}$ defined by

$$
I_{c}:=\left\{i \in I \mid B_{i} \cap X_{c} \neq \emptyset\right\}
$$

is finite by $X_{c} \Subset X$. For simplicity, we set $U_{\varepsilon, \delta}:=u-u_{\varepsilon, \delta}$. By Lemma 3.8, we can take a solution $\beta_{\varepsilon, \delta, i}$ of the $\bar{\partial}$-equation $\bar{\partial} \beta_{\varepsilon, \delta, i}=U_{\varepsilon, \delta}$ on $B_{i} \backslash Z_{\varepsilon}$ such that

$$
\left\|\beta_{\varepsilon, \delta, i}\right\|_{B_{i}, \varepsilon, \delta} \leqslant C_{\varepsilon, \delta}\left\|U_{\varepsilon, \delta}\right\|_{B_{i}, \varepsilon, \delta} \leqslant C_{\varepsilon, \delta}\left\|U_{\varepsilon, \delta}\right\|_{\varepsilon, \delta}
$$

for some constant $C_{\varepsilon, \delta}$. In the proof, the notation $C_{\varepsilon, \delta}$ denotes a (possibly different) positive constant with the property in Lemma 3.8 (that is, $\lim _{\delta \rightarrow 0} C_{\varepsilon, \delta}$ is independent of $\varepsilon$ ). Inequality (3.6) yields

$$
\left\|U_{\varepsilon, \delta}\right\|_{\varepsilon, \delta} \leqslant\|u\|_{\varepsilon, \delta}+\left\|u_{\varepsilon, \delta}\right\|_{\varepsilon, \delta} \leqslant 2\|u\|_{H, \omega}
$$




\section{INJECTIVITY THEOREMS FOR HIGHER DIRECT IMAGES}

In particular, the norm $\left\|\beta_{\varepsilon, \delta, i}\right\|_{B_{i}, \varepsilon, \delta}$ can be bounded by a constant $C_{\varepsilon, \delta}$.

We now consider the $F$-valued $(n, 0)$-form $\left(\beta_{\varepsilon, \delta, j}-\beta_{\varepsilon, \delta, i}\right)$ on $B_{i j} \backslash Z_{\varepsilon}$, where $B_{i j}:=B_{i} \cap B_{j}$. Then $\left(\beta_{\varepsilon, \delta, j}-\beta_{\varepsilon, \delta, i}\right)$ can be seen as a holomorphic function with bounded $L^{2}$-norm since it is a $\bar{\partial}$-closed $F$-valued $(n, 0)$-form satisfying $\left\|\beta_{\varepsilon, \delta, j}-\beta_{\varepsilon, \delta, i}\right\|_{H_{\varepsilon}, \omega}=\left\|\beta_{\varepsilon, \delta, j}-\beta_{\varepsilon, \delta, i}\right\|_{\varepsilon, \delta}<\infty$ (see Lemma 2.2). By the Riemann extension theorem, it can be extended to the $F$-valued $(n, 0)$-form on $B_{i j}$ (for which we use the same notation). Furthermore, it belongs to $H^{0}\left(B_{i j}, K_{X} \otimes F \otimes \mathcal{I}(h)\right)$ by property (c). Note that we can use property (c) thanks to a special property of $(n, 0)$-forms (holomorphic functions).

We define the 1-cocycle $\alpha_{\varepsilon, \delta}$ by

$$
\alpha_{\varepsilon, \delta}:=\mu\left\{\beta_{\varepsilon, \delta, i}\right\}=\left\{\beta_{\varepsilon, \delta, j}-\beta_{\varepsilon, \delta, i}\right\},
$$

where $\mu$ is the coboundary operator. The topology of $C^{q}\left(\mathcal{U}, K_{X} \otimes F \otimes \mathcal{I}(h)\right)$ is induced by the semi-norms $\left\{p_{K}(\bullet)\right\}_{K \Subset B_{i_{0} \ldots i_{q}}}$ defined to be

$$
p_{K}^{2}\left(\left\{\alpha_{i_{0} \ldots i_{q}}\right\}\right):=\int_{K}\left|\alpha_{i_{0} \ldots i_{q}}\right|_{H, \omega}^{2} d V_{\omega}
$$

for every $\left\{\alpha_{i_{0} \ldots i_{q}}\right\} \in C^{q}\left(\mathcal{U}, K_{X} \otimes F \otimes \mathcal{I}(h)\right)$ and $K \Subset U_{i_{0} \ldots i_{q}}$. The above integrand is independent of $\omega$ since $\alpha_{i_{0} \ldots i_{q}}$ is an $F$-valued $(n, 0)$-form (see Lemma 2.2). Then $C^{p}\left(\mathcal{U}, K_{X} \otimes F \otimes \mathcal{I}(h)\right.$ ) is a Fréchet space with respect to these semi-norms by Lemma 2.14. We prove the following claim.

Claim 3.11. There exist subsequences $\left\{\varepsilon_{k}\right\}_{k=1}^{\infty}$ and $\left\{\delta_{\ell}\right\}_{\ell=1}^{\infty}$ with the following properties:

- We have $\alpha_{\varepsilon_{k}, \delta_{\ell}} \rightarrow \alpha_{\varepsilon_{k}, 0}$ in $C^{1}\left(\mathcal{U}, K_{X} \otimes F \otimes \mathcal{I}(h)\right)$ as $\delta_{\ell} \rightarrow 0$.

- We have $\alpha_{\varepsilon_{k}, 0} \rightarrow \alpha_{0,0}$ in $C^{1}\left(\mathcal{U}, K_{X} \otimes F \otimes \mathcal{I}(h)\right)$ as $\varepsilon_{\ell} \rightarrow 0$.

Proof of Claim 3.11. We regard $\alpha_{\varepsilon, \delta, i j}:=\beta_{\varepsilon, \delta, j}-\beta_{\varepsilon, \delta, i}$ as a holomorphic function on $B_{i j}$. By the construction of $\beta_{\varepsilon, \delta, i}$, the norm $\left\|\alpha_{\varepsilon, \delta, i j}\right\|_{B_{i j}, \varepsilon, \delta}$ can be bounded by a constant $C_{\varepsilon, \delta}$. This indicates that the sup-norm $\sup _{K}\left|\alpha_{\varepsilon, \delta, i j}\right|$ is also uniformly bounded with respect to $\delta$ for every $K \Subset B_{i j}$. (Recall the local sup-norm of holomorphic functions can be estimated by the $L^{2}$-norm). By Montel's theorem, we can take a subsequence $\left\{\delta_{\ell}\right\}_{\ell=1}^{\infty}$ with the first property. Then, the norm of the limit $\alpha_{\varepsilon, 0}$ can be bounded by a positive constant independent of $\varepsilon$ since $\lim _{\delta \rightarrow 0} C_{\varepsilon, \delta}$ is independent of $\varepsilon$. Thus, we can take a subsequence $\left\{\varepsilon_{k}\right\}_{k=1}^{\infty}$ with the second properties. The convergence with respect to the local sup-norms implies the convergence with respect to the local $L^{2}$-norms $\left\{p_{K}(\bullet)\right\}_{K \Subset B_{i_{0} \ldots i_{q}}}$ (see, for example, [Mat18a, Lemma 5.2]). This completes the proof.

For simplicity, we continue to use the same notation for the subsequence in Claim 3.11.

Claim 3.12. The cocycle $\alpha_{\varepsilon, \delta}$ is a coboundary. In particular, the limit $\alpha_{0,0}$ is also a coboundary.

Proof of Claim 3.12. By Remark 3.10, we can see that $U_{\varepsilon, \delta}=u-u_{\varepsilon, \delta}$ belongs to $\operatorname{Im} \bar{\partial}$ in $L_{(2, \text { loc })}^{n, q}(F)_{\varepsilon, \delta}$, and, by the isomorphism in Proposition 2.16, we can see that $\alpha_{\varepsilon, \delta}$ is a coboundary. Since we are assuming that $X$ is holomorphically convex (see Step 1), the set of $q$-coboundaries $B^{q}\left(\mathcal{U}, K_{X} \otimes F \otimes \mathcal{I}(h)\right)=\operatorname{Im} \mu$ is a Fréchet space by Lemma 2.14; hence we obtain the latter conclusion.

Proof of Proposition 3.9 for the case $q=1$, continued. We will construct a solution $\gamma_{\varepsilon, \delta}$ of the $\mu$-equation $\mu \gamma_{\varepsilon, \delta}=\alpha_{\varepsilon, \delta}$ with suitable local $L^{2}$-norm. The coboundary operator

$$
\mu: C^{q-1}\left(\mathcal{U}, K_{X} \otimes F \otimes \mathcal{I}(h)\right) \rightarrow B^{q}\left(\mathcal{U}, K_{X} \otimes F \otimes \mathcal{I}(h)\right)
$$




\section{S. Matsumura}

is continuous and surjective between Fréchet spaces, and thus, it is an open map by the open mapping theorem. From the latter conclusion of Claim 3.12, there exists a $\gamma_{0,0} \in C^{0}\left(\mathcal{U}, K_{X} \otimes\right.$ $F \otimes \mathcal{I}(h))$ such that $\mu \gamma_{0,0}=\alpha_{0,0}$. For an arbitrary family $K:=\left\{K_{i}\right\}_{i \in I_{c}}$ of relative compact sets $K_{i} \Subset B_{i}$, the image $\mu\left(\Delta_{K}\right)$ of $\Delta_{K}$ is an open neighborhood of $\alpha_{0,0}$, where $\Delta_{K}$ is an open neighborhood of $\gamma_{0,0}$ defined by

$$
\Delta_{K}:=\left\{\gamma \in C^{0}\left(\mathcal{U}, K_{X} \otimes F \otimes \mathcal{I}(h)\right) \mid p_{K_{i}}\left(\gamma-\gamma_{0,0}\right)<1 \text { for every } i \in I_{c}\right\} .
$$

Since the image $\mu\left(\Delta_{K}\right)$ is an open neighborhood of $\alpha_{0,0}$ and $\alpha_{\varepsilon, \delta}$ converges to $\alpha_{0,0}$, we can take $\gamma_{\varepsilon, \delta}=\left\{\gamma_{\varepsilon, \delta, i}\right\} \in \Delta_{K}$ such that

$$
\begin{aligned}
\left\{\gamma_{\varepsilon, \delta, j}-\gamma_{\varepsilon, \delta, i}\right\} & =\mu \gamma_{\varepsilon, \delta}=\alpha_{\varepsilon, \delta}=\left\{\beta_{\varepsilon, \delta, j}-\beta_{\varepsilon, \delta, i}\right\} \\
p_{K_{i}}^{2}\left(\gamma_{\varepsilon, \delta}\right) & =\int_{K_{i}}\left|\gamma_{\varepsilon, \delta, i}\right|_{H, \omega}^{2} d V_{\omega} \leqslant C_{K} \text { for every } i \in I_{c}
\end{aligned}
$$

for some positive constant $C_{K}$ (depending on $K$ and $\gamma_{0,0}$ but not on $\varepsilon$ and $\delta$ ).

Let us construct a solution $w_{\varepsilon, \delta}$ with the properties in Proposition 3.9. We fix a partition of unity $\left\{\rho_{i}\right\}_{i \in I}$. Then, from $\bar{\partial} \gamma_{\varepsilon, \delta, i}=0$ and $\bar{\partial} \beta_{\varepsilon, \delta, i}=U_{\varepsilon, \delta}$, we have

$$
\begin{aligned}
\bar{\partial} \sum_{k \in I} \rho_{k}\left(\gamma_{\varepsilon, \delta, i}-\gamma_{\varepsilon, \delta, k}\right) & =-\bar{\partial} \sum_{k \in I} \rho_{k} \gamma_{\varepsilon, \delta, k} \\
\bar{\partial} \sum_{k \in I} \rho_{k}\left(\beta_{\varepsilon, \delta, i}-\beta_{\varepsilon, \delta, k}\right) & =U_{\varepsilon, \delta}-\bar{\partial} \sum_{k \in I} \rho_{k} \beta_{\varepsilon, \delta, k} .
\end{aligned}
$$

When we define $w_{\varepsilon, \delta}$ by

$$
w_{\varepsilon, \delta}:=\sum_{k \in I} \rho_{k} \beta_{\varepsilon, \delta, k}-\sum_{k \in I} \rho_{k} \gamma_{\varepsilon, \delta, k},
$$

it is easy to check $U_{\varepsilon, \delta}=\bar{\partial} w_{\varepsilon, \delta}$ by equality (3.11). It remains to estimate the $L^{2}$-norm of $w_{\varepsilon, \delta}$. By putting $K_{i}:=\operatorname{Supp} \rho_{i}$, we may assume that the inequality

$$
p_{K_{i}}^{2}\left(\gamma_{\varepsilon, \delta}\right)=\int_{\operatorname{Supp} \rho_{i}}\left|\gamma_{\varepsilon, \delta, i}\right|_{H, \omega}^{2} d V_{\omega} \leqslant C_{K}
$$

holds for every $i \in I_{c}$ by inequality (3.12). Therefore we obtain

$$
\begin{aligned}
\left\|\sum_{k \in I} \rho_{k} \gamma_{\varepsilon, \delta, k}\right\|_{X_{c}, \varepsilon, \delta}^{2} & =\int_{X_{c}}\left|\sum_{k \in I} \rho_{k} \gamma_{\varepsilon, \delta, k}\right|_{H_{\varepsilon}, \omega}^{2} d V_{\omega} \\
& \leqslant \sum_{k \in I_{c}} \int_{B_{k} \cap \operatorname{Supp} \rho_{k}}\left|\gamma_{\varepsilon, \delta, k}\right|_{H_{\varepsilon}, \omega}^{2} d V_{\omega} \leqslant C_{K} \sharp I_{c} .
\end{aligned}
$$

Note that the cardinality of $I_{c}$ is finite by the choice of $\mathcal{U}$. Furthermore, we obtain

$$
\begin{aligned}
\left\|\sum_{k \in I} \rho_{k} \beta_{\varepsilon, \delta, k}\right\|_{X_{c}, \varepsilon, \delta}^{2} & =\int_{X_{c}}\left|\sum_{k \in I} \rho_{k} \beta_{\varepsilon, \delta, k}\right|_{\varepsilon, \delta}^{2} d V_{\omega_{\varepsilon, \delta}} \\
& \leqslant \sum_{k \in I_{c}} \int_{B_{k}}\left|\beta_{\varepsilon, \delta, k}\right|_{\varepsilon, \delta}^{2} d V_{\omega_{\varepsilon, \delta}} \leqslant C_{\varepsilon, \delta}^{2} \sharp I_{c}\|u\|_{H, \omega}
\end{aligned}
$$

for some $C_{\varepsilon, \delta}>0$ by the construction of $\beta_{\varepsilon, \delta, i}$. These inequalities lead to the desired estimate of $w_{\varepsilon, \delta}$. 


\section{INJECTIVITY THEOREMS FOR HIGHER DIRECT IMAGES}

Proof of Proposition 3.9 for the general case. For simplicity, we set $U_{\varepsilon, \delta}:=u-u_{\varepsilon, \delta} \in \operatorname{Im} \bar{\partial} \subset$ $L_{(2, \mathrm{loc})}^{n, q}(F)_{\varepsilon, \delta}$. Then, there exist $F$-valued $(n, q-k-1)$-forms $\beta_{i_{0} \ldots i_{k}}^{\varepsilon, \delta}$ on $B_{i_{0} \ldots i_{k}} \backslash Z_{\varepsilon}$ satisfying

$$
\left\{\begin{array}{c}
\bar{\partial}\left\{\beta_{i_{0}}^{\varepsilon, \delta}\right\}=\left\{\left.U_{\varepsilon, \delta}\right|_{B_{i_{0}} \backslash Z_{\varepsilon}}\right\}, \\
\bar{\partial}\left\{\beta_{i_{0} i_{1}}^{\varepsilon, \delta}\right\}=\mu\left\{\beta_{i_{0}}^{\varepsilon, \delta}\right\}, \\
\bar{\partial}\left\{\beta_{i_{0} i_{1} i_{2}}^{\varepsilon, \delta}\right\}=\mu\left\{\beta_{i_{0} i_{1}}^{\varepsilon, \delta}\right\}, \\
\vdots \\
\bar{\partial}\left\{\beta_{i_{0} \ldots i_{q-1}}^{\varepsilon, \delta}\right\}=\mu\left\{\beta_{i_{0} \ldots i_{q-2}}^{\varepsilon, \delta}\right\}, \\
f_{\varepsilon, \delta}\left(U_{\varepsilon, \delta}\right)=\mu\left\{\beta_{i_{0} \ldots i_{q-1}}^{\varepsilon, \delta}\right\} .
\end{array}\right.
$$

Here $\beta_{i_{0} \ldots i_{k}}^{\varepsilon, \delta}$ is the solution of the above equation whose norm is minimum among all the solutions (see the construction of $f$ in Proposition 2.16). For example, $\beta_{i_{0}}^{\varepsilon, \delta}$ is the solution of $\bar{\partial} \beta_{i_{0}}^{\varepsilon, \delta}=U_{\varepsilon, \delta}$ on $B_{i_{0}} \backslash Z_{\varepsilon}$ whose norm $\left\|\beta_{i_{0}}^{\varepsilon, \delta}\right\|_{\varepsilon, \delta}$ is minimum among all the solutions. In particular, we have

$$
\left\|\beta_{i_{0}}^{\varepsilon, \delta}\right\|_{\varepsilon, \delta}^{2} \leqslant C_{\varepsilon, \delta}\left\|U_{\varepsilon, \delta}\right\|_{B_{i_{0}}, \varepsilon, \delta}^{2} \leqslant C_{\varepsilon, \delta}\left\|U_{\varepsilon, \delta}\right\|_{\varepsilon, \delta}^{2}
$$

for some constant $C_{\varepsilon, \delta}$ by Lemma 3.8, where $C_{\varepsilon, \delta}$ is a constant such that $\lim _{\delta \rightarrow 0} C_{\varepsilon, \delta}$ is independent of $\varepsilon$. Similarly, $\beta_{i_{0} i_{1}}^{\varepsilon, \delta}$ is the solution of $\bar{\partial} \beta_{i_{0} i_{1}}^{\varepsilon, \delta}=\left(\beta_{i_{1}}^{\varepsilon, \delta}-\beta_{i_{0}}^{\varepsilon, \delta}\right)$ on $B_{i_{0} i_{1}} \backslash Z_{\varepsilon}$ and the norm $\left\|\beta_{i_{0} i_{1}}^{\varepsilon, \delta}\right\|_{\varepsilon, \delta}$ is minimum among all the solutions. In particular, we have $\left\|\beta_{i_{0} i_{1}}^{\varepsilon, \delta}\right\|_{\varepsilon, \delta} \leqslant$ $D_{\varepsilon, \delta}\left\|\left(\beta_{i_{1}}^{\varepsilon, \delta}-\beta_{i_{0}}^{\varepsilon, \delta}\right)\right\|_{\varepsilon, \delta}$ for some constant $D_{\varepsilon, \delta}$. It is easy to see that

$$
\left\|\beta_{i_{0} i_{1}}^{\varepsilon, \delta}\right\|_{\varepsilon, \delta} \leqslant D_{\varepsilon, \delta}\left\|\left(\beta_{i_{1}}^{\varepsilon, \delta}-\beta_{i_{0}}^{\varepsilon, \delta}\right)\right\|_{\varepsilon, \delta} \leqslant 2 C_{\varepsilon, \delta} D_{\varepsilon, \delta}\left\|U_{\varepsilon, \delta}\right\|_{\varepsilon, \delta} \leqslant 2 C_{\varepsilon, \delta} D_{\varepsilon, \delta}\|u\|_{H, \omega} .
$$

By repeating this process, we obtain

$$
\left\|\beta_{i_{0} \ldots i_{k}}^{\varepsilon, \delta}\right\|_{\varepsilon, \delta}^{2} \leqslant C_{\varepsilon, \delta}\|u\|_{H, \omega}^{2}
$$

for a constant $C_{\varepsilon, \delta}$ such that $\lim _{\delta \rightarrow 0} C_{\varepsilon, \delta}$ is independent of $\varepsilon$. Moreover, by property (c), we obtain

$$
\begin{aligned}
\alpha_{\varepsilon, \delta}:=f_{\varepsilon, \delta}\left(U_{\varepsilon, \delta}\right)=\mu\left\{\beta_{i_{0} \ldots i_{q-1}}^{\varepsilon, \delta}\right\} & \in C^{q}\left(\mathcal{U}, K_{X} \otimes F \otimes \mathcal{I}\left(h_{\varepsilon}\right)\right) \\
& =C^{q}\left(\mathcal{U}, K_{X} \otimes F \otimes \mathcal{I}(h)\right) .
\end{aligned}
$$

In the same way as in Claims 3.11 and 3.12, we obtain the following.

Claim 3.13. There exist subsequences $\left\{\varepsilon_{k}\right\}_{k=1}^{\infty}$ and $\left\{\delta_{\ell}\right\}_{\ell=1}^{\infty}$ with the following properties:

- We have $\alpha_{\varepsilon_{k}, \delta_{\ell}} \rightarrow \alpha_{\varepsilon_{k}, 0}$ in $C^{q}\left(\mathcal{U}, K_{X} \otimes F \otimes \mathcal{I}(h)\right)$ as $\delta_{\ell} \rightarrow 0$.

- We have $\alpha_{\varepsilon_{k}, 0} \rightarrow \alpha_{0,0}$ in $C^{q}\left(\mathcal{U}, K_{X} \otimes F \otimes \mathcal{I}(h)\right)$ as $\varepsilon_{k} \rightarrow 0$.

Moreover, the limit $\alpha_{0,0}$ belongs to $B^{q}\left(\mathcal{U}, K_{X} \otimes F \otimes \mathcal{I}(h)\right)=\operatorname{Im} \mu$.

By the last conclusion of the claim, there exists a $\gamma_{0,0} \in C^{q-1}\left(\mathcal{U}, K_{X} \otimes F \otimes \mathcal{I}(h)\right)$ such that $\mu \gamma_{0,0}=\alpha_{0,0}$. The coboundary operator

$$
\mu: C^{q-1}\left(\mathcal{U}, K_{X} \otimes F \otimes \mathcal{I}(h)\right) \rightarrow B^{q}\left(\mathcal{U}, K_{X} \otimes F \otimes \mathcal{I}(h)\right)=\operatorname{Im} \mu
$$

is an open map by the open mapping theorem. For an arbitrary family $K:=\left\{K_{i}\right\}_{i \in I_{c}}$ of relative compact sets $K_{i} \Subset B_{i}$, we define $\Delta_{K}$ by (3.10). Then, since $\mu\left(\Delta_{K}\right)$ is an open neighborhood of the limit $\alpha_{0,0}$ in $\operatorname{Im} \mu$, we can obtain $\gamma_{\varepsilon, \delta} \in C^{q-1}\left(\mathcal{U}, K_{X} \otimes F \otimes \mathcal{I}(h)\right)$ such that

$$
\mu \gamma_{\varepsilon, \delta}=\alpha_{\varepsilon, \delta} \quad \text { and } \quad p_{K_{i_{0} \ldots i_{q-1}}}\left(\gamma_{\varepsilon, \delta}\right)^{2} \leqslant C_{K}
$$




\section{S. Matsumura}

for some positive constant $C_{K}$. The above constant $C_{K}$ depends on the choice of $K$ and $\gamma_{0,0}$, but does not depend on $\varepsilon$ and $\delta$.

By the same argument as in [Mat18a, Claims 5.11 and 5.13], we can obtain $F$-valued $(n, q-1)$ forms $w_{\varepsilon, \delta}$ with the desired properties. The strategy is as follows: The inverse map $\overline{g_{\varepsilon, \delta}}$ of $\overline{f_{\varepsilon, \delta}}$ is explicitly constructed by using a partition of unity (see Proposition 2.16). It is easy to see that $g_{\varepsilon, \delta}\left(\mu \gamma_{\varepsilon, \delta}\right)=\bar{\partial} v_{\varepsilon, \delta}$ and $g_{\varepsilon, \delta}\left(\alpha_{\varepsilon, \delta}\right)=U_{\varepsilon, \delta}+\bar{\partial} \widetilde{v}_{\varepsilon, \delta}$ hold for some $v_{\varepsilon, \delta}$ and $\widetilde{v}_{\varepsilon, \delta}$ by the de RhamWeil isomorphism. In particular, we have $U_{\varepsilon, \delta}=\bar{\partial}\left(v_{\varepsilon, \delta}-\widetilde{v}_{\varepsilon, \delta}\right)$ by $\mu \gamma_{\varepsilon, \delta}=\alpha_{\varepsilon, \delta}$. The important point here is that we can explicitly compute $v_{\varepsilon, \delta}$ and $\widetilde{v}_{\varepsilon, \delta}$ by using the partition of unity, $\beta_{i_{0} \ldots i_{k}, \delta}$, and $\gamma_{\varepsilon, \delta}$. From this explicit expression, we obtain the $L^{2}$-estimate for $v_{\varepsilon, \delta}$ and $\widetilde{v}_{\varepsilon, \delta}$. (In the case $q=1$, we have already obtained the $L^{2}$-estimate.) See [Mat18a, Claims 5.11 and 5.13] for the precise argument.

We close this subsection with the following corollary.

Corollary 3.14. For every $c$ with $c<\sup _{X} \Phi$, there exist $v_{\varepsilon, \delta} \in L_{(2, \text { loc })}^{n, q-1}\left(F^{m+1}\right)_{\varepsilon, \delta}$ with the following properties:

- We have $\bar{\partial} v_{\varepsilon, \delta}=s u_{\varepsilon, \delta}$.

- The limit $\varlimsup_{\delta \rightarrow 0}\left\|v_{\varepsilon, \delta}\right\|_{X_{c}, \varepsilon, \delta}$ can be bounded by a constant independent of $\varepsilon$.

Proof. Take $w_{\varepsilon, \delta}$ with the properties in Proposition 3.9. On the other hand, we are assuming that the cohomology class $s A=\{s u\}$ is zero, and thus there exists a $w$ such that $\bar{\partial} w=s u$ and $\|w\|_{X_{c}, H h^{m}, \omega}<\infty$. Then, the $F$-valued $(n, q-1)$-form $v_{\varepsilon, \delta}$ defined by $v_{\varepsilon, \delta}:=w-s w_{\varepsilon, \delta}$ satisfies the desired properties by $\sup _{X}|s|_{h_{\varepsilon}^{m}} \leqslant \sup _{X}|s|_{h^{m}}<\infty$.

Step 5 (Asymptotics of norms of differential forms). In this step, for every $b$ with $b<\sup _{X} \Phi$, we show that

$$
\varliminf_{\varepsilon \rightarrow 0} \varliminf_{\delta \rightarrow 0}\left\|s u_{\varepsilon, \delta}\right\|_{X_{b}, \varepsilon, \delta}=0
$$

This completes the proof by Proposition 3.7. For every $b$ with $b<\sup _{X} \Phi$, there exists a $c$ such that $b<c<\sup _{X} \Phi$ and $d \Phi \neq 0$ on $\partial X_{c}$ since the set of the critical values of $\Phi$ has Lebesgue measure zero by Sard's theorem. Fix such a $c$ in this step. We want to apply Proposition 2.5 to $s u_{\varepsilon, \delta}$ and $v_{\varepsilon, \delta}$, but we do not know whether $v_{\varepsilon, \delta}$ is smooth on $Y_{\varepsilon}$. For this reason, for given $\varepsilon, \delta>0$, we take smooth $F$-valued $(n, q-1)$-forms $\left\{v_{\varepsilon, \delta, k}\right\}_{k=1}^{\infty}$ such that $v_{\varepsilon, \delta, k}$ (respectively, $\left.\bar{\partial} v_{\varepsilon, \delta, k}\right)$ converges to $v_{\varepsilon, \delta}$ (respectively, $\left.\bar{\partial} v_{\varepsilon, \delta}=s u_{\varepsilon, \delta}\right)$ in the $L^{2}$-space $L_{(2)}^{n, \bullet}\left(F^{m+1}\right)_{\varepsilon, \delta}$ (see Lemma 2.4). From now on, we consider only $d(>c)$ satisfying the properties in Proposition 2.5 for countably many differential forms (see Remark 2.6). Then, Proposition 2.5 yields

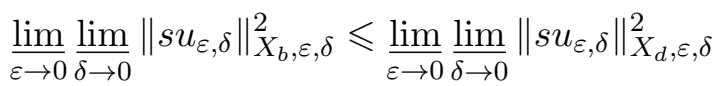

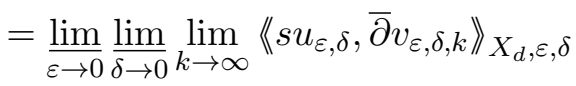

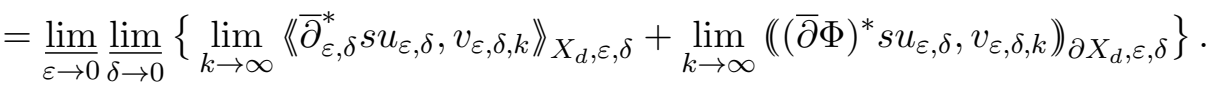

Note that $(\bar{\partial} \Phi)^{*}$ is the adjoint operator of the wedge product $\bar{\partial} \Phi \wedge \bullet$ with respect to $\omega_{\varepsilon, \delta}$. We will show that the first term (respectively, the second term) is zero in Proposition 3.16 (respectively, in Proposition 3.17). For this purpose, we first prove the following proposition. 


\section{INJECTIVITY THEOREMS FOR HIGHER DIRECT IMAGES}

Proposition 3.15. In the above situation, we have

$$
\lim _{\varepsilon \rightarrow 0} \varlimsup_{\delta \rightarrow 0}\left\|D_{\varepsilon, \delta}^{\prime *} u_{\varepsilon, \delta}\right\|_{\varepsilon, \delta}=0 .
$$

Moreover, we have

$$
\lim _{\varepsilon \rightarrow 0} \varlimsup_{\delta \rightarrow 0}\left\|D_{\varepsilon, \delta}^{\prime *} s u_{\varepsilon, \delta}\right\|_{\varepsilon, \delta}=0 \quad \text { and } \quad \lim _{\varepsilon \rightarrow 0} \varlimsup_{\delta \rightarrow 0}\left\|\bar{\partial}_{\varepsilon, \delta}^{*} s u_{\varepsilon, \delta}\right\|_{\varepsilon, \delta}=0 .
$$

Proof. We define $g_{\varepsilon, \delta}$ and $\tilde{g}_{\varepsilon, \delta}$ by

$$
g_{\varepsilon, \delta}:=\left\langle\sqrt{-1} \Theta_{H_{\varepsilon}} \Lambda_{\varepsilon, \delta} u_{\varepsilon, \delta}, u_{\varepsilon, \delta}\right\rangle_{\varepsilon, \delta} \quad \text { and } \quad \tilde{g}_{\varepsilon, \delta}:=\left\langle\sqrt{-1} \Theta_{H_{\varepsilon} h_{\varepsilon}^{m}} \Lambda_{\varepsilon, \delta} u_{\varepsilon, \delta}, u_{\varepsilon, \delta}\right\rangle_{\varepsilon, \delta} .
$$

By definition, we have

$$
\sqrt{-1} \Theta_{H_{\varepsilon} h_{\varepsilon}^{m}} \leqslant \sqrt{-1} \Theta_{H_{\varepsilon}^{m+1}}=(m+1) \sqrt{-1} \Theta_{H_{\varepsilon}} .
$$

Hence, we obtain $\tilde{g}_{\varepsilon, \delta} \leqslant(m+1) g_{\varepsilon, \delta}$. By applying the Bochner-Kodaira-Nakano identity (Proposition 2.3 of the case $\Phi \equiv 0)$ to $u_{\varepsilon, \delta}$ and $s u_{\varepsilon, \delta}$, we obtain

$$
\begin{gathered}
0=\left\|D_{\varepsilon, \delta}^{* *} u_{\varepsilon, \delta}\right\|_{\varepsilon, \delta}^{2}+\int_{Y_{\varepsilon}} g_{\varepsilon, \delta} d V_{\varepsilon, \delta}, \\
\left\|\bar{\partial}_{\varepsilon, \delta}^{*} s u_{\varepsilon, \delta}\right\|_{\varepsilon, \delta}^{2}=\left\|D_{\varepsilon, \delta}^{* *} s u_{\varepsilon, \delta}\right\|_{\varepsilon, \delta}^{2}+\int_{Y_{\varepsilon}}|s|_{h_{\varepsilon}^{m}}^{2} \tilde{g}_{\varepsilon, \delta} d V_{\varepsilon, \delta} .
\end{gathered}
$$

Here we used the equality $\bar{\partial} s u_{\varepsilon, \delta}=s \bar{\partial} u_{\varepsilon, \delta}=0$ and the fact that $u_{\varepsilon, \delta}$ is harmonic with respect to $H_{\varepsilon}$ and $\omega_{\varepsilon, \delta}$. By property (d) and property (B), we have

$$
\sqrt{-1} \Theta_{H_{\varepsilon}}(F)=\sqrt{-1} \Theta_{h_{\varepsilon}}(F)+\sqrt{-1} \partial \bar{\partial} \chi(\Phi) \geqslant-\varepsilon \omega \geqslant-\varepsilon \omega_{\varepsilon, \delta} .
$$

The above inequalities yield

$$
g_{\varepsilon, \delta} \geqslant-\varepsilon q\left|u_{\varepsilon, \delta}\right|_{\varepsilon, \delta}^{2}
$$

Then, we obtain

$$
0 \geqslant \int_{\left\{g_{\varepsilon, \delta} \leqslant 0\right\}} g_{\varepsilon, \delta} d V_{\varepsilon, \delta} \geqslant-\varepsilon q \int_{\left\{g_{\varepsilon, \delta} \leqslant 0\right\}}\left|u_{\varepsilon, \delta}\right|_{\varepsilon, \delta}^{2} d V_{\varepsilon, \delta} \geqslant-\varepsilon q\left\|u_{\varepsilon, \delta}\right\|_{\varepsilon, \delta}^{2} \geqslant-\varepsilon q\|u\|_{H, \omega}^{2}
$$

from inequality (3.6); hence we obtain

$$
\left\|D_{\varepsilon, \delta}^{*} u_{\varepsilon, \delta}\right\|_{\varepsilon, \delta}^{2}+\int_{\left\{g_{\varepsilon, \delta} \geqslant 0\right\}} g_{\varepsilon, \delta} d V_{\varepsilon, \delta} \leqslant-\int_{\left\{g_{\varepsilon, \delta} \leqslant 0\right\}} g_{\varepsilon, \delta} d V_{\varepsilon, \delta} \leqslant \varepsilon q\|u\|_{H, \omega}^{2}
$$

from equality (3.13). This implies that

$$
\lim _{\varepsilon \rightarrow 0} \varlimsup_{\delta \rightarrow 0} \int_{\left\{g_{\varepsilon, \delta} \geqslant 0\right\}} g_{\varepsilon, \delta} d V_{\varepsilon, \delta}=0 \quad \text { and } \quad \lim _{\varepsilon \rightarrow 0} \varlimsup_{\delta \rightarrow 0}\left\|D_{\varepsilon, \delta}^{\prime *} u_{\varepsilon, \delta}\right\|_{\varepsilon, \delta}=0 .
$$

On the other hand, from $\sup _{X}|s|_{h_{\varepsilon}^{m}} \leqslant \sup _{X}|s|_{h^{m}}<\infty$, we have

$$
\begin{aligned}
& \int_{Y_{\varepsilon}}|s|_{h_{\varepsilon}^{m}}^{2} \tilde{g}_{\varepsilon, \delta} d V_{\varepsilon, \delta} \leqslant \int_{\left\{g_{\varepsilon, \delta} \geqslant 0\right\}}|s|_{h_{\varepsilon}^{m}}^{2} \tilde{g}_{\varepsilon, \delta} d V_{\varepsilon, \delta} \leqslant(m+1) \sup _{X}|s|_{h^{m}}^{2} \int_{\left\{g_{\varepsilon, \delta} \geqslant 0\right\}} g_{\varepsilon, \delta} d V_{\varepsilon, \delta}, \\
& \left\|D_{\varepsilon, \delta}^{\prime *} s u_{\varepsilon, \delta}\right\|_{\varepsilon, \delta}=\left\|-* \bar{\partial} * s u_{\varepsilon, \delta}\right\|_{\varepsilon, \delta}=\left\|s D_{\varepsilon, \delta}^{\prime *} u_{\varepsilon, \delta}\right\|_{\varepsilon, \delta} \leqslant \sup _{X}|s|_{h^{m}}\left\|D_{\varepsilon, \delta}^{\prime *} u_{\varepsilon, \delta}\right\|_{\varepsilon, \delta} .
\end{aligned}
$$

These inequalities and equality (3.14) lead to the conclusion.

Proposition 3.16. In the above situation, we have

$$
\lim _{\varepsilon \rightarrow 0} \varlimsup_{\delta \rightarrow 0} \lim _{k \rightarrow \infty}\left\langle\bar{\partial}_{\varepsilon, \delta}^{*} s u_{\varepsilon, \delta}, v_{\varepsilon, \delta, k}\right\rangle_{X_{d}, \varepsilon, \delta}=0 .
$$




\section{S. Matsumura}

Proof. Cauchy-Schwarz's inequality yields

$$
\left\langle\bar{\partial}_{\varepsilon, \delta}^{*} s u_{\varepsilon, \delta}, v_{\varepsilon, \delta, k}\right\rangle_{X_{d}, \varepsilon, \delta} \leqslant\left\|\bar{\partial}_{\varepsilon, \delta}^{*} s u_{\varepsilon, \delta}\right\|_{X_{d}, \varepsilon, \delta}\left\|v_{\varepsilon, \delta, k}\right\|_{X_{d}, \varepsilon, \delta} .
$$

By the construction of $v_{\varepsilon, \delta, k}$, we may assume that

$$
\varlimsup_{\varepsilon \rightarrow 0} \varlimsup_{\delta \rightarrow 0} \lim _{k \rightarrow \infty}\left\|v_{\varepsilon, \delta, k}\right\|_{X_{d}, \varepsilon, \delta}=\varlimsup_{\varepsilon \rightarrow 0} \varlimsup_{\delta \rightarrow 0}\left\|v_{\varepsilon, \delta}\right\|_{X_{d}, \varepsilon, \delta}
$$

is finite (see Corollary 3.14). On the other hand, the $L^{2}$-norm $\left\|\bar{\partial}_{\varepsilon, \delta}^{*} s u_{\varepsilon, \delta}\right\|_{\varepsilon, \delta}$ converges to zero by Proposition 3.15.

We prove the following proposition by using the twisted Bochner-Kodaira-Nakano identity; this completes the proof of Theorem 1.2.

Proposition 3.17. In the above situation, we have

$$
\varliminf_{\varepsilon \rightarrow 0} \lim _{\delta \rightarrow 0} \lim _{k \rightarrow \infty}\left(\left((\bar{\partial} \Phi)^{*} s u_{\varepsilon, \delta}, v_{\varepsilon, \delta, k}\right)\right)_{\partial X_{d}, \varepsilon, \delta}=0
$$

for almost all $d$.

Proof. Cauchy-Schwarz's inequality and Hölder's inequality yield

$$
\begin{aligned}
\left(\left((\bar{\partial} \Phi)^{*} s u_{\varepsilon, \delta}, v_{\varepsilon, \delta, k}\right)\right)_{\partial X_{d}, \varepsilon, \delta} & =\int_{\partial X_{d}}\left\langle(\bar{\partial} \Phi)^{*} s u_{\varepsilon, \delta}, v_{\varepsilon, \delta, k}\right\rangle_{\varepsilon, \delta} d S_{\omega_{\varepsilon, \delta}} \\
& \leqslant \int_{\partial X_{d}}\left|(\bar{\partial} \Phi)^{*} s u_{\varepsilon, \delta}\right|_{\varepsilon, \delta}\left|v_{\varepsilon, \delta, k}\right|_{\varepsilon, \delta} d S_{\omega_{\varepsilon, \delta}} \\
& \leqslant\left(\left((\bar{\partial} \Phi)^{*} s u_{\varepsilon, \delta},(\bar{\partial} \Phi)^{*} s u_{\varepsilon, \delta}\right)\right)_{\partial X_{d}, \varepsilon, \delta}\left(\left(v_{\varepsilon, \delta, k}, v_{\varepsilon, \delta, k}\right)_{\partial X_{d}, \varepsilon, \delta}\right.
\end{aligned}
$$

We first show that the limit of $\left(\left(v_{\varepsilon, \delta, k}, v_{\varepsilon, \delta, k}\right)\right)_{\partial X_{d}, \varepsilon, \delta}$ is finite for almost all $d$. By Fubini's theorem and $d V_{\varepsilon, \delta}=d \Phi \wedge d S_{\omega_{\varepsilon, \delta}}$, we have

$$
\int_{d \in(c-a, c+a)}\left(\left(v_{\varepsilon, \delta, k}, v_{\varepsilon, \delta, k}\right)\right)_{\partial X_{d}, \varepsilon, \delta} d \Phi=\int_{\{c-a<\Phi<c+a\}}\left|v_{\varepsilon, \delta, k}\right|_{\varepsilon, \delta}^{2} d V_{\varepsilon, \delta} .
$$

Furthermore, by Fatou's lemma, we have

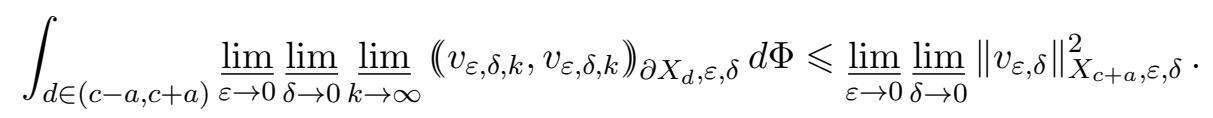

We are assuming that the right-hand side is finite by Corollary 3.14. Therefore, the integrand of the left-hand side is finite for almost all $d \in(c-a, c+a)$.

Finally, we will show that the norm of $(\bar{\partial} \Phi)^{*} s u_{\varepsilon, \delta}$ on $\partial X_{d}$ converges to zero for almost all $d$. By $(\bar{\partial} \Phi)^{*} s u_{\varepsilon, \delta}=s(\bar{\partial} \Phi)^{*} u_{\varepsilon, \delta}$ and $\sup |s|_{h_{\varepsilon}^{m}} \leqslant \sup |s|_{h^{m}}<\infty$, we have

$$
\left(\left((\bar{\partial} \Phi)^{*} s u_{\varepsilon, \delta},(\bar{\partial} \Phi)^{*} s u_{\varepsilon, \delta}\right)\right)_{\partial X_{d}, \varepsilon, \delta} \leqslant \sup _{X}|s|_{h^{m}}^{2}\left(\left((\bar{\partial} \Phi)^{*} u_{\varepsilon, \delta},(\bar{\partial} \Phi)^{*} u_{\varepsilon, \delta}\right)\right)_{\partial X_{d}, \varepsilon, \delta}
$$

Therefore, it is sufficient to show that the norm of $(\bar{\partial} \Phi)^{*} u_{\varepsilon, \delta}$ converges to zero. By applying Proposition 2.5 to $(\bar{\partial} \Phi)^{*} u_{\varepsilon, \delta}$ and $u_{\varepsilon, \delta}$, we obtain

$$
\begin{aligned}
\left\langle\bar{\partial}\left((\bar{\partial} \Phi)^{*} u_{\varepsilon, \delta}\right), u_{\varepsilon, \delta}\right\rangle_{X_{d}, \varepsilon, \delta} & \left.=\left\langle(\bar{\partial} \Phi)^{*} u_{\varepsilon, \delta}, \bar{\partial}_{\varepsilon, \delta}^{*} u_{\varepsilon, \delta}\right\rangle_{X_{d}, \varepsilon, \delta}+\left((\bar{\partial} \Phi)^{*} u_{\varepsilon, \delta},(\bar{\partial} \Phi)^{*} u_{\varepsilon, \delta}\right)\right)_{\partial X_{d}, \varepsilon, \delta} \\
& =\left((\bar{\partial} \Phi)^{*} u_{\varepsilon, \delta},(\bar{\partial} \Phi)^{*} u_{\varepsilon, \delta}\right)_{\partial X_{d}, \varepsilon, \delta} .
\end{aligned}
$$

Here we used the equality $\bar{\partial}_{\varepsilon, \delta}^{*} u_{\varepsilon, \delta}=0$. For the proof, we will compute the left-hand side. Note that we have the equalities $\bar{\partial} u_{\varepsilon, \delta}=0, \partial \Phi \wedge u_{\varepsilon, \delta}=0$, and $\sqrt{-1} \partial \bar{\partial} \Phi \wedge u_{\varepsilon, \delta}=0$ since $u_{\varepsilon, \delta}$ is 


\section{INJECTIVITY THEOREMS FOR HIGHER DIRECT IMAGES}

a $\bar{\partial}$-closed $F$-valued $(n, q)$-form. Therefore, by Lemma 2.1, we obtain

$$
\left\langle\bar{\partial}\left((\bar{\partial} \Phi)^{*} u_{\varepsilon, \delta}\right), u_{\varepsilon, \delta}\right\rangle_{X_{d}, \varepsilon, \delta}=-\left\langle\left\langle\partial \Phi \wedge\left(D_{\varepsilon, \delta}^{\prime *} u_{\varepsilon, \delta}\right), u_{\varepsilon, \delta}\right\rangle_{X_{d}, \varepsilon, \delta}+\left\langle\sqrt{-1} \partial \bar{\partial} \Phi \Lambda u_{\varepsilon, \delta}, u_{\varepsilon, \delta}\right\rangle_{X_{d}, \varepsilon, \delta} \cdot\right.
$$

From Lemma 2.2, inequality (3.6), and Cauchy-Schwarz's inequality, we can estimate the first term of equality (3.16) as follows:

$$
\begin{aligned}
\left|\left\langle\partial \Phi \wedge\left(D_{\varepsilon, \delta}^{\prime *} u_{\varepsilon, \delta}\right), u_{\varepsilon, \delta}\right\rangle_{X_{d}, \varepsilon, \delta}\right| & \leqslant \sup |\partial \Phi|_{\omega_{\varepsilon, \delta}}\left\|D_{\varepsilon, \delta}^{\prime *} u_{\varepsilon, \delta}\right\|_{\varepsilon, \delta}\left\|u_{\varepsilon, \delta}\right\|_{\varepsilon, \delta} \\
& \leqslant \sup |\partial \Phi|_{\omega}\left\|D_{\varepsilon, \delta}^{\prime *} u_{\varepsilon, \delta}\right\|_{\varepsilon, \delta}\|u\|_{H, \omega} .
\end{aligned}
$$

The right-hand side converges to zero by Proposition 3.15 .

To estimate the second term of equality (3.16), by applying Ohsawa-Takegoshi's twisted Bochner-Kodaira-Nakano identity (Proposition 2.3), we obtain

$$
\begin{aligned}
\left\|\sqrt{\eta}(\bar{\partial} \Phi) u_{\varepsilon, \delta}\right\|_{\varepsilon, \delta}^{2} & =\left\|\sqrt{\eta}\left(D_{\varepsilon, \delta}^{\prime *}-(\partial \Phi)^{*}\right) u_{\varepsilon, \delta}\right\|_{\varepsilon, \delta}^{2}+\left\langle\eta \sqrt{-1}\left(\Theta_{H_{\varepsilon}}+\partial \bar{\partial} \Phi\right) \Lambda u_{\varepsilon, \delta}, u_{\varepsilon, \delta}\right\rangle_{\varepsilon, \delta} \\
& \geqslant\left\|\sqrt{\eta}\left(D_{\varepsilon, \delta}^{\prime *}-(\partial \Phi)^{*}\right) u_{\varepsilon, \delta}\right\|_{\varepsilon, \delta}^{2}-\varepsilon C \sup _{X} \eta\left\|u_{\varepsilon, \delta}\right\|_{\varepsilon, \delta}^{2}+\left\langle\sqrt{-1} \partial \bar{\partial} \Phi \Lambda u_{\varepsilon, \delta}, u_{\varepsilon, \delta}\right\rangle_{\varepsilon, \delta},
\end{aligned}
$$

where $\eta$ is the bounded function defined by $\eta:=e^{\Phi}$. The above inequality follows from inequality (3.15). We compute the first term on the right-hand side by using Lemma 2.1 and Cauchy-Schwarz's inequality. It is easy to check that

$$
\begin{aligned}
& \left\|\sqrt{\eta}\left(D_{\varepsilon, \delta}^{\prime *} u_{\varepsilon, \delta}-(\partial \Phi)^{*}\right) u_{\varepsilon, \delta}\right\|_{\varepsilon, \delta}^{2} \\
& \quad \geqslant\left\|\sqrt{\eta} D_{\varepsilon, \delta}^{\prime *} u_{\varepsilon, \delta}\right\|_{\varepsilon, \delta}^{2}-2\left\|\sqrt{\eta} D_{\varepsilon, \delta}^{\prime *} u_{\varepsilon, \delta}\right\|_{\varepsilon, \delta}\left\|\sqrt{\eta}(\partial \Phi)^{*} u_{\varepsilon, \delta}\right\|_{\varepsilon, \delta}+\left\|\sqrt{\eta}(\partial \Phi)^{*} u_{\varepsilon, \delta}\right\|_{\varepsilon, \delta}^{2} \\
& \quad \geqslant-2\left\|\sqrt{\eta} D_{\varepsilon, \delta}^{\prime *} u_{\varepsilon, \delta}\right\|_{\varepsilon, \delta}\left\|\sqrt{\eta}(\partial \Phi)^{*} u_{\varepsilon, \delta}\right\|_{\varepsilon, \delta}+\left\|\sqrt{\eta}(\partial \Phi)^{*} u_{\varepsilon, \delta}\right\|_{\varepsilon, \delta}^{2} .
\end{aligned}
$$

On the other hand, Lemma 2.1 implies $\left|(\partial \Phi)^{*} u_{\varepsilon, \delta}\right|^{2}=\left|(\bar{\partial} \Phi) u_{\varepsilon, \delta}\right|^{2}+\left|(\bar{\partial} \Phi)^{*} u_{\varepsilon, \delta}\right|^{2}$; hence we obtain

$$
\begin{aligned}
\left\|\sqrt{\eta}(\partial \Phi)^{*} u_{\varepsilon, \delta}\right\|_{\varepsilon, \delta}^{2} & =\left\|\sqrt{\eta}(\bar{\partial} \Phi) u_{\varepsilon, \delta}\right\|_{\varepsilon, \delta}^{2}+\left\|\sqrt{\eta}(\bar{\partial} \Phi)^{*} u_{\varepsilon, \delta}\right\|_{\varepsilon, \delta}^{2} \\
& \geqslant\left\|\sqrt{\eta}(\bar{\partial} \Phi) u_{\varepsilon, \delta}\right\|_{\varepsilon, \delta}^{2} .
\end{aligned}
$$

From these inequalities, we have

$$
\varepsilon C \sup _{X} \eta\left\|u_{\varepsilon, \delta}\right\|_{\varepsilon, \delta}^{2}+2\left\|\sqrt{\eta} D_{\varepsilon, \delta}^{\prime *} u_{\varepsilon, \delta}\right\|_{\varepsilon, \delta}^{2}\left\|\sqrt{\eta}(\partial \Phi)^{*} u_{\varepsilon, \delta}\right\|_{\varepsilon, \delta}^{2} \geqslant\left\langle\sqrt{-1} \partial \bar{\partial} \Phi \Lambda u_{\varepsilon, \delta}, u_{\varepsilon, \delta}\right\rangle_{\varepsilon, \delta} \geqslant 0 .
$$

The norm $\left\|\sqrt{\eta} D_{\varepsilon, \delta}^{\prime *} u_{\varepsilon, \delta}\right\|_{\varepsilon, \delta}^{2}$ converges to zero by Proposition 3.15, and the norm $\left\|\sqrt{\eta}(\partial \Phi)^{*} u_{\varepsilon, \delta}\right\|_{\varepsilon, \delta}^{2}$ is uniformly bounded by Lemma 2.2. This completes the proof.

\subsection{Proof of Theorem 1.3}

In this subsection, we explain how to adapt the proof of Theorem 1.2 to deduce Theorem 1.3.

Proof of Theorem 1.3. The proof is a slight variation of that of Theorem 1.2. We give only the several differences with the proof of Theorem 1.2.

In Step 1, by applying Theorem 2.9 to $\gamma=b \sqrt{-1} \Theta_{h_{M}}(M)$, we take a family of singular metrics $\left\{h_{\varepsilon}\right\}_{1 \gg \varepsilon>0}$ on $F$ with the following properties:

(a) The metric $h_{\varepsilon}$ is smooth on $X \backslash Z_{\varepsilon}$ for some proper subvariety $Z_{\varepsilon}$.

(b) The inequalities $h_{\varepsilon^{\prime \prime}} \leqslant h_{\varepsilon^{\prime}} \leqslant h$ hold on $X$ for any $0<\varepsilon^{\prime}<\varepsilon^{\prime \prime}$.

(c) We have $\mathcal{I}(h)=\mathcal{I}\left(h_{\varepsilon}\right)$ on $X$.

(e) We have $\sqrt{-1} \Theta_{h_{\varepsilon}}(F) \geqslant b \sqrt{-1} \Theta_{h_{M}}(M)-\varepsilon \omega$ on $X$. 


\section{S. Matsumura}

Note that property (e) is obtained from the assumption $\sqrt{-1} \Theta_{h}(F) \geqslant b \sqrt{-1} \Theta_{h_{M}}(M)$. We can see that property (e) is stronger than property $(\mathrm{d})$ in the proof of Theorem 1.2. Indeed, by the assumption $\sqrt{-1} \Theta_{h_{M}}(M) \geqslant 0$, we obtain property (d)

(d) We have $\sqrt{-1} \Theta_{h_{\varepsilon}}(F) \geqslant-\varepsilon \omega$ on $X$.

By property (d), we can see that the same argument as in Step 2 works.

In Step 3, by considering the norm $\left\|s u_{\varepsilon, \delta}\right\|_{\varepsilon, \delta}:=\left\|s u_{\varepsilon, \delta}\right\|_{H_{\varepsilon} h_{M}, \omega_{\varepsilon, \delta}}$ instead of $\left\|s u_{\varepsilon, \delta}\right\|_{H_{\varepsilon} h_{\varepsilon}{ }^{m}, \omega_{\varepsilon, \delta}}$, we can easily prove the same conclusion as in Proposition 3.7.

In Step 4, we can obtain $v_{\varepsilon} \in L_{(2)}^{n, q-1}(F \otimes M)_{\varepsilon, \delta}$ with the properties of Corollary 3.14 since we do not use the line bundle $M$ when we prove Proposition 3.9.

In Step 5, we need to prove the following proposition (see Proposition 3.15). Recall that Propositions 3.16 and 3.17 finish the proof of Theorem 1.2 and that they are obtained from Proposition 3.15 .

Proposition 3.18 (cf. Proposition 3.15). We have

$$
\lim _{\varepsilon \rightarrow 0} \varlimsup_{\delta \rightarrow 0}\left\|D_{\varepsilon, \delta}^{*} u_{\varepsilon, \delta}\right\|_{\varepsilon, \delta}=0 .
$$

Moreover, we have

$$
\lim _{\varepsilon \rightarrow 0} \varlimsup_{\delta \rightarrow 0}\left\|D_{\varepsilon, \delta}^{\prime *} s u_{\varepsilon, \delta}\right\|_{\varepsilon, \delta}=0 \quad \text { and } \quad \lim _{\varepsilon \rightarrow 0} \varlimsup_{\delta \rightarrow 0}\left\|\bar{\partial}_{\varepsilon, \delta}^{*} s u_{\varepsilon, \delta}\right\|_{\varepsilon, \delta}=0 .
$$

Proof. By applying the Bochner-Kodaira-Nakano identity to $u_{\varepsilon, \delta}$ and $s u_{\varepsilon, \delta}$, we obtain the following equalities:

$$
\begin{gathered}
0=\left\|D_{\varepsilon, \delta}^{\prime *} u_{\varepsilon, \delta}\right\|_{\varepsilon, \delta}^{2}+\int_{Y_{\varepsilon}} g_{\varepsilon, \delta} d V_{\varepsilon, \delta} \\
\left\|\bar{\partial}_{\varepsilon, \delta}^{*} s u_{\varepsilon, \delta}\right\|_{\varepsilon, \delta}^{2}=\left\|D_{\varepsilon, \delta}^{\prime *} s u_{\varepsilon, \delta}\right\|_{\varepsilon, \delta}^{2}+\int_{Y_{\varepsilon}}|s|_{h_{M}}^{2}\left(f_{\varepsilon, \delta}+g_{\varepsilon, \delta}\right) d V_{\varepsilon, \delta},
\end{gathered}
$$

where the integrands $g_{\varepsilon, \delta}$ and $f_{\varepsilon, \delta}$ are the functions defined by

$$
\begin{aligned}
g_{\varepsilon, \delta} & :=\left\langle\sqrt{-1} \Theta_{H_{\varepsilon}}(F) \Lambda_{\varepsilon, \delta} u_{\varepsilon, \delta}, u_{\varepsilon, \delta}\right\rangle_{\varepsilon, \delta}, \\
f_{\varepsilon, \delta} & :=\left\langle\sqrt{-1} \Theta_{h_{M}}(M) \Lambda_{\varepsilon, \delta} u_{\varepsilon, \delta}, u_{\varepsilon, \delta}\right\rangle_{\varepsilon, \delta} .
\end{aligned}
$$

From property (d), we obtain

$$
g_{\varepsilon, \delta} \geqslant-\varepsilon q\left|u_{\varepsilon, \delta}\right|_{\varepsilon, \delta}^{2}
$$

By the same argument as in the proof of Proposition 3.15, we can see that

$$
\lim _{\varepsilon \rightarrow 0} \varlimsup_{\delta \rightarrow 0}\left\|D_{\varepsilon, \delta}^{\prime *} u_{\varepsilon, \delta}\right\|_{\varepsilon, \delta}^{2}=0 \quad \text { and } \quad \lim _{\varepsilon \rightarrow 0} \varlimsup_{\delta \rightarrow 0} \int_{\left\{g_{\varepsilon, \delta} \geqslant 0\right\}} g_{\varepsilon, \delta} d V_{\varepsilon, \delta}=0 .
$$

Therefore, from $\sup _{X}|s|_{h_{M}}<\infty$, we can see that $\left\|D_{\varepsilon, \delta}^{\prime *} s u_{\varepsilon, \delta}\right\|_{\varepsilon, \delta}=\left\|s D_{\varepsilon, \delta}^{\prime *} u_{\varepsilon, \delta}\right\|_{\varepsilon, \delta}$ converges to zero. On the other hand, from property (e), we can easily check

$$
f_{\varepsilon, \delta} \leqslant \frac{1}{b}\left(g_{\varepsilon, \delta}+\varepsilon q\left|u_{\varepsilon, \delta}\right|_{\varepsilon, \delta}^{2}\right) \text {. }
$$


This implies that

$$
\begin{aligned}
\int_{Y_{\varepsilon}}|s|_{h_{M}}^{2}\left(f_{\varepsilon, \delta}+g_{\varepsilon, \delta}\right) d V_{\varepsilon, \delta} & \leqslant \int_{Y_{\varepsilon}}|s|_{h_{M}}^{2}\left\{\left(1+\frac{1}{b}\right) g_{\varepsilon, \delta}+\frac{\varepsilon q}{b}\left|u_{\varepsilon, \delta}\right|_{\varepsilon, \delta}^{2}\right\} d V_{\varepsilon, \delta} \\
& \leqslant \int_{\left\{g_{\varepsilon, \delta} \geqslant 0\right\}}|s|_{h_{M}}^{2}\left\{\left(1+\frac{1}{b}\right) g_{\varepsilon, \delta}+\frac{\varepsilon q}{b}\left|u_{\varepsilon, \delta}\right|_{\varepsilon, \delta}^{2}\right\} d V_{\varepsilon, \delta} \\
& \leqslant \sup _{X}|s|_{h_{M}}^{2}\left(1+\frac{1}{b}\right)\left\{\int_{\left\{g_{\varepsilon, \delta} \geqslant 0\right\}} g_{\varepsilon, \delta} d V_{\varepsilon, \delta}+\frac{\varepsilon q}{b}\|u\|_{H, \omega}^{2}\right\} .
\end{aligned}
$$

This completes the proof.

By this proposition, we can prove the same conclusion as in Propositions 3.16 and 3.17. Therefore, we obtain the conclusion of Theorem 1.3.

\section{Applications}

\subsection{Proof of Corollary 1.5}

Proof of Corollary 1.5. We apply Theorem 1.2 in the case $m=0$ to a holomorphic function $s$. For an open set $B \subset \Delta$ and a holomorphic function $s$ on $\pi^{-1}(B)$, the multiplication map

$$
\Phi_{s}: R^{q} \pi_{*}\left(K_{X} \otimes F \otimes \mathcal{I}(h)\right) \stackrel{\otimes s}{\longrightarrow} R^{q} \pi_{*}\left(K_{X} \otimes F \otimes \mathcal{I}(h)\right)
$$

is injective for every $q$. This implies that $R^{q} \pi_{*}\left(K_{X} \otimes F \otimes \mathcal{I}(h)\right)$ is torsion-free.

\subsection{Proof of Theorem 1.7}

We first recall the definition of the numerical Kodaira dimension of a singular Hermitian line bundle on a projective varieties (see [Cao14] for Kähler manifolds).

Definition 4.1 (Numerical Kodaira dimension, [Cao14]). Let $(F, h)$ be a singular Hermitian line bundle on a smooth projective variety $X$ such that $\sqrt{-1} \Theta_{h}(F) \geqslant 0$. Then, the numerical Kodaira dimension $\operatorname{nd}(F, h)$ of $(F, h)$ is defined to be $\operatorname{nd}(F, h):=-\infty$ if $h \equiv \infty$ and otherwise

$$
\operatorname{nd}(F, h):=\operatorname{dim} X-\varliminf_{\varepsilon \rightarrow 0} \frac{\log \operatorname{vol}_{X}\left(A^{\varepsilon} \otimes F, h\right)}{\log \varepsilon},
$$

where $\operatorname{vol}_{X}\left(A^{\varepsilon} \otimes F, h\right)$ is defined by

$$
\operatorname{vol}_{X}\left(A^{\varepsilon} \otimes F, h\right):=\varlimsup_{m \rightarrow \infty} \frac{h^{0}\left(X, A^{m \varepsilon} \otimes F^{m} \otimes \mathcal{I}\left(h^{m}\right)\right)}{m^{\operatorname{dim} X}} .
$$

By combining Cao's result [Cao14] with the strong openness theorem [GZ15] (see also [Mat14]), we have the following vanishing theorem. (See [Hie14, Lem17] for other proofs of the strong openness theorem.)

Theorem 4.2 ([Cao14, Theorem 1.3], [GZ15, Theorem 1.1]). Let $(F, h)$ be a singular Hermitian line bundle on a compact Kähler manifold $X$ such that $\sqrt{-1} \Theta_{h}(F) \geqslant 0$. Then, we have

$$
H^{q}\left(X, K_{X} \otimes F \otimes \mathcal{I}(h)\right)=0 \quad \text { for every } q>\operatorname{dim} X-\operatorname{nd}(F, h) .
$$

Let us prove Proposition 1.6.

Proof of Proposition 1.6. For a positive integer $m$, we define $Q_{m}$ by

$$
Q_{m}:=\left\{t \in \Delta\left|\mathcal{I}\left(\left.h\right|_{X_{t}} ^{m}\right)=\mathcal{I}\left(h^{m}\right)\right|_{X_{t}}\right\} .
$$




\section{S. Matsumura}

Note that we have $\left.\mathcal{I}\left(\left.h\right|_{X_{t}} ^{m}\right) \subset \mathcal{I}\left(h^{m}\right)\right|_{X_{t}}$ by the Ohsawa-Takegoshi $L^{2}$-extension theorem. By Fubini's theorem, we can see that $\Delta \backslash Q_{m}$ has Lebesgue measure zero. We set $Q:=\cap_{m=1}^{\infty} Q_{m}$. Then $\Delta \backslash Q$ also has Lebesgue measure zero. Let $A$ be a relatively ample line bundle on $X$. By the definition of the numerical dimension, it is sufficient to show that

$$
h^{0}\left(X_{t}, \mathcal{O}_{X_{t}}\left(A^{m \varepsilon} \otimes F^{m}\right) \otimes \mathcal{I}\left(\left.h\right|_{X_{t}} ^{m}\right)\right) \geqslant h^{0}\left(X_{t_{0}}, \mathcal{O}_{X_{t_{0}}}\left(A^{m \varepsilon} \otimes F^{m}\right) \otimes \mathcal{I}\left(\left.h\right|_{X_{t_{0}}} ^{m}\right)\right)
$$

for every $t \in Q$ and $m \gg 0$.

For the canonical bundle $K_{X}$ on $X$, we have

$$
A^{m \varepsilon} \otimes F^{m}=K_{X} \otimes\left(A^{m \varepsilon} \otimes K_{X}^{-1}\right) \otimes F^{m} .
$$

By replacing $\Delta$ with a smaller disk, we may assume that $A^{m \varepsilon} \otimes K_{X}^{-1}$ admits a smooth (Hermitian) metric $g_{m}$ with positive curvature for a sufficiently large $m \gg 0$. Furthermore, we can extend a basis $\left\{s_{i}\right\}_{i \in I}$ in $H^{0}\left(X_{t_{0}}, \mathcal{O}_{X_{t_{0}}}\left(A^{m \varepsilon} \otimes F^{m}\right) \otimes \mathcal{I}\left(\left.h\right|_{X_{t_{0}}} ^{m}\right)\right.$ to sections $\left\{\tilde{s}_{i}\right\}$ in $H^{0}\left(X, \mathcal{O}_{X}\left(A^{m \varepsilon} \otimes F^{m}\right) \otimes\right.$ $\left.\mathcal{I}\left(h^{m}\right)\right)$, by applying the Ohsawa-Takegoshi $L^{2}$ extension theorem to $\left(A^{m \varepsilon} \otimes K_{X}^{-1} \otimes F^{m}, g_{m} h^{m}\right)$ (see [OT87] and [Man93]).

We can easily see that, for every $t$ in some open neighborhood $B$ of $t_{0}$, the elements of $\left\{\left.\tilde{s}_{i}\right|_{X_{t}}\right\}_{i \in I}$ are linearly independent in $H^{0}\left(X_{t},\left.\mathcal{O}_{X_{t}}\left(A^{m \varepsilon} \otimes F^{m}\right) \otimes \mathcal{I}\left(h^{m}\right)\right|_{X_{t}}\right)$. Indeed, if there exist a point $t$ converging to $t_{0}$ and $a_{t, i} \in \mathbb{C}$ such that $\left.\sum_{i \in I} a_{t, i} \tilde{s}_{i}\right|_{X_{t}}=0$, then we may assume that $a_{t, i}$ converges to some $a_{i}$ as $t \rightarrow t_{0}$. As $t \rightarrow t_{0}$, we obtain $\left.\sum_{i \in I} a_{i} \tilde{s}_{i}\right|_{X_{t_{0}}}=0$ from $\left.\sum_{i \in I} a_{t, i} \tilde{s}_{i}\right|_{X_{t}}=0$. Therefore, the elements of $\left\{\left.\tilde{s}_{i}\right|_{X_{t}}\right\}_{i \in I}$ are linearly independent. If $t \in Q$, the restriction $\left.\tilde{s}_{i}\right|_{X_{t}}$ to $X_{t}$ is a section in $H^{0}\left(X_{t}, \mathcal{O}_{X_{t}}\left(A^{m \varepsilon} \otimes F^{m}\right) \otimes \mathcal{I}\left(\left.h\right|_{X_{t}} ^{m}\right)\right)$. This completes the proof.

Remark 4.3. By the proof of Proposition 1.6, we can assume $\mathcal{I}\left(\left.h\right|_{X_{t}} ^{m}\right)=\left.\mathcal{I}\left(h^{m}\right)\right|_{X_{t}}$ for every $t \in Q$.

Proof of Theorem 1.7. We take a point $t_{0}$ with

$$
\operatorname{nd}\left(\left.F\right|_{X_{t_{0}}},\left.h\right|_{X_{t_{0}}}\right)=\max _{\substack{\text { is smooth } \\ \text { at } t \in \Delta}} \operatorname{nd}\left(\left.F\right|_{X_{t}},\left.h\right|_{X_{t}}\right) .
$$

By Proposition 1.6 and Remark 4.3, we can take a dense subset $Q$ in some neighborhood $B$ of $t_{0}$ such that $\operatorname{nd}\left(\left.F\right|_{X_{t}},\left.h\right|_{X_{t}}\right) \geqslant \operatorname{nd}\left(\left.F\right|_{X_{t_{0}}},\left.h\right|_{X_{t_{0}}}\right)$ and $\mathcal{I}\left(\left.h\right|_{X_{t}} ^{m}\right)=\left.\mathcal{I}\left(h^{m}\right)\right|_{X_{t}}$ for every $t \in Q$. Therefore, from Theorem 4.2, we obtain

$$
H^{q}\left(X_{t}, \mathcal{O}_{X_{t}}\left(K_{X} \otimes F\right) \otimes \mathcal{I}(h)\right)=H^{q}\left(X_{t}, \mathcal{O}_{X_{t}}\left(K_{X} \otimes F\right) \otimes \mathcal{I}\left(\left.h\right|_{X_{t}}\right)\right)=0
$$

for $q>f-\operatorname{nd}\left(\left.F\right|_{X_{t_{0}}},\left.h\right|_{X_{t_{0}}}\right) \geqslant f-\operatorname{nd}\left(\left.F\right|_{X_{t}},\left.h\right|_{X_{t}}\right)$ and for every $t \in Q \cap \Delta^{\prime}$. Here $\Delta^{\prime}$ is the Zariski open set in $\Delta$ defined by

$$
\Delta^{\prime}:=\left\{t \in \Delta \mid \pi \text { is smooth at } t \text { and } R^{q} \pi_{*}\left(K_{X} \otimes F \otimes \mathcal{I}(h)\right) \text { is locally free at } t\right\} .
$$

By the flat base change theorem, we obtain $R^{q} \pi_{*}\left(K_{X} \otimes F \otimes \mathcal{I}(h)\right)_{t}=0$ for every $t \in Q \cap \Delta^{\prime}$. This implies that $R^{q} \pi_{*}\left(K_{X} \otimes F \otimes \mathcal{I}(h)\right)_{t}=0$ on $\Delta^{\prime}$. We obtain the conclusion since $R^{q} \pi_{*}\left(K_{X} \otimes F \otimes \mathcal{I}(h)\right)$ is torsion-free.

\subsection{Proof of Corollary 1.9}

Proof of Corollary 1.9. Let $s$ be a holomorphic function on $X$ with $X_{0}=s^{-1}(0)$. By Theorem 1.2, we can conclude that

$$
H^{1}\left(X, K_{X} \otimes F \otimes \mathcal{I}(h)\right) \stackrel{\otimes s}{\longrightarrow} H^{1}\left(X, K_{X} \otimes F \otimes \mathcal{I}(h)\right)
$$




\section{INJECTIVITY THEOREMS FOR HIGHER DIRECT IMAGES}

is injective for a sufficiently small $\Delta$. On the other hand, since $X_{0}$ is a subvariety of codimension 1 and $R^{q} \pi_{*}\left(K_{X} \otimes F \otimes \mathcal{I}(h)\right)$ is torsion-free, the following sequence is exact:

$$
0 \rightarrow \mathcal{O}_{X}\left(K_{X} \otimes F \otimes \mathcal{I}(h)\right) \otimes \mathcal{I}_{X_{0}} \rightarrow \mathcal{O}_{X}\left(K_{X} \otimes F \otimes \mathcal{I}(h)\right) \rightarrow \mathcal{O}_{X_{0}}\left(K_{X} \otimes F \otimes \mathcal{I}(h)\right) \rightarrow 0 .
$$

The induced long exact sequence implies that, for every section $t$ in $H^{0}\left(X_{0}, \mathcal{O}_{X_{0}}\left(K_{X} \otimes F \otimes \mathcal{I}(h)\right)\right)$, there exists a section $T$ in $H^{0}\left(X, \mathcal{O}_{X}\left(K_{X} \otimes F \otimes \mathcal{I}(h)\right)\right)$ such that $\left.T\right|_{X_{0}}=t$. Furthermore, we have the following commutative diagram:

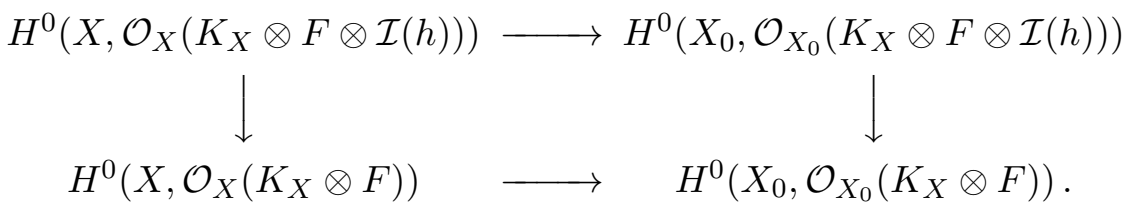

Therefore, we can extend a section in $H^{0}\left(X, \mathcal{O}_{X}\left(K_{X} \otimes F\right)\right)$ that comes from $H^{0}\left(X_{0}, \mathcal{O}_{X_{0}}\left(K_{X} \otimes\right.\right.$ $F \otimes \mathcal{I}(h)))$ to $X$.

\section{ACKNOWLEDGEMEnTS}

The author wishes to express his gratitude to Professor Osamu Fujino for giving several remarks on the proof of Theorem 1.2 for the case $m=0$ and for suggesting him to consider Theorem 1.7. He is sincerely grateful to the referee for carefully reading this paper including technical proofs and for giving helpful comments, which eliminated an unnecessary assumption in Proposition 2.19.

\section{REFERENCES}

Amb03 F. Ambro, Quasi-log varieties, Proc. Steklov Inst. Math. 240 (2003), no. 1, 214-233.

Amb14_, An injectivity theorem, Compos. Math. 150 (2014), no. 6, 999-1023; doi:10.1112/ S0010437X13007768.

AV61 A. Andreotti and E. Vesentini, Sopra un teorema di Kodaira, Ann. Scuola Norm. Sup. Pisa Cl. Sci. (3) 15 (1961), no. 4, 283-309.

AV65_, Carleman estimates for the Laplace-Beltrami equation on complex manifolds, Publ. Math. Inst. Hautes Études Sci. 25 (1965), 81-130; doi:10.1007/BF02684398.

Cao14 J. Cao, Numerical dimension and a Kawamata-Viehweg-Nadel-type vanishing theorem on compact Kähler manifolds, Compos. Math. 150 (2014), no. 11, 1869-1902; doi:10.1112/ S0010437X14007398.

CDM17 J. Cao, J.-P. Demailly, and S. Matsumura, A general extension theorem for cohomology classes on non reduced analytic subspaces, Sci. China Math. 60 (2017), no. 6, 949-962; doi:10.1007/ s11425-017-9066-0.

CP20 J. Cao and M. Păun, On extension of pluricanonical forms defined on the central fiber of a kähler family, 2020, arXiv:2012.05063.

Dem82 J.-P. Demailly, Estimations $L^{2}$ pour l'opérateur $\bar{\partial}$ d'un fibré vectoriel holomorphe semi-positif au-dessus d'une variété kählérienne complète, Ann. Sci. École Norm. Sup. (4) 15 (1982), no. 3, 457-511; doi:10.24033/asens. 1434.

DF83 H. Donnelly and C. Fefferman, $L^{2}$-cohomology and index theorem for the Bergman metric, Ann. of Math. 118 (1983), no. 3, 593-618; doi:10.2307/2006983.

DHP13 J.-P. Demailly, C.D. Hacon, and M. Păun, Extension theorems, non-vanishing and the existence of good minimal models, Acta Math. 210 (2013), no. 2, 203-259; doi:10.1007/ s11511-013-0094-x. 


\section{S. Matsumura}

DPS01 J.-P. Demailly, T. Peternell, and M. Schneider, Pseudo-effective line bundles on compact Kähler manifolds, Internat. J. Math. 12 (2001), no. 6, 689-741; doi:10.1142/S0129167X01000861.

DX84 H. Donnelly and F. Xavier, On the differential form spectrum of negatively curved Riemannian manifolds, Amer. J. Math. 106 (1984), no. 1, 169-185; doi:10.2307/2374434.

Eno93 I. Enoki, Kawamata-Viehweg vanishing theorem for compact Kähler manifolds, Einstein Metrics and Yang-Mills Connections (Sanda, 1990), Lecture Notes in Pure and Appl. Math., vol. 145 (Dekker, New York, 1993), 59-68.

EV92 H. Esnault and E. Viehweg, Lectures on vanishing theorems, DMV Seminar, vol. 20 (Birkhäuser Verlag, Basel, 1992); doi:10.1007/978-3-0348-8600-0.

FG14 O. Fujino and Y. Gongyo, Log pluricanonical representations and the abundance conjecture, Compos. Math. 150 (2014), no. 4, 593-620; doi:10.1112/S0010437X13007495.

FK72 G. B. Folland and J. J. Kohn, The Neumann problem for the Cauchy-Riemann complex, Ann. of Math. Stud., vol. 75 (Princeton Univ. Press, Princeton, NJ; Univ. of Tokyo Press, Tokyo; 1972).

FM21 O. Fujino and S. Matsumura, Injectivity theorem for pseudo-effective line bundles and its applications, Trans. Amer. Math. Soc. Ser. B 8 (2021), 849-884; doi:10.1090/btran/86.

Fuj09 O. Fujino, On injectivity, vanishing and torsion-free theorems for algebraic varieties, Proc. Japan Acad. Ser. A Math. Sci. 85 (2009), no. 8, 95-100; doi:10.3792/pjaa.85.95.

Fuj11_, Fundamental theorems for the log minimal model program, Publ. Res. Inst. Math. Sci. 47 (2011), no. 3, 727-789; doi:10.2977/PRIMS/50.

Fuj12 A transcendental approach to Kollár's injectivity theorem, Osaka J. Math. 49 (2012), no. $3,833-852$.

Fuj13 - A transcendental approach to Kollár's injectivity theorem II, J. reine angew. Math. 681 (2013), 149-174; doi:10.1515/crelle-2012-0036.

Fuj14_, Fundamental theorems for semi log canonical pairs, Algebr. Geom. 1 (2014), no. 2, 194-228; doi:10.14231/AG-2014-011.

Fuj17_ Fundamental theorems for semi log canonical pairs, in Higher Dimensional Algebraic Geometry - in Honour of Professor Yujiro Kawamata's Sixtieth Birthday, Adv. Stud. Pure Math., vol. 74 (Math. Soc. Japan, Tokyo, 2017), 131-157; doi:10.2969/aspm/07410131.

GM17 Y. Gongyo and S. Matsumura, Versions of injectivity and extension theorems, Ann. Sci. Éc. Norm. Supér. (4) 50 (2017), no. 2, 479-502; doi:10.24033/asens. 2325.

GR70 H. Grauert and O. Riemenschneider, Verschwindungssätze für analytische Kohomologiegruppen auf komplexen Räumen, Invent. Math. 11 (1970), 263-292; doi:10.1007/BF01403182.

GR65 R. C. Gunning and H. Rossi, Analytic functions of several complex variables (Prentice-Hall, Inc., Englewood Cliffs, NJ, 1965).

GZ15 Q. Guan and X. Zhou, A proof of Demailly's strong openness conjecture, Ann. of Math. 182 (2015), no. 2, 605-616; doi:10.4007/annals.2015.182.2.5.

Hie14 P. H. Hiep, The weighted log canonical threshold, C. R. Math. Acad. Sci. Paris 352 (2014), no. 4, 283-288; doi:10.1016/j.crma.2014.02.010.

Hör65 L. Hörmander, $L^{2}$ estimates and existence theorems for the $\bar{\partial}$ operator, Acta Math. 113 (1965), 89-152; doi:10.1007/BF02391775.

Kaw82 Y. Kawamata, A generalization of Kodaira-Ramanujam's vanishing theorem, Math. Ann. 261 (1982), no. 1, 43-46; doi:10.1007/BF01456407.

Kod53 K. Kodaira, On a differential-geometric method in the theory of analytic stacks, Proc. Nat. Acad. Sci. USA 39 (1953), 1268-1273; doi:10.1073/pnas.39.12.1268.

Kol86a J. Kollár, Higher direct images of dualizing sheaves. I, Ann. of Math. 123 (1986), no. 1, 11-42; doi: $10.2307 / 1971351$.

Kol86b - Higher direct images of dualizing sheaves. II, Ann. of Math. 124 (1986), no. 1, 171-202; doi:10.2307/1971390. 


\section{INJECTIVITY THEOREMS FOR HIGHER DIRECT IMAGES}

Lem17 L. Lempert, Modules of square integrable holomorphic germs, Analysis Meets Geometry, Trends Math. (Birkhäuser/Springer, Cham, 2017), 311-333; doi:10.1007/978-3-319-52471-9_19.

Lev83 M. Levine, Pluri-canonical divisors on Kähler manifolds, Invent. Math. 74 (1983), no. 2, 293-303; doi:10.1007/BF01394318.

LRW19 K. Liu, S. Rao, and X. Wan, Geometry of logarithmic forms and deformations of complex structures, J. Algebraic Geom. 28 (2019), no. 4, 773-815; doi:10.1090/jag/723.

Man93 L. Manivel, Un théorème de prolongement $L^{2}$ de sections holomorphes d'un fibré hermitien, Math. Z. 212 (1993), no. 1, 107-122; doi:10.1007/BF02571643.

Mat14 S. Matsumura, A Nadel vanishing theorem via injectivity theorems, Math. Ann. 359 (2014), no. 3-4, 785-802; doi:10.1007/s00208-014-1018-6.

Mat15a , Injectivity theorems with multiplier ideal sheaves and their applications, in Complex Analysis and Geometry, Springer Proc. Math. Stat., vol. 144 (Springer, Tokyo, 2015), 241-255; doi:10.1007/978-4-431-55744-9_18.

Mat15b _ A Nadel vanishing theorem for metrics with minimal singularities on big line bundles, Adv. Math. 280 (2015), 188-207; doi:10.1016/j.aim.2015.03.019.

Mat18a _ An injectivity theorem with multiplier ideal sheaves of singular metrics with transcendental singularities, J. Algebraic Geom. 27 (2018), no. 2, 305-337; doi:10.1090/jag/687.

Mat18b _ Variation of numerical dimension of singular hermitian line bundles, in Geometric Complex Analysis, Springer Proc. Math. Stat., vol. 246 (Springer, Singapore, 2018), 247-255; doi:10.1007/978-981-13-1672-2_19.

Mat19 A A transcendental approach to injectivity theorem for log canonical pairs, Ann. Sc. Norm. Super. Pisa Cl. Sci. (5) 19 (2019), no. 1, 311-334; doi:10.2422/2036-2145.201702_018.

Nad90 A. M. Nadel, Multiplier ideal sheaves and Kähler-Einstein metrics of positive scalar curvature, Ann. of Math. 132 (1990), no. 3, 549-596; doi:10.2307/1971429.

Ohs95 T. Ohsawa, On the extension of $L^{2}$ holomorphic functions. III. Negligible weights, Math. Z. 219 (1995), no. 2, 215-225; doi:10.1007/BF02572360.

Ohs05_, On a curvature condition that implies a cohomology injectivity theorem of Kollár-Skoda type, Publ. Res. Inst. Math. Sci. 41 (2005), no. 3, 565-577; doi:10.2977/PRIMS/1145475223.

OT87 T. Ohsawa and K. Takegoshi, On the extension of $L^{2}$ holomorphic functions, Math. Z. 195 (1987), no. 2, 197-204; doi:10.1007/BF01166457.

Pău07 M. Păun, Siu's invariance of plurigenera: a one-tower proof, J. Differential Geom. 76 (2007), no. 3, 485-493; doi:10.4310/jdg/1180135695.

Pri71 D. Prill, The divisor class groups of some rings of holomorphic functions, Math. Z. 121 (1971), 58-80; doi:10.1007/BF01110367.

Siu98 Y.-T. Siu, Invariance of plurigenera, Invent. Math. 134 (1998), no. 3, 661-673; doi:10.1007/ s002220050276.

Siu02_, Extension of twisted pluricanonical sections with plurisubharmonic weight and invariance of semipositively twisted plurigenera for manifolds not necessarily of general type, Complex Geometry (Göttingen, 2000) (Springer, Berlin, 2002), 223-277; doi:10.1007/ 978-3-642-56202-0_15.

SZ21 J. Shentu and C. Zhao, $L^{2}$-extension of adjoint bundles and Kollár's conjecture, 2021, arXiv: 2106.13407.

Tak07 S. Takayama, On the invariance and the lower semi-continuity of plurigenera of algebraic varieties, J. Algebraic Geom. 16 (2007), no. 1, 1-18; doi:10.1090/S1056-3911-06-00455-3.

Tak95 K. Takegoshi, Higher direct images of canonical sheaves tensorized with semi-positive vector bundles by proper Kähler morphisms, Math. Ann. 303 (1995), no. 3, 389-416; doi:10.1007/ BF01460997.

Tak97 _ On cohomology groups of nef line bundles tensorized with multiplier ideal sheaves on compact Kähler manifolds, Osaka J. Math. 34 (1997), no. 4, 783-802. 


\section{S. Matsumura}

Tan71 S. G. Tankeev, $n$-dimensional canonically polarized varieties, and varieties of basic type, Math. USSR-Izv. 5 (1971), no. 1, 29-43.

Ves67 E. Vesentini, Lectures on Levi convexity of complex manifolds and cohomology vanishing theorems, Tata Inst. Fund. Res. Stud. Math., vol. 39 (Tata Inst. Fund. Res., Bombay, 1967).

Vie82 E. Viehweg, Vanishing theorems, J. reine angew. Math. 335 (1982), 1-8; doi:10.1515/crll. 1982.335.1.

ZZ19 X. Zhou and L. Zhu, Extension of cohomology classes and holomorphic sections defined on subvarieties, 2019, arXiv:1909.08822.

Shin-ichi Matsumura mshinichi-math@tohoku.ac.jp mshinichi0@gmail.com

Mathematical Institute, Tohoku University, 6-3, Aramaki Aza-Aoba, Aoba-ku, Sendai 980-8578, Japan 